\title{
A Lei de Weyl para o Laplaciano
}

Rafael Moreira Neves

DisSERTAÇÃo APRESENTADA

$\mathrm{AO}$

Instituto DE MAtemática E Estatística

DA

Universidade de São Paulo

PARA

OBTENÇÃO DO TÍTULO

$\mathrm{DE}$

Mestre em CiênCIAS

Programa: Matemática Aplicada

Orientador: Prof. Dr. Pedro Tavares Paes Lopes

Durante o desenvolvimento deste trabalho o autor recebeu auxílio financeiro da CAPES e CNPq

São Paulo, julho de 2019 



\section{A Lei de Weyl para o Laplaciano}

Esta versão da dissertação contém as correções e alterações sugeridas pela Comissão Julgadora durante a defesa da versão original do trabalho, realizada em 26/06/2019. Uma cópia da versão original está disponível no

Instituto de Matemática e Estatística da Universidade de São Paulo.

Comissão Julgadora:

- Prof. Dr. Pedro Tavares Paes Lopes (orientador) - IME-USP

- Prof. Dr. Gaetano Siciliano - IME-USP

- Prof. Dr. Marcus Antônio Mendonça Marrocos - UFABC 



\section{Agradecimentos}

Agradeço ao meu orientador Pedro pela sugestão do tema, pelas indicações bibliográficas, pelas recomendações de caminhos a seguir, pela revisão do texto e pela constante disposição em me atender.

Agradeço aos professores Gaetano e Marcus pela leitura realizada, pelos comentários feitos e pelas sugestões de alteração.

Agradeço aos meus familiares e amigos que me acompanharam durante esses anos e sempre me deram suporte e estímulo para eu seguir meus projetos.

Agradeço à minha companheira Aline que enfrenta comigo o dia a dia. 


\section{Resumo}

Neves, R. M. A Lei de Weyl para o Laplaciano. 2019. 93 p. Dissertação (Mestrado)

- Instituto de Matemática e Estatística, Universidade de São Paulo, São Paulo, 2019.

Demonstramos a Lei de Weyl sobre o comportamento assintótico dos autovalores do operador Laplaciano com condições de contorno de Dirichlet em domínios limitados e suaves com o auxílio do núcleo do calor. Para isso, fazemos um estudo dos operadores não-limitados, semigrupos e da transformada de Fourier. Por fim, expomos alguns resultados posteriores motivados pelo artigo de Mark Kac "Can one hear the shape of a drum?".

Palavras-chave: Análise espectral, núcleo do calor, semigrupos. 


\section{Abstract}

Neves, R. M. The Weyl Law for the Laplacian. 2019. 93 p. Dissertation (Master's degree) - Institute of Mathematics and Statistics, University of Sao Paulo, Sao Paulo, 2019 .

We prove the Weyl Law on the asymptotic behavior of eigenvalues of the Laplace operator with Dirichlet boundary conditions in smooth bounded domains with the help of the heat kernel. To that end, we study unbounded operators, semigroups and the Fourier transform. Lastly, we mention some further results motivated by Mark Kac's article "Can one hear the shape of a drum?".

Keywords: Spectral analysis, heat kernel, semigroups. 


\section{Sumário}

1 Introdução 1

2 Operadores não-contínuos $\quad 9$

2.1 Operadores fechados . . . . . . . . . . . . . . . . 11

2.2 O resolvente . . . . . . . . . . . . . . . . . . . . 15

2.3 Operadores com resolvente compacto . . . . . . . . . . . . . 18

2.4 Operadores não-contínuos em espaços de Hilbert . . . . . . . . . . . . . . . 22

2.5 Operadores de multiplicação . . . . . . . . . . . . . . . . . . . . 28

2.6 Teorema espectral . . . . . . . . . . . . . . . . . . . 32

3 Semigrupos $\quad 35$

3.1 Semigrupos fortemente contínuos . . . . . . . . . . . . 35

3.2 Semigrupos autoadjuntos . . . . . . . . . . . . . . . . . . . . . . . . 42

4 Transformada de Fourier $\quad 45$

4.1 Propriedades básicas . . . . . . . . . . . . . . . . . . . 45

4.2 Espaço de Schwartz . . . . . . . . . . . . . . . . . 50 50

4.3 Distribuições temperadas . . . . . . . . . . . . . . . . . 53

5 A Lei de Weyl $\quad 63$

5.1 O Laplaciano em $\mathbb{R}^{n}$ e em $\Omega$, com condições de contorno de Dirichlet . . . 63

5.2 Operadores de Hilbert-Schmidt . . . . . . . . . . . . . . . . . . 68

5.3 Monotonicidade . . . . . . . . . . . . . . . . . . 72

5.4 Teorema de Karamata . . . . . . . . . . . . . . . . . . . 76

5.5 Teorema de Weyl . . . . . . . . . . . . . . . . . . . . 79

6 Considerações finais $\quad 87$ 


\section{Introdução}

"Podemos ouvir a forma de um tambor?". Esta provocante questão é o título do famoso artigo de Mark Kac publicado em 1966 [28]. No artigo a pergunta se mantém aberta, mas o problema proposto dá origem a uma discussão fecunda que responde à indagação original, a reformula e produz novos resultados e interrogações ainda não esclarecidas. Antes de revelar a resposta objetiva para a pergunta e descrever alguns resultados posteriores, vamos nos ater ao significado da questão e sua interpretação matemática. Uma interpretação direta para a questão é se é possível determinar o formato de uma membrana a partir do som que ela emite ao vibrar. Em outras palavras, se um som carrega consigo informações suficientes para determinar o formato da membrana que o originou.

Para entender melhor o significado matemático, vamos estudar o problema da membrana circular vibrante. Matematicamente, uma membrana nada mais é, em duas dimensões, do que uma região do plano limitada por uma fronteira. Seja então $\Omega$ um disco de raio um e centro na origem e $\partial \Omega$ sua fronteira. Vamos imaginar que $\Omega$ define uma membrana elástica idealizada sem espessura que está fixada na sua fronteira $\partial \Omega$. O valor $u(t, x, y)$ representa o deslocamento transversal da membrana em relação ao seu plano de repouso no instante $t$ e no ponto com coordenadas cartesianas $x$ e $y$. Isto é, $u$ é a função que descreve o movimento perpendicular da membrana. A membrana está fixada em sua fronteira, isto é, $u(t, x, y)=0$ se $x^{2}+y^{2}=1$. Esta condição é conhecida como condição de contorno de Dirichlet.

A vibração da membrana respeita as leis da Física e seu comportamento é governado pela equação da onda

$$
u_{t t}=c^{2}\left(u_{x x}+u_{y y}\right)
$$

O símbolo $u_{t}$ significa a derivada parcial de $u$ com relação a variável $t$, que nada mais é do que uma medida que indica o quanto $u$ varia em determinado ponto e instante, se variarmos $t$. Corresponde a velocidade de um ponto da membrana em determinado instante. O lado esquerdo da equação $u_{t t}$ representa a aceleração de um ponto da membrana em um determinado instante e a parte $\left(u_{x x}+u_{y y}\right)$ do lado direito da equação representa a curvatura da membrana em determinado ponto e instante. A equação nos diz que a força de restauração e a curvatura em determinado ponto e instante são proporcionais, isto é, quanto mais curva a membrana estiver, maior será a força pra desfazer essa curvatura. 
Por fim, $c$ é a velocidade com que a vibração se propaga na membrana. O valor de $c$ é constante e depende das características físicas da membrana como espessura, densidade e tensão. Como $c$ é constante, podemos mudar as unidades de medida fazendo $c=1$. Assim, ficamos com a equação

$$
u_{t t}=u_{x x}+u_{y y}
$$

A parte $\left(u_{x x}+u_{y y}\right)$ da equação também é conhecida como o Laplaciano de $u$ e é representado pelo símbolo $\Delta u$. Desta forma, obtemos o seguinte problema

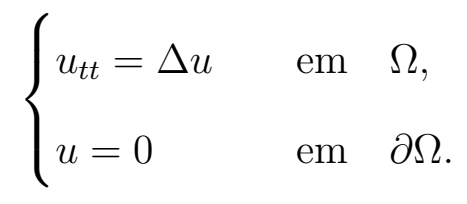

Resolver esse problema significa encontrar as funções $u$ que satisfazem essas condições. A equação da onda possui a solução trivial $u=0$ que representa uma membrana em estado de repouso. Se forem informadas a posição inicial da membrana $u(0, x, y)$ e a velocidade inicial $u_{t}(0, x, y)$, então o problema terá uma única solução.

Devido ao formato circular de nossa membrana, é conveniente utilizar coordenadas polares $r$ e $\theta$ para as variáveis espaciais de $u$. Em vez de localizarmos um ponto do plano pelas suas projeções em dois eixos perpendiculares, descrevemos sua posição com duas outras informações: a distância $r$ do ponto à origem e o ângulo $\theta$ em relação a um dos eixos. Os dois sistemas de coordenadas se associam pela relação

$$
x=r \sin \theta, \quad y=r \cos \theta .
$$

Assim a função $u$ passa a depender de $(t, r, \theta)$. Utilizando a relação que associa os dois sistemas de coordenadas e a regra da cadeia, podemos verificar que em coordenadas polares a equação da onda fica

$$
u_{t t}=u_{r r}+\frac{u_{r}}{r}+\frac{u_{\theta \theta}}{r^{2}} .
$$

Para resolver essa equação utilizamos o método de separação das variáveis, que procura por soluções da forma

$$
u(t, r, \theta)=T(t) R(r) \Theta(\theta) .
$$


As soluções dessa forma representam os modos normais de vibração da membrana, que são os padrões de movimento em que todas as partes da membrana se movem sinusoidalmente com a mesma frequência. Essas frequências características dos modos normais correspondem aos tons puros que a membrana é capaz de emitir. Substituindo a Função (3) na Equação (2) e dividindo por $T(t) R(r) \Theta(\theta)$, obtemos

$$
\frac{T^{\prime \prime}(t)}{T(t)}=\frac{R^{\prime \prime}(r)}{R(r)}+\frac{R^{\prime}(r)}{r R(r)}+\frac{\Theta^{\prime \prime}(\theta)}{r^{2} \Theta(\theta)} .
$$

A equação acima vale para todos os valores de $t, r$ e $\theta$. Como o lado esquerdo da equação depende apenas da variável $t$ e o lado direito não depende de $t$, logo, para todo $t$, o valor $\frac{T^{\prime \prime}(t)}{T(t)}$ é constante. Manipulando os termos da equação e utilizando o mesmo raciocínio, obtemos três equações diferenciais ordinárias

$$
\begin{aligned}
T^{\prime \prime}(t)+\lambda^{2} T(t) & =0, \\
\Theta^{\prime \prime}(\theta)+m^{2} \Theta(\theta) & =0, \\
r^{2} R^{\prime \prime}(r)+r R^{\prime}(r)+\left(\lambda^{2} r^{2}-m^{2}\right) R(r) & =0,
\end{aligned}
$$

onde $\lambda$ e $m$ são números reais positivos.

As soluções das duas primeiras equações são

$$
\begin{aligned}
T(t) & =A \cos (\lambda t)+B \sin (\lambda t), \\
\Theta(\theta) & =C \cos (m \theta)+D \sin (m \theta),
\end{aligned}
$$

onde $A, B, C$, e $D$ são constantes. O fato da função $\Theta$ ser periódica com período $2 \pi$ implica que $m$ é um número natural.

Fazendo a mudança de escala $\rho=\lambda r$ e considerando $R$ como uma função de $\rho$, podemos reescrever a terceira equação da forma

$$
\rho^{2} R^{\prime \prime}(\rho)+\rho R^{\prime}(\rho)+\left(\rho^{2}-m^{2}\right) R(\rho)=0 .
$$

Esta é a conhecida equação diferencial de Bessel. Essa equação possui duas famílias de soluções $J_{m}$ e $Y_{m}$, chamadas funções de Bessel. A família de funções $Y_{m}$ possui uma singularidade na origem e como sabemos que no nosso problema a solução deve estar 


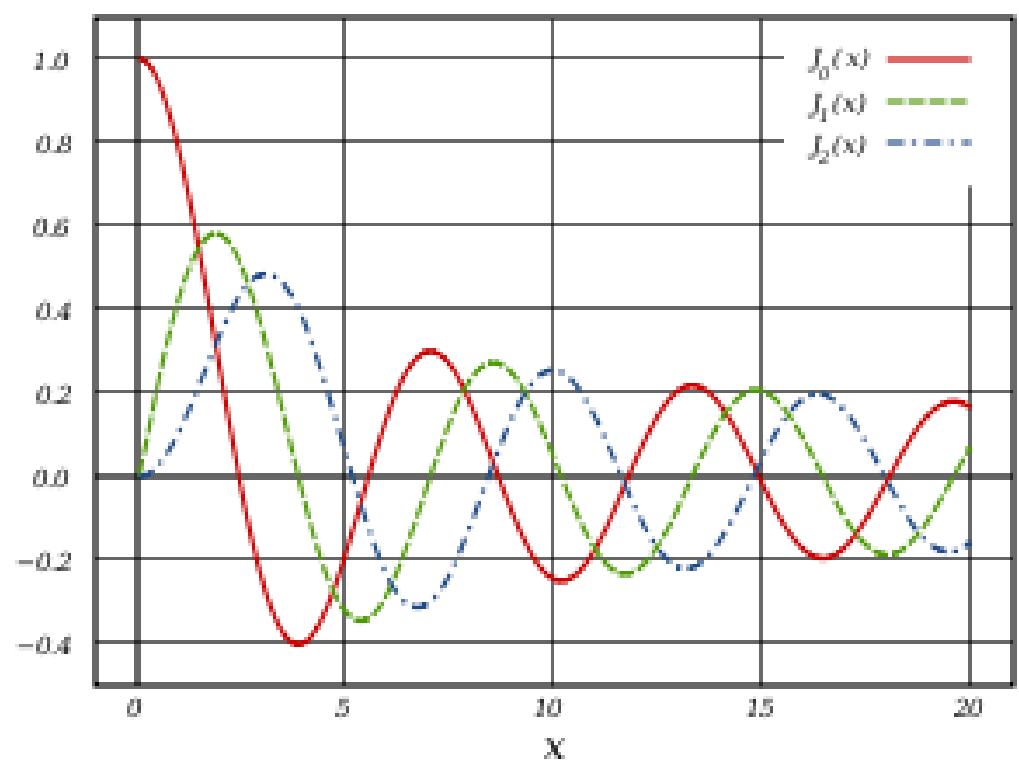

Figura 1: Funções de Bessel $J_{m}$ para $m=0,1,2$ [49].

definida na origem, então ficamos apenas com a família $J_{m}$ que é definida pela série

$$
J_{m}(\rho)=\sum_{n=0}^{\infty} \frac{(-1)^{n}}{n ! \Gamma(n+m+1)}\left(\frac{\rho}{2}\right)^{2 n+m} .
$$

onde $\Gamma$ é a função Gama, uma extensão da função fatorial para valores reais e complexos. Como a medida do raio da nossa membrana é um, a função $R$ deve satisfazer $R(1)=0$, $\operatorname{logo} \lambda$ precisa ser raiz da função de Bessel. Finalmente, juntando os resultados obtidos, temos a solução

$$
u(t, r, \theta)=\left(A \cos \lambda_{m n} t+B \sin \lambda_{m n} t\right) J_{n}\left(\lambda_{m n} r\right)(C \cos n \theta+D \sin n \theta),
$$

onde $\lambda_{m n}$ é a enésima raiz da função de Bessel $J_{m}$ e $m$ e $n$ são números naturais. Portanto, existem infinitas soluções.

Cada uma delas representa um modo normal de vibração da membrana, que tem uma frequência angular característica $\lambda_{m n}$. O modo fundamental de vibração de uma membrana é o modo normal de menor frequência, onde todas as partes da membrana se deslocam juntas para cima e para baixo. A nota que escutamos corresponde a frequência do modo fundamental. Nos demais modos normais, partes da membrana sobem e descem alternadamente, formando linhas nodais onde a membrana permanece em repouso. As frequências desses modos correspondem a série harmônica do tom fundamental. 

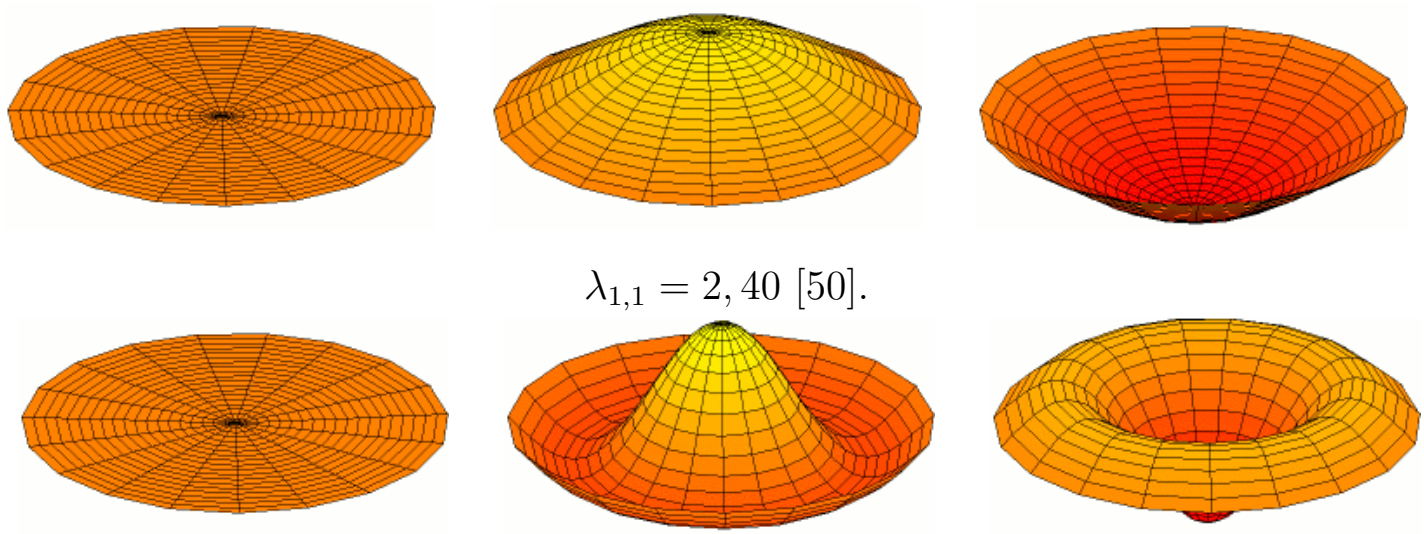

$$
\lambda_{1,2}=5,52[51]
$$
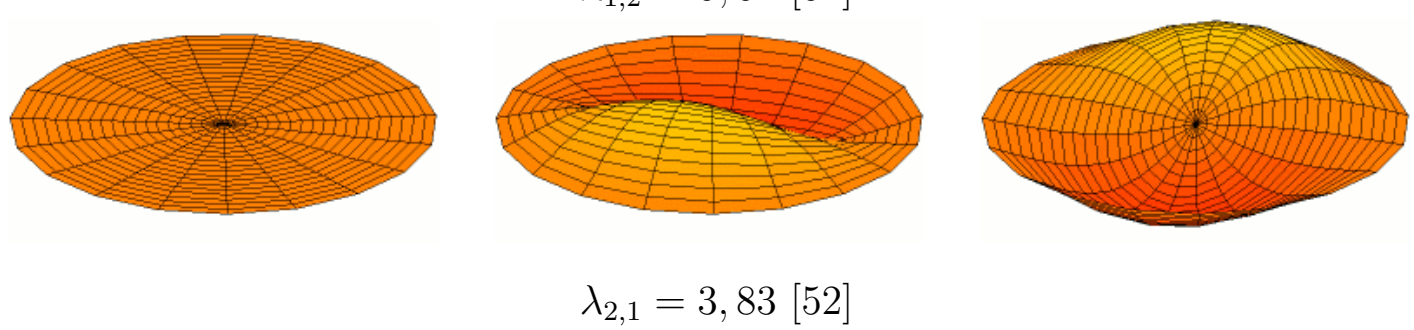

Figura 2: Cada linha corresponde a três instantes do modo normal de vibração correspondente a frequência angular $\lambda_{m n}$.

O conjunto das frequências de todos os modos normais de uma membrana, a menos de uma constante, se chama espectro. No caso da membrana circular de raio um, seu espectro é formado por todas as raízes das funções de Bessel $J_{m}$ onde $m$ é um número natural.

Agora estamos em condições de formular o sentido matemático da pergunta inicial. O que Kac quer saber é se conhecendo o espectro de um domínio $\Omega$ podemos determinar seu formato.

Em 1992, Carolyn Gordon e David Webb [22] responderam negativamente a pergunta feita por Kac. Eles apresentaram um exemplo de duas regiões do plano com formatos diferentes, mas com o mesmo espectro, chamadas isoespectrais. Isto significa que tabores com estes formatos, feitos do mesmo material e com mesma espessura e tensão, produzem o mesmo som.

Apesar de Gordon e Webb darem uma resposta objetiva a questão levantada por Kac, o tema continuou a ser investigado. A pergunta inicial poderia ser reformulada pela seguinte: quais características da geometria de um tambor podemos escutar e sob quais condições? Um fato extraordinário já conhecido, descoberto em 1911 por Hermann Weyl [48], conhecido como a Lei de Weyl, afirma que podemos escutar a área de um 


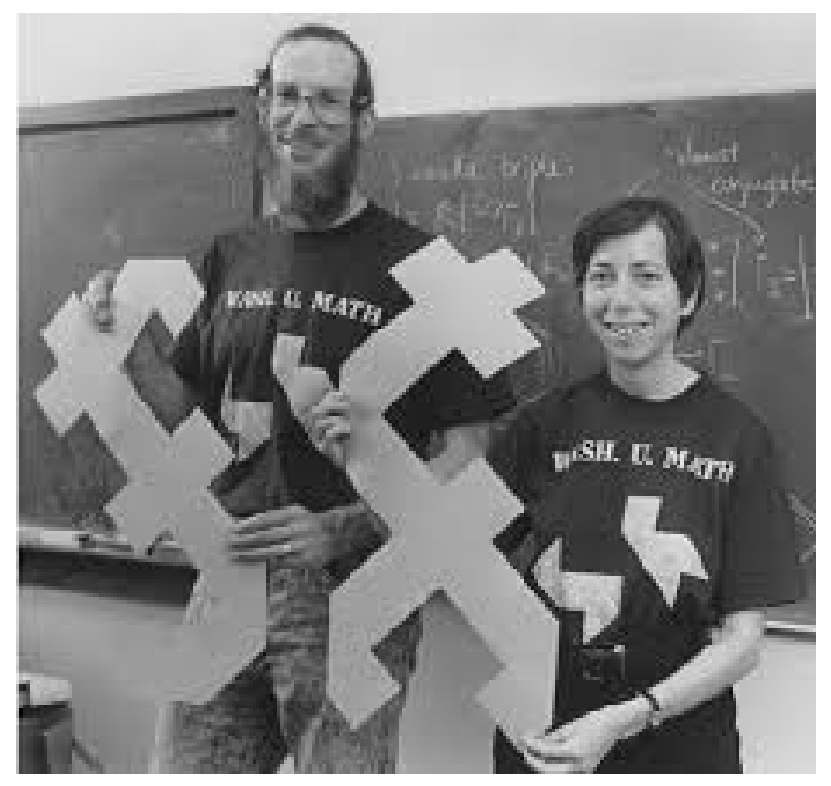

Figura 3: Webb e Gordon com as regiões isoespectrais descobertas [47].

tambor. Mais especificamente, ela descreve o comportamento assintótico dos autovalores do Laplaciano de Dirichlet. Os autovalores do Laplaciano de Dirichlet são os valores $\lambda$ para os quais existe uma função $u$ diferente de zero tal que

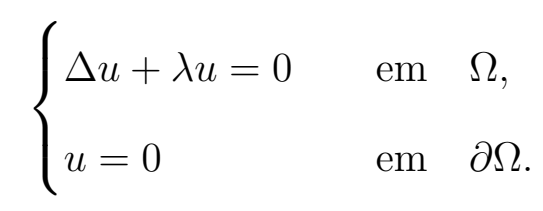

Neste problema a função $u$ só possui variáveis espaciais. No caso bidimensional, reescrevendo a equação acima, temos

$$
\frac{\Delta u(x, y)}{u(x, y)}=-\lambda .
$$

No problema da Equação da Onda (1), utilizando o método da separação das variáveis, fazendo $u(t, x, y)=X(x, y) T(t)$, temos

$$
\frac{T^{\prime \prime}(t)}{T(t)}=\frac{\Delta X(x, y)}{X(x, y)}=-f^{2}
$$

onde $f$ é a frequência angular dos modos normais do problema da Equação da Onda. Comparando as duas últimas equações, podemos notar que para uma mesma região $\Omega$, os autovalores do Laplaciano de Dirichlet $\lambda$ são iguais ao quadrado das frequências angulares $f$ dos modos normais do problema da Equação da Onda. Isto é, conhecendo um sabemos o outro. Para regiões $\Omega$ limitadas os autovalores são enumeráveis e podemos ordená-los 
da forma $0<\lambda_{1} \leq \lambda_{2} \leq \lambda_{3} \leq \ldots$. A lei de Weyl afirma que

$$
\lim _{\lambda \rightarrow \infty} \frac{N(\lambda)}{\lambda^{d / 2}}=\frac{\omega_{d}}{(2 \pi)^{d}}|\Omega|
$$

onde $d$ é a dimensão, $\omega_{d}$ é o volume da bola unitária de dimensão $d, N(\lambda)$ é a quantidade de autovalores menores ou iguais a $\lambda_{n}$ e $|\Omega|$ é a área de $\Omega$. Resumindo, a Lei de Weyl nos diz que se conhecermos os autovalores do Laplaciano, podemos determinar a área da região $\Omega$. Mas conhecer os autovalores do Laplaciano é a mesma coisa que conhecer as frequências dos modos normais de vibração da membrana delimitada pela região $\Omega$. Como o som de uma membrana está relacionado com a frequência de vibração, então a frase que diz que é possível escutar a área de um tambor ganha sentido.

A Lei de Weyl é o objeto central deste trabalho. Buscamos fazer uma demonstração detalhada da Lei de Weyl para o operador Laplaciano com condições de contorno de Dirichlet em domínios limitados e suaves. Seguimos a estrutura da demonstração encontrada em [2] e [13], que utiliza o núcleo do calor.

O trabalho está organizado em 6 capítulos contando com esta introdução. Os capítulos 2, 3 e 4 reúnem resultados básicos da teoria dos operadores não-limitados, semigrupos e da transformada de Fourier. A Lei de Weyl é demonstrada no capítulo 5 e, por fim, no capítulo 6, apresentamos alguns resultados posteriores motivados pelo artigo de Mark Kac "Can one hear the shape of drum?".

O presente trabalho foi realizado com apoio do Conselho Nacional de Desenvolvimento Científico e Tecnológico (CNPq) e da Coordenação de Aperfeiçoamento de Pessoal de Nível Superior - Brasil (CAPES) - Código de Financiamento 001. 


\section{Operadores não-contínuos}

Neste capítulo fazemos um breve estudo dos operadores não-contínuos, iniciando com o estudo dos operadores fechados. Na segunda e terceira seções, investigamos as propriedades espectrais dando ênfase aos operadores com resolvente compacto. Em seguida, analisamos os operadores autoadjuntos. Os operadores de multiplicação são utilizados como exemplo e suas propriedades são examinadas na seção cinco. Por fim, demonstramos o Teorema Espectral para operadores autoadjuntos, dissipativos e com resolvente compacto, nosso principal resultado, que utilizaremos posteriormente para representar o Laplaciano como um operador diagonal. A principal referência para este capítulo é [2].

A seguir apresentamos notações, definições e resultados preliminares com o intuito de servir de referência, já que serão utilizados posteriormente no texto. Uma exposição minuciosa pode ser encontrada em [7].

Denotamos por $\mathbb{N}$ o conjunto dos números naturais sem o zero, $\mathbb{R}$ o conjunto dos números reais e $\mathbb{C}$ o conjunto dos números complexos. O espaço Euclidiano usual de dimensão $n$ é denotado por $\mathbb{R}^{n}=\left\{\left(x_{1}, x_{2}, \ldots, x_{n}\right): x_{1}, x_{2}, \ldots, x_{n} \in \mathbb{R}\right\}$. Caso não explicitado, $\Omega$ é um aberto de $\mathbb{R}^{n}$. Denotamos $C(\Omega)=\{f: \Omega \rightarrow \mathbb{C}: f$ é contínua $\}, C^{k}(\Omega)$ o espaço das funções com derivadas parciais de ordem $k$ contínuas e $C^{\infty}(\Omega)=\cap_{k \in \mathbb{N}} C^{k}(\Omega)$ o espaço das funções suaves. Ao longo do texto, caso não explicitado, $X$ denota um espaço de Banach complexo e $H$ um espaço de Hilbert separável e complexo.

Definição 2.1. (Operador)

Sejam $X$ e $Y$ espaços de Banach. Um operador de $X$ em $Y$ é uma função linear $A: D(A) \subset X \rightarrow Y$, cujo domínio $D(A)$ é um subespaço linear de $X$. Se $A$ é um operador de $X$ em $X$, então dizemos que $A$ é um operador em $X$.

Definição 2.2. (Gráfico, núcleo e imagem de um operador)

Seja $A$ um operador de $X$ em $Y$. O gráfico, o núcleo e a imagem do operador $A$ são, respectivamente, os conjuntos definidos por

$$
\begin{aligned}
G(A) & :=\{(x, A x) \in D(A) \times Y: x \in D(A)\}, \\
\operatorname{ker}(A) & :=\{x \in D(A): A x=0\}, \\
\operatorname{im}(A) & :=\{A x \in Y: x \in D(A)\} .
\end{aligned}
$$

Definição 2.3. (Operador contínuo) Um operador $A$ de $X$ em $Y$ é contínuo em $x_{0} \in X$ 
se para todo $\epsilon>0$, existe $\delta>0$ tal que se $x \in D(A)$ e $\left\|x-x_{0}\right\|<\delta$, então $\left\|A x-A x_{0}\right\|<\epsilon$. Dizemos que o operador $A$ é contínuo se ele for contínuo para todo ponto de seu domínio.

Definição 2.4. I denota o operador identidade e $\mathcal{L}(X, Y)$ o conjunto dos operadores contínuos de $X$ em $Y$, tais que $D(A)=X$. Denotamos $\mathcal{L}(X, X)$ por $\mathcal{L}(X)$.

Proposição 2.5. (Caracterização dos operadores contínuos)

Seja A um operador de $X$ em $Y$, tal que $D(A)=X$. As seguintes afirmações são equivalentes:

(a) A é contínuo.

(b) A é contínuo na origem.

(c) A é contínuo em algum ponto em $X$.

(d) A é limitado, isto é, $\sup \{\|A x\|: x \in X$ e $\|x\| \leq 1\}<\infty$.

(e) Existe $M \in[0, \infty)$ tal que $\|A x\| \leq M\|x\|$, para todo $x \in X$.

Definição 2.6. (Extensão de um operador)

Sejam $A$ e $B$ operadores em $X$. $B$ é uma extensão de $A$ se $D(A) \subset D(B)$ e $A x=B x$, para todo $x \in D(A)$. Neste caso, escrevemos $A \subset B$. $A$ é igual a $B$ quando $A \subset B$ e $B \subset A$, ou seja, $D(A)=D(B)$ e $A x=B x$, para todo $x \in D(A)$.

Teorema 2.7. (Teorema da transformação linear limitada)

Seja A um operador limitado em $X$. Então A pode ser estendido de forma única para um operador limitado $\tilde{A}: \overline{D(A)} \rightarrow X$.

Teorema 2.8. (Teorema de Banach-Steinhaus)

Sejam $X$ e $Y$ espaços de Banach e $\mathcal{A}$ uma coleção de operadores em $\mathcal{L}(X, Y)$ tal que, para todo $x \in X$, temos

$$
\sup _{A \in \mathcal{A}}\|A x\|<\infty
$$

então

$$
\sup _{A \in \mathcal{A}}\|A\|<\infty .
$$

Teorema 2.9. (Teorema do Gráfico Fechado)

Se $X$ e $Y$ são espaços de Banach e $A: X \rightarrow Y$ é um operador linear, então $A$ é contínuo se, e somente se, $G(A)$ é fechado em $X \times Y$. O resultado também vale para 
espaços de Fréchet (a definição precisa e resultados sobre espaços de Fréchet pode ser consultada em [24]).

\subsection{Operadores fechados}

Muitos operadores importantes, como o Laplaciano, não são contínuos. Entretanto possuem certas propriedades mais fracas que permitem que se faça alguma análise. Nos referimos aos operadores fechados, que são estudados nesta seção.

Definição 2.10. (Operador fechado)

Um operador $A$ em $X$ é fechado se para toda sequência $\left(x_{n}\right)_{n \in \mathbb{N}}$ em $D(A)$ tal que $x_{n} \rightarrow x \in X$ e $A x_{n} \rightarrow y \in X$, temos $x \in D(A)$ e $A x=y$. Em outras palavras, se $\left(x_{n}\right)_{n \in \mathbb{N}}$ é uma sequência em $D(A)$ e os limites $\lim _{n \rightarrow \infty} x_{n}, \lim _{n \rightarrow \infty} A x_{n}$ existem, então $A\left(\lim _{n \rightarrow \infty} x_{n}\right)=$ $\lim _{n \rightarrow \infty} A x_{n}$.

Em seguida, obtemos outras caracterizações para operadores fechados. Para isso, definimos a norma do gráfico.

Definição 2.11. (Norma do gráfico)

A norma do gráfico $\|\cdot\|_{A}: D(A) \rightarrow[0, \infty)$ de um operador $A$ em $X$ é definida por

$$
\|x\|_{A}:=\|x\|+\|A x\|
$$

É fácil verificar que a norma do gráfico de fato define uma norma.

Proposição 2.12. (Caracterizações dos operadores fechados)

Seja $[D(A)]$ o subespaço $D(A)$ equipado com a norma do gráfico $\|x\|_{A}$. As seguintes afirmações são equivalentes:

(a) A é um operador fechado,

(b) O gráfico de $A$ é um subespaço fechado de $X \times X$,

(c) $[D(A)]$ é um espaço de Banach.

Demonstração.

$(a) \Longleftrightarrow(b)$ Pela definição, $A$ é fechado se, e somente se, para toda sequência $\left(x_{n}, A x_{n}\right)_{n \in \mathbb{N}}$ em $G(A)$, tal que $\left(x_{n}, A x_{n}\right) \rightarrow(x, y) \in X \times X$, temos $(x, y) \in G(A)$, que 
por sua vez é equivalente a dizer que $G(A)$ é fechado.

$(b) \Longleftrightarrow(c)$ Como $\|(x, A x)\|_{X \times X}=\|x\|+\|A x\|=\|x\|_{A}$, então $G(A)$ é fechado se, e somente se, $\left(G(A),\|\cdot\|_{X \times X}\right)$ é completo, já que $X \times X$ é um espaço completo. O que equivale a dizer que $[D(A)]$ é completo.

É de imediato que todo operador contínuo é fechado. Adiante, segue um exemplo de um operador não-contínuo e fechado e um não-fechado.

Exemplo 2.13. (Operador fechado e operador não-fechado)

Seja $A: D(A) \subset C([0,1]) \ni f \mapsto f^{\prime} \in C([0,1])$, onde $D(A)=C^{1}([0,1])$. O operador $A$ não é contínuo. De fato, seja $f_{n}=n^{-1 / 2} \sin (n x)$, para todo $n \in \mathbb{N}$. Temos $f_{n} \in C^{1}([0,1])$, $f_{n} \rightarrow 0$ e $\left\|A f_{n}\right\| \geq\left|f_{n}^{\prime}(0)\right|=\sqrt{n} \rightarrow \infty$. Porém $A$ é fechado, pois se $\left(f_{n}\right)_{n \in \mathbb{N}}$ é uma sequência de funções em $C^{1}([0,1])$ tal que $f_{n} \rightarrow f \in C([0,1])$ e $A f_{n}=f_{n}^{\prime} \rightarrow g \in C([0,1])$, então $f \in C^{1}([0,1])$ e $f^{\prime}=g$.

Nas condições anteriores, mas mudando o domínio de $A$ para $C^{\infty}([0,1])$, o fato das sequências $\left(f_{n}\right)_{n \in \mathbb{N}}$ e $\left(f_{n}^{\prime}\right)_{n \in \mathbb{N}}$ convergirem para $f$ e $f^{\prime}$ não implica que $f \in C^{\infty}([0,1])$ e, portanto, $A$ não é fechado.

Abaixo seguem algumas propriedades úteis de operadores fechados.

Proposição 2.14. (Propriedades de operadores fechados)

(a) Se $D(A)$ é fechado, então A é fechado se, e somente se, A é contínuo.

(b) Se A é injetora, então A é fechado se, e somente se, $A^{-1}: i m(A) \rightarrow D(A)$ é fechado.

\section{Demonstração.}

(a) Segue direto do Teorema do Gráfico Fechado 2.9.

(b) O operador $A$ é fechado se, e somente se,

$$
G(A)=\{(x, A x): x \in D(A)\}=\left\{\left(A^{-1} y, y\right): y \in \operatorname{im}(A)\right\}
$$

é fechado. Isso é o mesmo que dizer que $G\left(A^{-1}\right)$ é fechado, que, por sua vez, é equivalente a dizer que $A^{-1}$ é fechado. 
Um operador pode não ser fechado porque seu domínio é pequeno. Ou seja, alguns operadores não-fechados são restrições de operadores fechados, isto é, possuem uma extensão fechada.

Definição 2.15. (Operador fechável e fecho)

$A$ é fechável se existe uma extensão fechada de $A$. O fecho $\bar{A}$ de $A$, caso exista, é a menor extensão fechada de $A$. Em outras palavras, $\bar{A}$ é o fecho de $A$ se: $\bar{A}$ é fechado, $A \subset \bar{A}$ e $\bar{A} \subset B$, para toda extensão fechada $B$ de $A$.

É possível estender uma função de diferentes maneiras. O próximo resultado mostra que se um operador possui uma extensão fechada, então existe a menor extensão fechada.

Proposição 2.16. (Fecho de operador fechável)

Se A é fechável, então o fecho $\bar{A}$ de $A$ existe.

\section{Demonstração.}

Seja $\bar{A}: D(\bar{A}) \rightarrow X$ a função definida por $\bar{A}(x)=y$, onde

$$
D(\bar{A}):=\left\{x \in X: \text { existe }\left(x_{n}\right)_{n \in \mathbb{N}} \text { em } D(A) \text { tal que }\left(x_{n}\right) \rightarrow x \in X \text { e }\left(A x_{n}\right) \rightarrow y \in X\right\}
$$

Precisamos mostrar que $\bar{A}$ está bem definida, isto é, dadas sequências $\left(x_{n}\right)_{n \in \mathbb{N}}$ e $\left(w_{n}\right)_{n \in \mathbb{N}}$ em $D(A)$ tais que $x_{n} \rightarrow x, w_{n} \rightarrow x, A x_{n} \rightarrow y, A w_{n} \rightarrow z$, temos $y=z$. De fato, como $A$ é fechável, existe uma extensão fechada $B$ de $A$, $\operatorname{logo} x \in D(B)$ e $B x=y=z$.

Segue do fato que $D(A)$ é um subespaço de $X$ e das propriedades de limites que $D(\bar{A})$ é subespaço de $X$ e $\bar{A}$ é linear. O operador $\bar{A}$ é uma extensão de $A$, com efeito, basta tomar $x \in D(A)$ e $x_{n}=x$, para todo $n \in \mathbb{N}$, na definição de $D(\bar{A})$.

Para mostrar que $\bar{A}$ é fechado considere uma sequência $\left(u_{n}, \bar{A} u_{n}\right)_{n \in \mathbb{N}}$ em $G(\bar{A})$ tal que $\left(u_{n}, \bar{A} u_{n}\right) \rightarrow(x, y) \in X \times X$. Para cada $n \in \mathbb{N}$, existe $x_{n} \in D(A)$ tal que

$$
\left\|u_{n}-x_{n}\right\|+\left\|\bar{A} u_{n}-A x_{n}\right\|<\frac{1}{n}
$$

Fazendo $n \rightarrow \infty$ verificamos que $x_{n} \rightarrow x$ e $A x_{n} \rightarrow y$. Logo, pela definição de $D(\bar{A})$, $(x, y) \in G(\bar{A})$.

Falta mostrar que $\bar{A}$ é a menor extensão fechável de $A$, deveras, se $x \in D(\bar{A})$, então existe uma sequência $\left(x_{n}\right)_{n \in \mathbb{N}}$ em $D(A) \operatorname{com} x_{n} \rightarrow x \in X$ e $A\left(x_{n}\right) \rightarrow y \in X$ e, para toda extensão fechável $B$ de $A$, temos $x \in D(B)$ e $B x=y=\bar{A} x$. 
A seguir, mostramos outras maneiras de identificar operadores fecháveis.

Proposição 2.17. (Caracterizações de operadores fecháveis)

As seguintes afirmações são equivalentes:

(a) A é fechável.

(b) Para toda sequência $\left(x_{n}\right)_{n \in \mathbb{N}}$ em $D(A)$, tal que $\left(x_{n}, A x_{n}\right) \rightarrow(0, y)$, temos $y=0$.

(c) Existe um operador fechado $B$ em $X$ tal que $\overline{G(A)}=G(B)$. Neste caso $\bar{A}=B$.

Demonstração.

$(a) \Longrightarrow(b)$ Seja $\left(x_{n}\right)_{n \in \mathbb{N}}$ em $D(A)$, tal que $\left(x_{n}, A x_{n}\right) \rightarrow(0, y)$. A é fechável, portanto $\bar{A} 0=y=0$.

$(b) \Longrightarrow(c)$ Seja $B: D(B) \rightarrow X$ a função definida por $B(x)=y$, onde

$$
D(B):=\{x \in X: \text { existe } y \in X \text { tal que }(x, y) \in \overline{G(A)}\}
$$

$\log O G(B)=\overline{G(A)}$.

Primeiro verificamos que $D(B)$ é um subespaço de $X$. Para isso, considere $x, w \in$ $D(B)$ e $\lambda \in \mathbb{C}$. Pela definição de $D(B)$, existem $y, z \in X$ e sequências $\left(x_{n}, A x_{n}\right)_{n \in \mathbb{N}}$, $\left(w_{n}, A w_{n}\right)_{n \in \mathbb{N}}$ em $G(A)$ tais que $\left(x_{n}, A x_{n}\right) \rightarrow(x, y) \in \overline{G(A)}$ e $\left(w_{n}, A w_{n}\right) \rightarrow(w, z) \in \overline{G(A)}$. Como $D(A)$ é um espaço linear, então $\left(\lambda x_{n}+u_{n}, A\left(\lambda x_{n}+u_{n}\right)\right) \in G(A)$, para todo $n \in \mathbb{N}$, $\mathrm{e}\left(\lambda x_{n}+u_{n}, A\left(\lambda x_{n}+u_{n}\right)\right) \rightarrow(\lambda x+u, \lambda y+z) \in \overline{G(A)}$. Logo, $(\lambda x+y) \in D(B)$.

Precisamos mostrar que $B$ está bem definida, isto é, se $\left(x, y_{1}\right),\left(x, y_{2}\right) \in \overline{G(A)}$, então $y_{1}=y_{2}$. De fato, $\left(x, y_{1}\right)-\left(x, y_{2}\right)=\left(0, y_{1}-y_{2}\right) \in \overline{G(A)}$, então existe uma sequência $\left(x_{n}\right)_{n \in \mathbb{N}}$ em $D(A) \operatorname{com} x_{n} \rightarrow 0$ e $A x_{n} \rightarrow y_{1}-y_{2}$. Pela hipótese, temos $y_{1}-y_{2}=0, \operatorname{logo}$ $y_{1}=y_{2}$.

A linearidade de $B$ segue do fato que $D(B)$ é um subespaço de $X$ e das propriedades de limites.

É de imediato que $B$ é uma extensão de $A$.

Por fim, $B$ é fechado, já que seu gráfico $G(B)=\overline{G(A)}$ é fechado.

$(c) \Longrightarrow(a)$ Seja $B$ um operador fechado em $X$ tal que $\overline{G(A)}=G(B)$. Para todo $x \in D(A),(x, A x) \in G(A) \subset \overline{G(A)}=G(B)$. Logo $x \in D(B)$ e $A x=B x$, para todo 
$x \in D(A)$. Portanto $B$ é uma extensão fechada de $A$ e $A$ é fechável.

Para mostrar que $B$ é o fecho de $A$, suponha $C$ uma extensão fechada de $A$ e considere $x \in D(B)$. Como $(x, B x) \in G(B) \subset \overline{G(A)}$, então existe uma sequência $\left(x_{n}, A x_{n}\right)_{n \in \mathbb{N}}$ em $G(A)$, tal que $\left(x_{n}, A x_{n}\right) \rightarrow(x, B x)$. Temos $G(A) \subset G(C)$, pois $C$ é extensão de $A$, portanto, como $C$ é fechado, $x \in D(C)$ e $B x=C x$.

Por fim, mostramos que nem todo operador não-fechado é fechável, como é o caso do exemplo abaixo.

Exemplo 2.18. (Operador não-fechável)

Seja $A$ o operador em $C([0,1])$, com $D(A)=C^{1}([0,1])$, que leva $f \in C^{1}([0,1])$ na função constante com valor igual a $f^{\prime}(0)$. Seja $f_{n}=n^{-1} \sin (n x)$, para todo $n \in \mathbb{N}$. Temos $f_{n} \in C^{1}([0,1]), f_{n} \rightarrow 0$ e $A f_{n}$ igual a função constante com valor igual a 1 , para todo $n \in \mathbb{N}$. Logo, $A f_{n} \rightarrow 1 \neq 0$ e, pela Proposição $2.17, A$ não é fechável.

\subsection{O resolvente}

Um operador não-contínuo $A$ pode ter inversa contínua. Assim, podemos utilizar resultados de operadores contínuos no estudo de $A$.

Definição 2.19. (Resolvente e espectro)

Seja $A$ um operador em $X$. O conjunto resolvente de $A$ é definido por

$$
\rho(A):=\left\{\lambda \in \mathbb{C}:(\lambda I-A): D(A) \rightarrow X \text { é bijetora e }(\lambda I-A)^{-1} \in \mathcal{L}(X)\right\} .
$$

A linearidade de $(\lambda I-A)^{-1}$ é automática se $\lambda I-A: D(A) \rightarrow X$ é bijetora. Para cada $\lambda \in \rho(A)$,

$$
R(\lambda, A):=(\lambda I-A)^{-1} \in \mathcal{L}(X)
$$

é chamado o resolvente de $A$ em $\lambda$. Escrevemos $(\lambda-A)$ como uma notação para $(\lambda I-A)$. O espectro de $A$ é o conjunto

$$
\sigma(A):=\mathbb{C} \backslash \rho(A)
$$

e o espectro pontual de $A$ é o conjunto dos autovalores de $A$,

$$
\sigma_{p}(A):=\{\lambda \in \mathbb{C}: \exists x \in D(A), x \neq 0, A x=\lambda x\}
$$


Abaixo seguem algumas propriedades úteis que relacionam operadores fechados com resolventes.

Proposição 2.20. (Operadores fechados e resolvente)

(a) Se $\lambda \in \mathbb{C}$, então A é fechado se, e somente se, $(\lambda-A)$ é fechado.

(b) Se $\rho(A) \neq \emptyset$, então A é fechado.

(c) Se $A$ é fechado e $(\lambda-A): D(A) \rightarrow X$ é bijetora, então $\lambda \in \rho(A)$.

Demonstração.

(a) $(\Longrightarrow)$ Seja $\left(x_{n}\right)_{n \in \mathbb{N}}$ uma sequência em $D(A)$ tal que $x_{n} \rightarrow x \in X$ e $(\lambda-A) x_{n} \rightarrow$ $y \in X$. Manipulando a última expressão temos $A x_{n} \rightarrow \lambda x-y$. Como $A$ é fechado, então $x \in D(\lambda-A)$ e $A x=\lambda x-y$ e, portanto, $(\lambda-A) x=y$.

$(\Longleftarrow)$ Seja $B=(\lambda-A)$. Se $B$ é fechado, então $(\lambda-B)=A$ é fechado.

(b) Pela hipótese, existe $\lambda \in \mathbb{C}$ tal que $(\lambda-A)$ é bijetora e $(\lambda-A)^{-1}$ é contínuo. Segue da Proposição 2.14 que $(\lambda-A)^{-1}$ é fechado e, consequentemente, $(\lambda-A)$ é fechado.

(c) Basta mostrar que $(\lambda-A)^{-1}$ é contínua. Pelas equivalências demonstradas anteriormente temos que $A$ é fechado $\stackrel{2.20(a)}{\Longleftrightarrow}(\lambda-A)$ é fechado $\stackrel{2.12(b)}{\Longleftrightarrow} G(\lambda-A)$ é fechado $\stackrel{2.14(b)}{\Longleftrightarrow} G\left((\lambda-A)^{-1}\right)$ é fechado $\stackrel{2.12(b)}{\Longleftrightarrow}(\lambda-A)^{-1}$ é fechado $\stackrel{2.14(a)}{\Longleftrightarrow}(\lambda-A)^{-1}$ é contínuo.

O próximo resultado nos revela, em particular, que o conjunto resolvente é sempre aberto.

\section{Proposição 2.21.}

Seja $A$ um operador em $X, \lambda_{0} \in \rho(A)$ e $\lambda \in \mathbb{C}$. Se $\left|\lambda-\lambda_{0}\right|<\left\|R\left(\lambda_{0}, A\right)\right\|^{-1}$, então $\lambda \in \rho(A) e$

$$
R(\lambda, A)=\sum_{n=0}^{\infty}\left(\lambda_{0}-\lambda\right)^{n} R\left(\lambda_{0}, A\right)^{n+1} \in \mathcal{L}(X) .
$$

Em particular, a função $\lambda \mapsto R(\lambda, A)$ é analítica.

Demonstração.

Temos

$$
\begin{aligned}
(\lambda-A) & =\left(\lambda-\lambda_{0}\right) R\left(\lambda_{0}, A\right)\left(\lambda_{0}-A\right)+\left(\lambda_{0}-A\right) \\
& =\left(I-\left(\lambda_{0}-\lambda\right) R\left(\lambda_{0}, A\right)\right)\left(\lambda_{0}-A\right) .
\end{aligned}
$$


Como $\left\|\left(\lambda_{0}-\lambda\right) R\left(\lambda_{0}, A\right)\right\|<1$, então a inversa de $\left(I-\left(\lambda_{0}-\lambda\right) R\left(\lambda_{0}, A\right)\right)$ existe e é dada pela série de Neumann

$$
\sum_{n=0}^{\infty}\left(\lambda_{0}-\lambda\right)^{n} R\left(\lambda_{0}, A\right)^{n} .
$$

$\operatorname{Logo} R(\lambda, A)=R\left(\lambda_{0}, A\right)\left(I-\left(\lambda_{0}-\lambda\right) R\left(\lambda_{0}, A\right)\right)^{-1}$ e $\lambda \in \rho(A)$.

\section{Corolário 2.22.}

$O$ conjunto resolvente é aberto e se $\left(\lambda_{n}\right)_{n \in \mathbb{N}}$ é uma sequência em $\rho(A), \lambda_{n} \rightarrow \lambda \in \mathbb{C}$ $e \sup _{n \in \mathbb{N}}\left\|R\left(\lambda_{n}, A\right)\right\|<\infty$, então $\lambda \in \rho(A)$.

\section{Demonstração.}

Pela hipótese existe $C>0$ tal que $\left\|R\left(\lambda_{n}, A\right)\right\|<C$, para todo $n \in \mathbb{N}$. Logo $1 /\left\|R\left(\lambda_{n}, A\right)\right\|>1 / C$, para todo $n \in \mathbb{N}$. Podemos tomar $\lambda_{n}$ tal que $\left|\lambda_{n}-\lambda\right|<1 / C$, $\log 0 \lambda \in \rho(A)$.

A seguir, vemos que existe uma relação direta entre o espectro de um operador e o espectro de seu resolvente.

Proposição 2.23. (Espectro de um operador e de seu resolvente)

Seja $\lambda_{0} \in \rho(A)$, então:

(a) $\lambda \in \sigma(A) \Longleftrightarrow\left(\lambda_{0}-\lambda\right)^{-1} \in \sigma\left(R\left(\lambda_{0}, A\right)\right)$. Em particular, se $\sigma(A)=\emptyset$, então $\sigma\left(R\left(\lambda_{0}, A\right)\right)=\{0\}$.

(b) $\lambda \in \sigma_{p}(A) \Longleftrightarrow\left(\lambda_{0}-\lambda\right)^{-1} \in \sigma_{p}\left(R\left(\lambda_{0}, A\right)\right)$

\section{Demonstração.}

(a) Seja $\mu \in \rho(A), \mu \neq \lambda_{0}$. Observe que

$$
I=\left[1-\left(\lambda_{0}-\mu\right) R\left(\lambda_{0}, A\right)\right]\left(\lambda_{0}-A\right) R(\mu, A) .
$$

Logo

$$
\left[\frac{1}{\lambda_{0}-\mu}-R\left(\lambda_{0}, A\right)\right]^{-1}=\left(\lambda_{0}-\mu\right)\left(\lambda_{0}-A\right) R(\mu, A)
$$

e

$$
(\mu-A)=\left(\lambda_{0}-\mu\right)\left[\left(\lambda_{0}-\mu\right)^{-1}-R\left(\lambda_{0}, A\right)\right]\left(\lambda_{0}-A\right) .
$$


$(\Longrightarrow)$ Seja $\lambda \in \sigma(A)$ e suponha $\left(\lambda_{0}-\lambda\right)^{-1} \notin \sigma\left(R\left(\lambda_{0}, A\right)\right) . \quad \operatorname{Logo}\left(\lambda_{0}-\lambda\right)^{-1} \in$ $\rho\left(R\left(\lambda_{0}, A\right)\right)$ a Equação $(7)$ implica que $\lambda \in \rho(A)$, pois

$$
R(\lambda, A)=\left(\lambda_{0}-\mu\right)^{-1} R\left(\lambda_{0}, A\right) R\left[\left(\lambda_{0}-\mu\right)^{-1}, R\left(\lambda_{0}, A\right)\right] .
$$

$(\Longleftarrow)$ Seja $\left(\lambda_{0}-\lambda\right)^{-1} \in \sigma\left(R\left(\lambda_{0}, A\right)\right)$ e suponha $\lambda \notin \sigma(A)$. Logo $\lambda \in \rho(A)$ e a Equação (6) com $\mu=\lambda$ implica que $\left(\lambda_{0}-\lambda\right)^{-1} \in \rho\left(R\left(\lambda_{0}, A\right)\right)$.

(b) $\lambda \in \sigma_{p}(A) \Longleftrightarrow \exists x \neq 0: \lambda x=A x \Longleftrightarrow\left(\lambda_{0}-\lambda\right) x=\left(\lambda_{0}-A\right) x \Longleftrightarrow$ $\left(\lambda_{0}-\lambda\right)^{-1} x=R\left(\lambda_{0}, A\right) x \Longleftrightarrow\left(\lambda_{0}-\lambda\right)^{-1} \in \sigma_{p}\left(R\left(\lambda_{0}, A\right)\right)$.

Por último, obtemos a identidade do resolvente que, em particular, mostra que resolventes de um mesmo operador comutam.

Proposição 2.24. (Identidade do resolvente)

Para todo $\lambda, \mu \in \rho(A)$, vale:

$$
R(\lambda, A)-R(\mu, A)=R(\lambda, A) R(\mu, A)(\mu-\lambda)
$$

Demonstração.

Temos

$$
\begin{aligned}
R(\lambda, A)-R(\mu, A) & =R(\lambda, A)[I-(\lambda-A) R(\mu, A)] \\
& =R(\lambda, A)[(\mu-A)-(\lambda-A)] R(\mu, A) \\
& =R(\lambda, A) R(\mu, A)(\mu-\lambda) .
\end{aligned}
$$

Como queríamos demonstrar.

\subsection{Operadores com resolvente compacto}

Iniciamos esta seção relembrando a definição e alguns resultados sobre a teoria dos operadores compactos. Uma exposição minuciosa pode ser encontrada em [7].

Definição 2.25. (Operador compacto)

Sejam $X, Y$ espaços de Banach e $B=\{x \in X:\|x\| \leq 1\}$. Um operador $A$ de $X$ em $Y$ é compacto se $\overline{A(B)}$ é um conjunto compacto em $Y$. Denotamos por $\mathcal{K}(X, Y)$ o conjunto de todos os operadores compactos de $X$ em $Y$ e $\mathcal{K}(X, X)=\mathcal{K}(X)$. 


\section{Proposição 2.26.}

Sejam $X, Y$ espaços de Banach. $\mathcal{K}(X, Y)$ é um subespaço fechado em $\mathcal{L}(X, Y)$.

Proposição 2.27. (Propriedade de ideal dos operadores compactos)

Sejam $X, Y, Z, W$ espaços de Banach, $A \in \mathcal{L}(X, Y), B \in \mathcal{L}(Z, W)$ e $C \in \mathcal{K}(Y, Z)$. Então $B C A \in \mathcal{K}(X, W)$.

\section{Proposição 2.28.}

Todo operador com posto finito é compacto e um operador A em um espaço de Hilbert é compacto se, e somente se, A é o limite de uma sequência de operadores com posto finito.

\section{Proposição 2.29.}

Seja A um operador compacto, então:

(a) $\sigma(A) \backslash\{0\}=\sigma_{p}(A) \backslash\{0\}$.

(b) $\sigma(A)$ é finito ou $\sigma(A)=\left\{\lambda_{n} \in \mathbb{C}: n \in \mathbb{N}\right\} \cup\{0\}$ e $\lambda_{n} \rightarrow 0$.

(c) $\operatorname{dim} \operatorname{ker}(\lambda-A)<\infty$, para todo $\lambda \in \mathbb{C} \backslash\{0\}$.

Essas são propriedades bem conhecidas dos operadores compactos. Nesta seção, verificamos as implicações dessas propriedades para os operadores com resolvente compacto, como o Laplaciano em domínios limitados, que estudaremos mais a frente.

\section{Definição 2.30.}

Um operador $A$ em $X$ tem resolvente compacto se $\rho(A) \neq \emptyset$ e $R(\lambda, A)$ é compacto, para todo $\lambda \in \rho(A)$.

Note que a Proposição 2.20 implica que todo operador com resolvente compacto é fechado. O próximo resultado mostra que se o resolvente de um operador é compacto em um ponto então ele é compacto em todos os pontos do conjunto resolvente.

Proposição 2.31. (Caracterizações de operadores com resolvente compacto)

Seja $\rho(A) \neq \emptyset$. As seguintes afirmações são equivalentes:

(a) A tem resolvente compacto.

(b) Existe $\lambda \in \rho(A)$ tal que $R(\lambda, A)$ é compacto. 
(c) A injeção canônica $i:[D(A)] \ni x \mapsto x \in X$ é compacta.

Demonstração.

$(a) \Longleftrightarrow(b)$ A implicação $(a) \Longrightarrow(b)$ é evidente. Para mostrar $(b) \Longrightarrow(a)$ considere $\mu \in \rho(A)$. Pela identidade do resolvente 2.24, temos

$$
R(\mu, A)=R(\mu, A) R(\lambda, A)(\lambda-\mu)+R(\lambda, A) .
$$

Pela propriedade de ideal de operadores compactos $2.27, R(\mu, A)$ é compacto.

$(a) \Longleftrightarrow(c)$ Seja $\lambda \in \rho(A)$. Como a injeção canônica $i$ é a composição de $(\lambda-A)$ : $[D(A)] \rightarrow X \operatorname{com} R(\lambda, A): X \rightarrow X$ e o operador $R(\lambda, A): X \rightarrow X$ é a composição de $R(\lambda, A): X \rightarrow[D(A)]$ com a injeção canônica $i$, então, pela propriedade de ideal de operadores compactos 2.27, basta mostrar que os operadores $(\lambda-A):[D(A)] \rightarrow X \mathrm{e}$ $R(\lambda, A): X \rightarrow[D(A)]$ são contínuos.

Por um lado

$$
\|(\lambda-A) x\| \leq|\lambda|\|x\|+\|A x\| \leq(|\lambda|+1)\|x\|_{A} .
$$

Por outro lado

$$
\|R(\lambda, A) x\|_{A}=\|R(\lambda, A) x\|+\|A R(\lambda, A) x\| \leq c\|x\|
$$

para algum $c>0$, já que $R(\lambda, A)$ e $A R(\lambda, A)=\lambda R(\lambda, A)-I$ são contínuos.

Em seguida, verificamos algumas propriedades espectrais de operadores com resolvente compacto.

Proposição 2.32. (Propriedades espectrais de operadores com resolvente compacto)

Seja A um operador com resolvente compacto, então

(a) $\sigma(A)=\sigma_{p}(A)$.

(b) $\sigma(A)$ é finito ou existe uma sequência $\left(\lambda_{n}\right)_{n \in \mathbb{N}}$ em $\mathbb{C}$ tal que $\left|\lambda_{n}\right| \rightarrow \infty$ e $\sigma(A)=$ $\left\{\lambda_{n} \in \mathbb{C}: n \in \mathbb{N}\right\}$.

(c) $\operatorname{dim} \operatorname{ker}(\lambda-A)<\infty$, para todo $\lambda \in \mathbb{C}$. 
Demonstração.

(a) Seja $\lambda_{0} \in \rho(A)$. Pelas Proposições 2.29(a) e 2.23 temos

$$
\lambda \in \sigma(A) \Longleftrightarrow \frac{1}{\lambda_{0}-\lambda} \in \sigma\left(R\left(\lambda_{0}, A\right)\right) \Longleftrightarrow \frac{1}{\lambda_{0}-\lambda} \in \sigma_{p}\left(R\left(\lambda_{0}, A\right)\right) \Longleftrightarrow \lambda \in \sigma_{p}(A) .
$$

(b) Segue direto das Proposições 2.29(b) e 2.23.

(c) Seja $\lambda_{0} \in \rho(A)$ e $x \in \operatorname{ker}(\lambda-A)$. Então temos

$$
\begin{aligned}
A x=\lambda x & \Longleftrightarrow\left(\lambda_{0}-A\right) x=\left(\lambda_{0}-\lambda\right) x \\
& \Longleftrightarrow R\left(\lambda_{0}, A\right) x=\left(\lambda_{0}-\lambda\right)^{-1} x \\
& \Longleftrightarrow x \in \operatorname{ker}\left(\left(\lambda_{0}-\lambda\right)^{-1}-R\left(\lambda_{0}, A\right)\right),
\end{aligned}
$$

que é finito, pois $R\left(\lambda_{0}, A\right)$ é compacto.

Proposição 2.33. (Similaridade)

Seja A um operador em $X, Y$ um espaço de Banach sobre $\mathbb{C}, V: X \rightarrow Y$ um isomorfismo e defina o operador $B$ em $Y$ por $B y=V A V^{-1} y$, onde $D(B)=\{y \in Y$ : $\left.V^{-1} y \in D(A)\right\}$. As seguintes afirmações são verdadeiras:

(a) B é fechado se, e somente se, A é fechado.

(b) B tem resolvente compacto se, e somente se, A tem resolvente compacto.

(c) $\rho(B)=\rho(A)$ e $R(\lambda, B)=V R(\lambda, A) V^{-1}$, para todo $\lambda \in \rho(B)$.

Demonstração.

(a) Segue de imediato, já que $V$ e $V^{-1}$ são operadores contínuos.

(b) É consequência direta de (c) e da Proposição 2.27.

(c) Seja $\lambda \in \rho(B)$. Temos

$$
\begin{aligned}
(\lambda-A) & =\left(\lambda-V^{-1} B V\right) \\
& =\left(V^{-1} \lambda V-V^{-1} B V\right) \\
& =V^{-1}(\lambda-B) V .
\end{aligned}
$$


$\operatorname{Logo} \lambda \in \rho(A)$ e $R(\lambda, A)=V^{-1} R(\lambda, B) V$.

\subsection{Operadores não-contínuos em espaços de Hilbert}

Iniciaremos esta seção com a definição de operadores dissipativos. Veremos mais à frente que as possibilidades do conjunto resolvente deste tipo de operador são mais restritas.

Definição 2.34. (Operador dissipativo)

Um operador $A$ em $H$ é dissipativo se $\operatorname{Re}\langle A x, x\rangle \leq 0$, para todo $x \in D(A)$.

Em seguida, obtemos uma definição equivalente para operadores dissipativos.

Proposição 2.35. (Caracterização de operadores dissipativos)

Seja A um operador em H. O operador A é dissipativo se, e somente se,

$$
\|(I-\lambda A) x\| \geq\|x\|
$$

para todo $x \in D(A), \lambda>0$.

Demonstração. Por um lado, se $A$ é dissipativo então, para todo $x \in D(A)$ e para todo $\lambda>0$, temos

$\|(I-\lambda A) x\|\|x\| \geq|\langle(I-\lambda A) x, x\rangle| \geq \operatorname{Re}\langle(I-\lambda A) x, x\rangle=\operatorname{Re}\langle x, x\rangle-\lambda \operatorname{Re}\langle A x, x\rangle \geq\|x\|^{2}$.

Por outro lado, se a desigualdade vale, então

$$
0 \geq\|x\|^{2}-\|(I-\lambda A) x\|^{2}=2 \lambda \operatorname{Re}\langle A x, x\rangle-\lambda^{2}\|A x\|^{2} .
$$

Dividindo por $\lambda$ e fazendo $\lambda \rightarrow 0$, temos

$$
\operatorname{Re}\langle A x, x\rangle \leq 0
$$

para todo $x \in D(A)$.

\section{Corolário 2.36.}

Seja A um operador em $H$ dissipativo, então o operador $(I-A)$ é injetor e o operador $(I-A)^{-1}: i m(I-A) \rightarrow H$ é contínuo. 
Demonstração. A desigualdade

$$
\|(I-A) x\| \geq\|x\|
$$

mostra que se $x \neq 0$, então $(I-A) x \neq 0$. Substituindo $(I-A) x$ por $z$ temos

$$
\left\|(I-A)^{-1} z\right\| \leq\|z\| .
$$

Logo $(I-A)^{-1}$ é contínuo.

Denote $\mathbb{C}_{+}=\{\lambda \in \mathbb{C}: \operatorname{Re}(\lambda)>0\}$ o semiplano complexo com parte real positiva. O próximo resultado revela uma condição para que este semiplano esteja contido no conjunto resolvente de um operador dissipativo.

\section{Proposição 2.37 .}

Sejam A um operador dissipativo em $H$ e $\lambda \in \mathbb{C}_{+}$. Se $(\lambda-A)$ é sobrejetor, então $\mathbb{C}_{+} \subset \rho(A)$ e $\|R(\lambda, A)\| \leq \frac{1}{\operatorname{Re}(\lambda)}$, para todo $\lambda \in \mathbb{C}_{+}$.

Demonstração. Primeiro mostramos que $\lambda \in \rho(A)$. Suponha que o operador $(\lambda-A)$ não é injetor. Então existe $x \in D(A), x \neq 0$, tal que $(\lambda-A) x=0$. Portanto

$$
\operatorname{Re}\langle A x, x\rangle=\operatorname{Re}\langle\lambda x, x\rangle=\operatorname{Re} \lambda\|x\|^{2}>0,
$$

o que contraria a hipótese de que $A$ é dissipativo. Logo $(\lambda-A)$ é injetor e, pela hipótese, sobrejetor, portanto a inversa $(\lambda-A)^{-1}$ existe. Falta mostrar que ela é contínua.

Utilizando que $A$ é dissipativo e a desigualdade de Cauchy-Schwartz, temos

$$
\begin{aligned}
\operatorname{Re} \lambda\|x\|^{2} & =\operatorname{Re}\langle\lambda x, x\rangle \\
& =\operatorname{Re}\langle(\lambda-A) x+A x, x\rangle \\
& =\operatorname{Re}\langle(\lambda-A) x, x\rangle+\operatorname{Re}\langle A x, x\rangle \\
& \leq \operatorname{Re}\langle(\lambda-A) x, x\rangle \\
& \leq\|(\lambda-A) x\|\|x\| .
\end{aligned}
$$

Dividindo por $\|x\|$ e fazendo $y=(\lambda-A) x$ temos

$$
\left\|(\lambda-A)^{-1} y\right\| \leq\|y\| / \operatorname{Re} \lambda .
$$


$\operatorname{Logo}(\lambda-A)^{-1}$ é contínua e para todo $\lambda \in \rho(A) \cap \mathbb{C}_{+}$

$$
\|R(\lambda, A)\| \leq 1 / \operatorname{Re}(\lambda)
$$

Para mostrar que $\mathbb{C}_{+} \subset \rho(A)$ basta provar que $\rho(A) \cap \mathbb{C}_{+}$é aberto e fechado em $\mathbb{C}_{+}$. Como $\rho(A)$ é aberto, então $\rho(A) \cap \mathbb{C}_{+}$é aberto em $\mathbb{C}_{+}$. Agora seja $x_{n}$ uma sequência em $\rho(A) \cap \mathbb{C}_{+}$tal que $x_{n} \rightarrow x \in \mathbb{C}_{+}$. Utilizando a equação (9), aplicamos o Corolário 2.22 e, portanto, $x \in \rho(A)$. Logo $\rho(A) \cap \mathbb{C}_{+}$é fechado em $\mathbb{C}_{+}$. Já que $\mathbb{C}_{+}$é conexo, então $\rho(A) \cap \mathbb{C}_{+}=\mathbb{C}_{+}$.

Definição 2.38. (Operador m-dissipativo)

Um operador $A$ em $H$ é m-dissipativo se é dissipativo e $(I-A)$ é sobrejetor. Se $A$ é um operador m-dissipativo em $H$, então 2.37 implica $\mathbb{C}_{+} \subset \rho(A)$ e $\|R(\lambda, A)\| \leq \frac{1}{\operatorname{Re}(\lambda)}$, para todo $\lambda \in \mathbb{C}_{+}$.

Adiante vamos estabelecer relações entre operadores dissipativos, simétricos e autoadjuntos. Para tanto, vamos definir as noções de adjunta, operador simétrico e operador autoadjunto.

Definição 2.39. (Adjunta)

Seja $A$ um operador em $H$ densamente definido. A adjunta de $A$ é o operador $A^{*}: D\left(A^{*}\right) \subset H \rightarrow H$ com domínio definido por

$$
D\left(A^{*}\right)=\{y \in H: \exists z \in H \text { tal que }\langle A x, y\rangle=\langle x, z\rangle, \forall x \in D(A)\} .
$$

$\mathrm{O}$ valor de $A^{*}$ em $y$ é definido por $A^{*} y=z$. Pela definição, para todo $x \in D(A)$ e para todo $y \in D\left(A^{*}\right)$, temos

$$
\langle A x, y\rangle=\left\langle x, A^{*} y\right\rangle
$$

Definição 2.40. (Operador simétrico e autoadjunto)

Seja $A$ um operador densamente definido em $H$. O operador $A$ é simétrico se $A \subset A^{*}$ e autoadjunto se $A=A^{*}$.

Abaixo seguem diferentes caracterizações para operadores simétricos.

Proposição 2.41. (Caracterizações dos operadores simétricos)

Seja A um operador em $H$. As seguintes afirmações são equivalentes: 
(a) A é simétrico.

(b) $\langle A x, y\rangle=\langle x, A y\rangle$, para todo $x, y \in D(A)$.

(c) $\langle A x, x\rangle \in \mathbb{R}$, para todo $x \in D(A)$.

(d) $i A$ e $-i A$ são dissipativos.

Demonstração.

$(a) \Longleftrightarrow(b)$ Pela definição da adjunta, $D(A) \subset D\left(A^{*}\right)$ e $A x=A^{*} x$ para todo $x \in D(A)$ se, e somente se, para todo $x, y \in D(A),\langle A x, y\rangle=\langle x, A y\rangle$.

$(b) \Longrightarrow \quad(c)$ Para todo $x \in D(A)$ temos $\langle A x, x\rangle=\langle x, A x\rangle=\overline{\langle A x, x\rangle}$. Logo $\langle A x, x\rangle \in \mathbb{R}$.

$(c) \Longrightarrow(b)$ Seja $x, y \in D(A)$. Utilizando a identidade de polarização, temos

$$
\begin{aligned}
\overline{\langle A x, y\rangle} & =\overline{\frac{1}{4} \sum_{j=0}^{3} i^{j}\left\langle A\left(x+i^{j} y\right), x+i^{j} y\right\rangle} \\
& =\frac{1}{4} \sum_{j=0}^{3}(-i)^{j}\left\langle A\left(x+i^{j} y\right), x+i^{j} y\right\rangle \\
& =\frac{1}{4} \sum_{j=0}^{3}(-i)^{j}\left\langle A\left((-i)^{j} x+y\right),(-i)^{j} x+y\right\rangle \\
& =\langle A y, x\rangle .
\end{aligned}
$$

Logo, $\langle A x, y\rangle=\overline{\langle A y, x\rangle}=\langle x, A y\rangle$.

$(c) \Longrightarrow(d) \operatorname{Re}\langle \pm i A x, x\rangle=\operatorname{Re} \pm i\langle A x, x\rangle=0$

$(d) \Longrightarrow(c)$ Pela hipótese, $\pm \operatorname{Re} i\langle A x, x\rangle \leq 0$, então $\operatorname{Re} i\langle A x, x\rangle=0 . \operatorname{Logo}\langle A x, x\rangle \in$ $\mathbb{R}$.

\section{Proposição 2.42.}

Se A é um operador simétrico em $H$, existem 4 possibilidades:

(a) $\sigma(A)=\{\lambda \in \mathbb{C}: \operatorname{Im}(\lambda) \geq 0\}$.

(b) $\sigma(A)=\{\lambda \in \mathbb{C}: \operatorname{Im}(\lambda) \leq 0\}$. 
(c) $\sigma(A)=\mathbb{C}$.

(d) $\sigma(A) \subset \mathbb{R}$.

Demonstração.

É consequência direta das Proposições 2.41(d), 2.37 e do fato que $\sigma(A)$ é fechado.

Agora estabelecemos algumas propriedades úteis que serão utilizadas posteriormente.

\section{Proposição 2.43.}

Seja A um operador em $H$. As seguintes afirmações são verdadeiras:

(a) $A^{*}$ é um operador fechado.

(b) A é autoadjunto (simétrico) $\Longleftrightarrow(I-A)$ é autoadjunto (simétrico).

(c) $(\operatorname{im}(A))^{\perp}=\operatorname{ker}\left(A^{*}\right)$.

(d) A é simétrico e sobrejetor $\Longrightarrow A$ é autoadjunto.

Demonstração.

(a) Equipando o espaço $H \times H$ com o produto interno $\langle(x, y),(z, w)\rangle:=\langle x, z\rangle+\langle y, w\rangle$, note que pela definição da adjunta temos

$$
\begin{aligned}
G\left(A^{*}\right) & =\{(y, z) \in H \times H \mid \forall x \in D(A),\langle A x, y\rangle=\langle x, z\rangle\} \\
& =\{(y, z) \in H \times H \mid \forall(x, A x) \in G(A),\langle(A x,-x),(y, z)\rangle=0\} \\
& =\{(A x,-x) \in H \times H \mid x \in D(A)\}^{\perp} .
\end{aligned}
$$

Como o complemento de um conjunto é sempre fechado, segue que $G\left(A^{*}\right)$ é fechado. (b) Sejam $x, y \in D(A)$. Por um lado, se $A$ é simétrico, então

$$
\langle(I-A) x, y\rangle=\langle x, y\rangle-\langle A x, y\rangle=\langle x, y\rangle-\langle x, A y\rangle=\langle x,(I-A) y\rangle .
$$

Logo $(I-A)$ é simétrico. Sejam $x \in D(A)$ e $y \in D(I-A)^{*}$. Se $A$ é autoadjunto, então

$$
\begin{aligned}
\langle A x, y\rangle & =\langle(I-(I-A)) x, y\rangle \\
& =\langle x, y\rangle-\langle(I-A) x, y\rangle \\
& =\langle x, y\rangle-\left\langle x,(I-A)^{*} y\right\rangle \\
& =\left\langle x,\left(I-(I-A)^{*}\right) y\right\rangle .
\end{aligned}
$$


Logo, pela definição de adjunta, temos

$$
I-(I-A)^{*} \subset A^{*}=A
$$

que implica

$$
(I-A)^{*} \subset(I-A) \text {. }
$$

Logo $(I-A)$ é autoadjunto. Por outro lado, se $(I-A)$ é autoadjunto (simétrico), usando o resultado anterior, então $I-(I-A)=A$ é autoadjunto (simétrico).

(c) Temos $y \in(\operatorname{im}(A))^{\perp} \Longleftrightarrow\langle y, A x\rangle=0, \forall x \in D(A) \Longleftrightarrow(y, 0) \in G\left(A^{*}\right) \Longleftrightarrow$ $y \in \operatorname{ker}\left(A^{*}\right)$.

(d) Seja $y \in D\left(A^{*}\right)$. Como $A$ é sobrejetor, então existe $x \in D(A)$ tal que $A x=A^{*} y$. Pela definição da adjunta e pelo fato de $A$ ser simétrico, para todo $z \in D(A)$, temos

$$
\langle A z, y\rangle=\left\langle z, A^{*} y\right\rangle=\langle z, A x\rangle=\langle A z, x\rangle
$$

Como $A$ é sobrejetora, isso significa que para todo $w \in H$

$$
\langle w, y\rangle=\langle w, x\rangle
$$

$\operatorname{Logo} y=x \in D(A)$.

Por último, obtemos uma equivalência entre operadores simétricos e m-dissipativos e operadores autoadjuntos e dissipativos.

\section{Proposição 2.44 .}

Um operador $A$ em $H$ é autoadjunto e dissipativo se, e somente se, é m-dissipativo e simétrico. Neste caso $\sigma(A) \subset(-\infty, 0]$.

Demonstração. ( $\Longrightarrow)$ Basta mostrar que $\operatorname{im}(I-A)=H$. Para isso, verificamos que a imagem $\operatorname{im}(I-A)$ é fechada e densa em $\mathrm{H}$.

De fato, pela Proposições 2.43(c), 2.43(b) e pelo Corolário 2.36 temos

$$
\operatorname{im}(I-A)^{\perp}=\operatorname{ker}\left((I-A)^{*}\right)=\operatorname{ker}(I-A)=\{0\}
$$

Logo a imagem $\operatorname{im}(I-A)$ é densa em $\mathrm{H}$.

A imagem $\operatorname{im}(I-A)$ também é fechada. De fato, seja $\left(y_{n}\right)_{n \in \mathbb{N}}$ uma sequência em $\operatorname{im}(I-A)$ 
tal que $y_{n} \rightarrow y \in H$ e $\left(x_{n}\right)_{n \in \mathbb{N}}$ a sequência em $D(A)$ tal que $(I-A) x_{n}=y_{n}$, para todo $n \in \mathbb{N}$. Mostraremos que $y \in \operatorname{im}(I-A)$. Como $(I-A)$ é dissipativo, utilizando a Equação (8), temos para todo $n, m \in \mathbb{N}$

$$
\left\|x_{n}-x_{m}\right\|=\left\|(I-A)^{-1}\left(y_{n}-y_{m}\right)\right\| \leq\left\|y_{n}-y_{m}\right\|
$$

Fazendo $n, m \rightarrow \infty$, então $\left\|y_{n}-y_{m}\right\| \rightarrow 0$, logo $\left\|x_{n}-x_{m}\right\| \rightarrow 0$ e, portanto, existe $x \in H$ tal que $x_{n} \rightarrow x$, pois $H$ é completo. O operador $A$ é fechado pois é autoadjunto (2.43), $\log 0 x \in D(A)$ e $(I-A) x=y \in \operatorname{im}(I-A)$.

$(\Longleftarrow)$ Pela Proposição 2.43, temos que o operador $(I-A)$ é simétrico e sobrejetor (pela hipótese), logo é autoadjunto. Por fim, utilizando novamente a Proposição 2.43(b), o operador $A$ é autoadjunto.

Em todo caso, como $A$ é m-dissipativo, então $\mathbb{C}_{+} \subset \rho(A)$. Logo $(1+i-A)$ e $(1-i-A)$ são sobrejetores. Multiplicando por $-i$ e $i$ respectivamente temos que $(1-i+i A)$ e $(1+i-i A)$ são sobrejetores. Como $A$ é simétrico, então $i A$ e $-i A$ são dissipativos (Proposição 2.41). Logo, pela Proposição 2.37, $\mathbb{C}_{+} \subset \rho(-i A)$ e $\mathbb{C}_{+} \subset \rho(i A)$. Então $\mathbb{C} \backslash(-\infty, \infty) \subset \rho(A)$. Juntando com o fato que $\mathbb{C}_{+} \subset \rho(A)$, então $\mathbb{C} \backslash(-\infty, 0] \subset \rho(A)$.

\subsection{Operadores de multiplicação}

Os operadores de multiplicação e, em particular, os operadores diagonais são exemplos de operadores em geral não-contínuos, que possuem uma estrutura mais simples e fácil de analisar. Na próxima seção, veremos sob quais condições um operador é equivalente a um operador de multiplicação e utilizaremos este resultado com o operador Laplaciano.

\section{Definição 2.45 .}

Sejam $\Omega \subset \mathbb{R}^{n}$ um aberto e $a: \Omega \rightarrow \mathbb{C}$ uma função mensurável. O operador de multiplicação $M_{a}$ em $L^{2}(\Omega)$ é definido por

$$
\left(M_{a} f\right)(x):=a(x) f(x),
$$

onde

$$
D\left(M_{a}\right):=\left\{f \in L^{2}(\Omega): M_{a} f \in L^{2}(\Omega)\right\} .
$$


Proposição 2.46. (Propriedades dos operadores de multiplicação)

(a) $M_{a}$ é um operador fechado.

(b) $M_{a}$ é densamente definido.

(c) $M_{a}$ é contínuo $\Longleftrightarrow a \in L^{\infty}\left(\mathbb{R}^{n}\right)$. Nesse caso $\left\|M_{a}\right\|=\|a\|_{L^{\infty}\left(\mathbb{R}^{n}\right)}$.

(d) $\lambda \in \rho\left(M_{a}\right) \Longrightarrow R\left(\lambda, M_{a}\right)=M_{\frac{1}{\lambda-a}}$.

(e) A imagem de a é quase-sempre real $\Longrightarrow M_{a}$ é autoadjunto.

Demonstração.

(a) Seja $\left(f_{m}\right)_{m \in \mathbb{N}}$ uma sequência em $G\left(M_{a}\right)$ tal que $f_{m} \rightarrow f \in L^{2}(\Omega)$ e $\left(a f_{m}\right) \rightarrow$ $g \in L^{2}(\Omega)$. Tomando uma subsequência caso necessário, podemos supor $f_{m} \rightarrow f$ pontualmente quase-sempre. Mas então $a f_{m} \rightarrow a f$ pontualmente quase-sempre. Logo $f \in D\left(M_{a}\right)$ e $g=a f$.

(b) Seja $f \in L^{2}(\Omega)$ e defina $f_{m}=1_{A_{m}} f$, onde $A_{m}:=\{x \in \Omega:|a(x)| \leq m\}$. Note que $f_{m} \in L^{2}(\Omega)$ e $f_{m} \rightarrow f$ em $L^{2}(\Omega)$ pelo Teorema da Convergência Dominada. Para todo $x \in \Omega,\left|a(x) f_{m}(x)\right| \leq m|f(x)|, \operatorname{logo} M_{a} f_{m} \in L^{2}(\Omega)$ e portanto $f_{m} \in D\left(M_{a}\right)$.

(c) $(\Longrightarrow)$ Seja $A \subset\left\{x \in \Omega:|a(x)|>\left\|M_{a}\right\|\right\}$ tal que $0<\mu(A)<\infty$. Então

$$
\mu(A)\left\|M_{a}\right\|^{2}<\int_{A}|a|^{2}=\left\|M_{a}\left(\chi_{A}\right)\right\|_{2}^{2} \leq \mu(A)\left\|M_{a}\right\|^{2} .
$$

O que leva a contradição $\left\|M_{a}\right\|^{2}<\left\|M_{a}\right\|^{2}$. Logo $a \in L^{\infty}(X)$ e $\|a\|_{\infty} \leq\left\|M_{a}\right\|$.

$$
(\Longleftarrow)
$$

$$
\sup _{\|f\| \leq 1} \int_{X}\left|M_{a} f\right|^{2} \leq\|a\|_{\infty}^{2} .
$$

Logo $M_{a}$ é limitado e $\left\|M_{a}\right\| \leq\|a\|_{\infty}$.

(d) Seja $\lambda \in \rho\left(M_{a}\right)$ e $f \in L^{2}(\Omega)$, então

$$
\left(\left(\lambda I-M_{a}\right) f\right)(x)=(\lambda-a(x)) f(x) .
$$


Logo

$$
\begin{aligned}
\left(R\left(\lambda, M_{a}\right) f\right)(x) & =\left(\left(\lambda I-M_{a}\right)^{-1} f\right)(x) \\
& =(\lambda-a(x))^{-1} f(x) \\
& =\left(M_{\frac{1}{\lambda-a}} f\right)(x) .
\end{aligned}
$$

(e) Seja $f, g \in D\left(M_{a}\right)$, então

$$
\left\langle M_{a} f, g\right\rangle=\int_{\Omega} a(x) f(x) \overline{g(x)} d x=\int_{\Omega} f(x) \overline{a(x) g(x)} d x=\left\langle f, M_{a} g\right\rangle .
$$

Logo $M_{a}$ é simétrico. Agora suponha $f \in D\left(M_{a}^{*}\right)$. Então existe $h \in L^{2}(\Omega)$ tal que, para todo $g \in D\left(M_{a}\right)$, vale

$$
\left\langle M_{a} g, f\right\rangle=\int_{\Omega} a(x) g(x) \overline{f(x)} d x=\int_{\Omega} g(x) \overline{h(x)} d x=\langle g, h\rangle .
$$

Logo temos

$$
\int_{\Omega} g(x)(a(x) \overline{f(x)}-\overline{h(x)}) d x=0 .
$$

Portanto, $\bar{a} f=a f=h \in L^{2}(\Omega)$ e $f \in D\left(M_{a}\right)$.

Se na definição dos operadores de multiplicação substituirmos o espaço $\Omega \subset \mathbb{R}^{n}$ com a medida de Lebesgue pelo espaço $\mathbb{N}$ com a medida de contagem obtemos os operadores diagonais.

Antes de definir os operadores diagonais vamos lembrar que

$$
\ell^{2}:=\left\{\left(x_{m}\right)_{m \in \mathbb{N}} \text { em } \mathbb{C}: \sum_{m=1}^{\infty}\left|x_{m}\right|^{2}<\infty\right\}
$$

é um espaço de Hilbert separável sobre $\mathbb{C}$ com relação ao produto interno $\langle x, y\rangle=$ $\sum_{m=1}^{\infty} x_{m} \bar{y}_{m}$

$$
\ell^{\infty}:=\left\{\alpha=\left(\alpha_{m}\right)_{m \in \mathbb{N}} \text { em } \mathbb{C}: \sup _{m \in \mathbb{N}}\left|\alpha_{m}\right|<\infty\right\}
$$

é um espaço de Banach com relação a norma $\|\alpha\|_{\infty}=\sup _{m \in \mathbb{N}}\left|\alpha_{m}\right|$ e

$$
c_{0}:=\left\{\alpha=\left(\alpha_{m}\right)_{m \in \mathbb{N}} \text { em } \mathbb{C}: \alpha_{m} \rightarrow 0\right\}
$$


é um subespaço fechado de $\ell^{\infty}$.

\section{Definição 2.47.}

Seja $\alpha=\left(\alpha_{m}\right)_{m \in \mathbb{N}}$ uma sequência em $\mathbb{C}$. O operador diagonal $M_{\alpha}$ em $\ell^{2}$ é definido por

$$
M_{\alpha} x=\left(\alpha_{m} x_{m}\right)_{m \in \mathbb{N}}
$$

onde

$$
D\left(M_{\alpha}\right)=\left\{x \in \ell^{2}:\left(\alpha_{m} x_{m}\right)_{m \in \mathbb{N}} \in \ell^{2}\right\}
$$

Proposição 2.48. (Propriedades de operadores diagonais)

(a) $\alpha \in \ell^{\infty} \Longrightarrow M_{\alpha} \in \mathcal{L}\left(\ell^{2}\right) e\left\|M_{\alpha}\right\|=\|\alpha\|_{\infty}$;

(b) $\alpha \in c_{0} \Longrightarrow M_{\alpha} \in \mathcal{K}\left(\ell^{2}\right)$;

(c) $\left|\alpha_{m}\right| \rightarrow \infty \Longrightarrow M_{\alpha}$ tem resolvente compacto;

(d) $\alpha=\left(\alpha_{m}\right)_{m \in \mathbb{N}}$ é uma sequência em $\mathbb{R} \Longrightarrow M_{\alpha}$ é autoadjunto.

Demonstração.

(a) Seja $x \in \ell^{2}$. Então

$$
\left\|M_{\alpha} x\right\|_{2}^{2}=\sum_{m=1}^{\infty}\left|\alpha_{m} x_{m}\right|^{2} \leq \sum_{m=1}^{\infty}\|\alpha\|_{\infty}^{2}\left|x_{m}\right|^{2}=\|\alpha\|_{\infty}^{2}\|x\|_{2}^{2}
$$

Logo $M_{\alpha} x \in \ell^{2}, M_{\alpha} \in \mathcal{L}\left(\ell^{2}\right)$ e $\left\|M_{\alpha}\right\| \leq\|\alpha\|_{\infty}$. Por fim, seja $c<\|\alpha\|_{\infty}$. Então existe $m_{0} \in \mathbb{N}$ tal que $\alpha_{m_{0}}>c$. Seja $x \in \ell^{2}$ tal que $x_{m}=1$ se $m=m_{0}$ e $x_{m}=0$, caso contrário. Temos $\|x\|_{2}=1$ e $\left\|M_{\alpha} x\right\|_{2}=\alpha_{m_{0}}>c$. Logo $\left\|M_{\alpha}\right\|=\|\alpha\|_{\infty}$.

(b) Seja $\left(T_{j}\right)_{j \in \mathbb{N}}$ a sequência de operadores em $\ell^{2}$ definida por

$$
\left(T_{j} x\right)_{m}= \begin{cases}\alpha_{m} x_{m} & , \text { se } 1 \leq m \leq j \\ 0 & , \text { se } m>j\end{cases}
$$

Logo

$$
\left(\left(M_{\alpha}-T_{j}\right) x\right)_{m}= \begin{cases}0 & , \text { se } 1 \leq m \leq j \\ \alpha_{m} x_{m} & , \text { se } m>j\end{cases}
$$


Para todo $j \in \mathbb{N}$, o operador $T_{j}$ tem posto finito e

$$
\lim _{j \rightarrow \infty}\left\|M_{\alpha}-T_{j}\right\|=\lim _{j \rightarrow \infty} \alpha_{j}=0
$$

Logo $M_{\alpha}$ é compacto.

(c) Seja $\lambda \in \rho\left(M_{\alpha}\right)$, então

$$
R\left(M_{\alpha}, \lambda\right) x=\left(\lambda I-M_{\alpha}\right)^{-1} x=\left(\left(\lambda-\alpha_{m}\right)^{-1} x_{m}\right)_{m \in \mathbb{N}} .
$$

Se $\left|\alpha_{m}\right| \rightarrow \infty$, então $\left(\lambda-\alpha_{m}\right)^{-1} \rightarrow 0$. Logo, pelo item (b), $R\left(M_{\alpha}, \lambda\right)$ é compacto.

(d) Seja $x, y \in \ell^{2}$, então

$$
\left\langle M_{\alpha} x, y\right\rangle=\sum_{m=1}^{\infty} \alpha_{m} x_{m} \overline{y_{m}}=\sum_{m=1}^{\infty} x_{m} \overline{\alpha_{m} y_{m}}=\left\langle x, M_{\alpha} y\right\rangle
$$

Logo $M_{\alpha}$ é simétrico. Agora suponha $x \in D\left(M_{a}^{*}\right)$. Então existe $z \in \ell^{2}$ tal que, para todo $y \in D\left(M_{\alpha}\right)$, vale

$$
\left\langle M_{\alpha} y, x\right\rangle=\sum_{m=1}^{\infty} \alpha_{m} y_{m} \overline{x_{m}}=\sum_{m=1}^{\infty} y_{m} \overline{z_{m}}=\langle y, z\rangle .
$$

Logo, para todo $m \in \mathbb{N}$, temos

$$
y_{m}\left(\alpha_{m} \overline{x_{m}}-\overline{z_{m}}\right)=0 .
$$

Portanto, $\left(\overline{\alpha_{m}} x_{m}\right)_{m \in \mathbb{N}}=\left(\alpha_{m} x_{m}\right)_{m \in \mathbb{N}}=\left(z_{m}\right)_{m \in \mathbb{N}} \in \ell^{2}$ e $x \in D\left(M_{\alpha}\right)$.

\subsection{Teorema espectral}

O resultado desta seção é o objetivo principal do capítulo. O Teorema Espectral é extremamente útil, pois permite representar certos operadores como operadores diagonais. Fazemos a demonstração do Teorema Espectral para operadores autoadjuntos, dissipativos e com resolvente compacto. O caso mais geral para operadores autoadjuntos e dissipativos é enunciado e sua demonstração pode ser consultada em [25].

Primeiro enunciamos o Teorema espectral para operadores compactos e autoadjuntos. 
Proposição 2.49. (Teorema espectral para operadores compactos autoadjuntos)

Seja $A: H \rightarrow H$ um operador compacto e autoadjunto. Então existe $N \subset \mathbb{N}$ e $H$ possui um sistema ortonormal $\left(e_{j}\right)_{j \in N}$ de autovetores de A com correspondente autovalores $\left(\lambda_{j}\right)_{j \in N}$ em $\mathbb{R} \backslash\{0\}$, tal que $\lim _{j \rightarrow \infty} \lambda_{j}=0$ e, para todo $x \in D(A)$, temos

$$
A x=\sum_{j \in N} \lambda_{j}\left\langle x, e_{j}\right\rangle e_{j}
$$

Teorema 2.50. (Teorema Espectral para operadores simétricos, m-dissipativos e com resolvente compacto)

Seja A um operador em $H$ simétrico, m-dissipativo e com resolvente compacto. Então $H$ possui uma base ortonormal $\left\{e_{n}: n \in \mathbb{N}\right\} \subset D(A)$ e, para todo $n \in \mathbb{N}$, existe $\lambda_{n} \leq 0$ tal que $A e_{n}=\lambda_{n} e_{n}$ e $\lambda_{n} \rightarrow-\infty$. Além disso A é dado por

$$
A x=\sum_{n=1}^{\infty} \lambda_{n}\left\langle x, e_{n}\right\rangle e_{n},
$$

onde

$$
D(A)=\left\{x \in H:\left(\lambda_{n}\left\langle x, e_{n}\right\rangle\right)_{n \in \mathbb{N}} \in \ell^{2}\right\}
$$

Demonstração.

Como $A$ é simétrico e m-dissipativo, então $(0, \infty) \subset \rho(A)$. Seja $\mu \in(0, \infty) \subset \rho(A)$. Como $A$ é simétrico, então para todo $x, y \in H$, temos

$$
\begin{aligned}
\langle R(\mu, A) x, y\rangle & =\langle R(\mu, A) x,(\mu-A) R(\mu, A) y\rangle \\
& =\langle(\mu-A) R(\mu, A) x, R(\mu, A) y\rangle \\
& =\langle x, R(\mu, A) y\rangle .
\end{aligned}
$$

Logo $R(\mu, A)$ é simétrico. Como $R(\mu, A)$ é limitado, então $R(\mu, A)$ é autoadjunto.

Pelo Teorema Espectral para operadores compactos e autoadjuntos, existe uma base ortonormal $\left\{e_{n}: n \in \mathbb{N}\right\}$ de $H$ e uma sequência $\left(\alpha_{n}\right)_{n \in \mathbb{N}}$ em $\mathbb{R}$ tal que $R(\mu, A) e_{n}=\alpha_{n} e_{n} \in$ $D(A)$. Logo $e_{n} \in D(A)$ e $e_{n}=\alpha_{n}(\mu-A) e_{n}$. Como $R(\mu, A)$ é injetor, então $\alpha_{n} \neq 0$, para todo $n \in \mathbb{N}$. Fazendo $\lambda_{n}=\left(\mu-\alpha_{n}{ }^{-1}\right)$ temos $A e_{n}=\lambda_{n} e_{n}$, para todo $n \in \mathbb{N}$. Além do mais, já que $A$ é dissipativo, $\lambda_{n}=\left\langle A e_{n}, e_{n}\right\rangle \leq 0$, para todo $n \in \mathbb{N}$. Como $\left|\alpha_{n}\right| \rightarrow 0$, então $\lambda_{n} \rightarrow-\infty$. 
Seja $x \in D(A)$, então

$$
\sum_{n=1}^{\infty}\left|\lambda_{n}\left\langle x, e_{n}\right\rangle\right|^{2}=\sum_{n=1}^{\infty}\left|\left\langle x, A e_{n}\right\rangle\right|^{2}=\sum_{n=1}^{\infty}\left|\left\langle A x, e_{n}\right\rangle\right|^{2}=\|A x\|^{2} \leq \infty
$$

$\operatorname{Logo}\left(\lambda_{n}\left\langle x, e_{n}\right\rangle\right)_{n \in \mathbb{N}} \in \ell^{2} \mathrm{e}$

$$
A x=\sum_{n=1}^{\infty}\left\langle A x, e_{n}\right\rangle e_{n}=\sum_{n=1}^{\infty} \lambda_{n}\left\langle x, e_{n}\right\rangle e_{n} .
$$

Reciprocamente, seja $x \in H$ tal que $\left(\lambda_{n}\left\langle x, e_{n}\right\rangle\right)_{n \in \mathbb{N}} \in \ell^{2}$. Defina, para todo $m \in \mathrm{N}$

$$
\begin{aligned}
& x_{m}=\sum_{n=1}^{m}\left\langle x, e_{n}\right\rangle e_{n} \\
& y_{m}=A x_{m}=\sum_{n=1}^{m} \lambda_{n}\left\langle x, e_{n}\right\rangle e_{n} .
\end{aligned}
$$

Logo $\left(x_{m}\right)_{m \in \mathbb{N}}$ é uma sequência em $D(A), x_{m} \rightarrow x$ e $y_{m} \rightarrow y \in H$. Como $A$ é fechado, então $x \in D(A)$ e $A x=y=\sum_{n=1}^{\infty} \lambda_{n}\left\langle x, e_{n}\right\rangle e_{n}$.

\section{Corolário 2.51.}

Nas condições do Teorema 2.50, seja $U: H \rightarrow \ell^{2}$ o operador unitário definido por $U x=\left(\left\langle x, e_{n}\right\rangle\right)_{n \in \mathbb{N}}$, então $U A U^{-1}=M_{\lambda}$. Ou seja, A é unitariamente equivalente ao operador diagonal $M_{\lambda}$, onde $\lambda=\left(\lambda_{j}\right)_{j \in \mathbb{N}}$.

Por fim, enunciamos a seguir a generalização do teorema anterior.

Teorema 2.52. (Teorema Espectral para operadores simétricos e m-dissipativos)

Seja $A$ um operador em $H$ autoadjunto e dissipativo. Então A unitariamente equivalente a um operador de multiplicação. Isto é, existe um espaço de medida finito $Y$, uma função mensurável $a: Y \rightarrow(-\infty, 0)$ e um operador unitário $U: H \rightarrow L^{2}(Y)$, tal que

$$
U A U^{-1}=M_{a}
$$




\section{Semigrupos}

Neste capítulo fazemos uma breve introdução à teoria dos semigrupos, desenvolvendo os aspectos utilizados na demonstração da Lei de Weyl. Foram utilizados os textos [2] e [16] como principais referências.

\subsection{Semigrupos fortemente contínuos}

Semigrupos fortemente contínuos podem ser vistos como uma generalização da função exponencial. Veremos que eles fornecem as soluções de equações diferenciais ordinárias lineares com coeficientes constantes e com valores em espaços de Banach.

Definição 3.1. (Semigrupo fortemente contínuo)

Um semigrupo fortemente contínuo ou um $C_{0}$-semigrupo em $X$ é uma função $T:[0, \infty) \rightarrow \mathcal{L}(X)$ que satisfaz as seguintes condições:

(a) $T(0)=I$,

(b) $T(t+s)=T(t) T(s)$, para todo $t, s \geq 0$,

(c) $T_{x}:[0, \infty) \ni t \mapsto T(t) x \in X$ é contínua, para todo $x \in X$.

Segue de (b) que $T(t) T(s)=T(s) T(t)$, para todo $t, s \geq 0$.

Definição 3.2. (Gerador)

O gerador $A$ do $C_{0}$-semigrupo $T$ é um operador em $X$ definido por

$$
A x=\lim _{h \rightarrow 0^{+}} \frac{(T(h)-I) x}{h},
$$

onde

$$
D(A)=\left\{x \in X: \lim _{h \rightarrow 0^{+}} \frac{(T(h)-I) x}{h} \text { existe em } X\right\} .
$$

Temos as seguintes propriedades básicas.

\section{Proposição 3.3.}

Seja $A$ o gerador do $C_{0}$-semigrupo $T$ em $X$, então $T(t) x \in D(A)$ e $A T(t) x=T(t) A x$, para todo $x \in D(A)$ e $t \geq 0$. 
Demonstração.

Seja $x \in D(A)$ e $t \geq 0$. Temos

$$
\lim _{h \rightarrow 0^{+}} \frac{(T(h)-I) T(t) x}{h}=T(t) \lim _{h \rightarrow 0^{+}} \frac{(T(h)-I) x}{h}=T(t) A x .
$$

Isso mostra que a derivada lateral pela direita de $T(t)$ em $x \in D(A)$ no ponto zero é $T(t) A x$.

\section{Proposição 3.4.}

Para todo $C_{0}$-semigrupo $T$, existem constantes $M \geq 1 e \omega \in \mathbb{R}$ tais que, para todo $t \geq 0$

$$
\|T(t)\| \leq M e^{\omega t}
$$

Demonstração.

Pela definição de $C_{0}$-semigrupo, para todo $x \in X$ a função $T_{x}: t \mapsto T(t) x$ é contínua em $[0, \infty)$. Portanto, para todo $x \in X$, temos

$$
\sup _{t \in[0,1]}\left\|T_{x}(t)\right\|<\infty
$$

Logo, pelo Teorema de Banach-Steinhaus 2.8, temos

$$
\sup _{t \in[0,1]}\|T(t)\|=M<\infty .
$$

Seja $t=j+s \geq 0$ tal que $j \in \mathbb{N}$ e $s \in[0,1)$, então

$$
\|T(t)\|=\left\|T(1)^{j} T(s)\right\| \leq M^{j+1}=M e^{j \log M} .
$$

Seja $\omega=\log M$. Se $\omega \geq 0$ então

$$
\|T(t)\| \leq M e^{j \omega} \leq M e^{\omega t}
$$

Se $\omega<0$ então

$$
\|T(t)\| \leq M e^{j \omega} \leq M e^{-\omega} e^{(j+1) \omega} \leq M e^{-\omega} e^{\omega t},
$$

como queríamos.

Definição 3.5. (Limite de crescimento) 
O limite de crescimento $\omega_{0}$ de um $C_{0}$-semigrupo $T$ é definido por

$$
\omega_{0}:=\inf \left\{\omega \in \mathbb{R}: \exists M \geq 1 \text { tal que }\|T(t)\| \leq M e^{\omega t}, \forall t \geq 0\right\}
$$

O resultado abaixo mostra que $C_{0}$-semigrupos descrevem as soluções para os chamados problemas abstratos de Cauchy.

\section{Proposição 3.6.}

Seja $A$ o gerador do $C_{0}$-semigrupo $T$ em $X$ e $x \in D(A)$. Então $u:[0, \infty) \rightarrow X$, dado por $u(t)=T(t) x$, é a única solução do problema de valor inicial:

$$
\left\{\begin{array}{l}
u \in C^{1}([0, \infty), X), u(t) \in D(A), t \in[0, \infty) \\
u^{\prime}(t)=A u(t), t \in[0, \infty) \\
u(0)=x
\end{array}\right.
$$

Demonstração.

Seja $t \geq 0$. Pela Proposição 3.4, $T$ é limitado em $[0, t]$. A derivada lateral pela esquerda de $T(t) x$ também é $T(t) A x$. De fato

$$
\begin{aligned}
\lim _{h \rightarrow 0^{-}} \frac{T(t+h) x-T(t) x}{h} & =\lim _{h \rightarrow 0^{+}} \frac{T(t) x-T(t-h) x}{h} \\
& =\lim _{h \rightarrow 0^{+}} T(t-h) \frac{T(h) x-x}{h} \\
& =\lim _{h \rightarrow 0^{+}}\left[T(t-h)\left(\frac{T(h) x-x}{h}-A x\right)+T(t-h) A x\right] \\
& =T(t) A x .
\end{aligned}
$$

Logo $u$ é solução do problema (10). Seja $v$ uma outra solução, $t \geq 0$ e $w:[0, t] \rightarrow X$, definido por $w(s)=T(t-s) v(s)$. Uma simples repetição do argumento usual mostra que vale a regra da derivada do produto $(T(t) v(t))^{\prime}=T^{\prime}(t) v(t)+T(t) v^{\prime}(t)$. Logo temos

$$
\begin{aligned}
w^{\prime}(s) & =-A(T(t-s) v(s))+T(t-s) v^{\prime}(s) \\
& =-T(t-s) A v(s)+T(t-s) A v(s)=0 .
\end{aligned}
$$

Portanto, $w$ é constante e $T(t) x=w(0)=w(t)=v(t)$.

O próximo lema será utilizado para mostrar que o gerador de um $C_{0}$-semigrupo é 
fechado.

\section{Lema 3.7.}

Seja $A$ o gerador do $C_{0}$-semigrupo $T$ em $X$ e $x, y \in X$. Então

$$
x \in D(A) \text { e } A x=y \Longleftrightarrow \int_{0}^{t} T(s) y d s=T(t) x-x .
$$

Demonstração.

$(\Longleftarrow)$ Pela hipótese temos

$$
\lim _{t \rightarrow 0^{+}} \frac{T(t) x-x}{t}=\lim _{t \rightarrow 0^{+}} \frac{1}{t} \int_{0}^{t} T(s) y d s=y
$$

$\log 0 x \in D(A)$ e $A x=y$.

( $\Longrightarrow$ ) Seja $x \in D(A)$. Assim $T_{x}$ é solução de (10). Pelo Teorema Fundamental do Cálculo, temos

$$
T(t) x-x=\int_{0}^{t} T^{\prime}(s) x d s=\int_{0}^{t} T(s) A x d s
$$

como desejado.

\section{Proposição 3.8 .}

$O$ gerador $A$ de um $C_{0}$-semigrupo $T$ em $X$ é fechado.

\section{Demonstração.}

Seja $\left(x_{n}\right)_{n \in \mathbb{N}}$ uma sequência em $D(A)$ tal que $x_{n} \rightarrow x, A x_{n}=y_{n} \rightarrow y$. Temos

$$
\int_{0}^{t} T(s) y_{n} d s=T(t) x_{n}-x_{n}
$$

para todo $n \in \mathbb{N}$. Fazendo $n \rightarrow \infty$ temos

$$
\int_{0}^{t} T(s) y d s=T(t) x-x .
$$

Logo $x \in D(A)$ e $A x=y$.

Para mostrar que o gerador de um $C_{0}$-semigrupo é densamente definido, precisamos do seguinte lema. 


\section{Lema 3.9.}

Seja $T$ um $C_{0}$-semigrupo com gerador $A$. Então $\int_{0}^{t} T(s) x d s \in D(A)$ e $A \int_{0}^{t} T(s) x d s=$ $T(t) x-x$, para todo $x \in X$ e $t \geq 0$.

Demonstração.

Utilizando a definição de gerador, temos

$$
\begin{aligned}
\frac{1}{h}\left[T(h) \int_{0}^{t} T(s) x d s-\int_{0}^{t} T(s) x d s\right] & =\frac{1}{h}\left[\int_{0}^{t} T(s+h) x d s-\int_{0}^{t} T(s) x d s\right] \\
& =\frac{1}{h}\left[\int_{h}^{t+h} T(s) x d s-\int_{0}^{t} T(s) x d s\right] \\
& =\frac{1}{h}\left[\int_{t}^{t+h} T(s) x d s-\int_{0}^{h} T(s) x d s\right]
\end{aligned}
$$

que converge para $T(t) x-x$, quando $h \rightarrow 0^{+}$.

\section{Proposição 3.10 .}

Seja $A$ o gerador do $C_{0}$-semigrupo $T$ em $X$, então $D(A)$ é denso em $X$.

Demonstração.

Seja $x \in X$. Para todo $t>0$,

$$
\frac{1}{t} \int_{0}^{t} T(s) x d s \in D(A)
$$

$\mathrm{e}$

$$
\lim _{t \rightarrow 0} \frac{1}{t} \int_{0}^{t} T(s) x d s=x
$$

como desejado.

O próximo resultado fornece uma representação integral para o resolvente do gerador de um $C_{0}$-semigrupo.

Proposição 3.11. (Representação integral do resolvente)

Seja $T$ um $C_{0}$-semigrupo com gerador $A$ e $\lambda \in \mathbb{C}$. Se $\operatorname{Re}(\lambda)>\omega_{0}$, onde $\omega_{0}$ é o limite de crescimento de $T$, então $\lambda \in \rho(A) e$

$$
R(\lambda, A) x=\int_{0}^{\infty} e^{-\lambda t} T(t) x d t .
$$

Demonstração. 
Note que $S(t)=e^{-\lambda t} T(t)$ é um $C_{0}$-semigrupo com gerador $(A-\lambda)$. Pelo Lema 3.9, $\int_{0}^{t} S(s) x d s \in D(A) \mathrm{e}$

$$
(A-\lambda) \int_{0}^{t} S(s) x d s=S(t) x-x .
$$

Pela Proposição 3.4, existe $M>0$ tal que $\|S(t)\| \leq M e^{(\omega-\operatorname{Re} \lambda) t}$, para todo $\omega>\omega_{0}$. Logo $\int_{0}^{\infty} S(s) x d s$ existe e

$$
\lim _{t \rightarrow \infty}(A-\lambda) \int_{0}^{t} S(s) x d s=\lim _{t \rightarrow \infty} S(t) x-x=-x
$$

Como $(A-\lambda)$ é fechado, então $\int_{0}^{\infty} S(s) x d s \in D(A)$ e

$$
(A-\lambda) \int_{0}^{\infty} S(s) x d s=-x
$$

para todo $x \in X$.

Reciprocamente, pela Proposição 3.3, temos

$$
\lim _{t \rightarrow \infty} \int_{0}^{t} S(s)(A-\lambda) x d s=\lim _{t \rightarrow \infty}(A-\lambda) \int_{0}^{t} S(s) x d s=-x
$$

para todo $x \in D(A)$. Logo

$$
\int_{0}^{\infty} e^{-\lambda t} T(t) x d t=(\lambda-A)^{-1} x
$$

como desejado.

Os próximos dois lemas são utilizados para provar a Proposição 3.14, que, por sua vez, será utilizada posteriormente para mostrar que o operador Laplaciano em domínios limitados possui resolvente compacto.

\section{Lema 3.12.}

Seja $X$ um espaço de Banach, $K \subset \mathbb{R}$ um compacto e considere a função $T: K \rightarrow$ $\mathcal{L}(X)$. Se, para todo $x \in X$, a função

$$
T_{x}: K \ni t \mapsto T(t) x \in X
$$


é contínua, então, para todo conjunto compacto $C \subset X$, a função

$$
K \times C \ni(t, x) \mapsto T(t) x \in X
$$

é uniformemente contínua.

Demonstração. Para todo $x \in X$, a função $T_{x}$ é limitada e uniformemente contínua, já que pela hipótese ela é contínua e seu domínio é compacto. Como $T_{x}$ é limitada para todo $x \in X$, utilizamos o Teorema de Banach-Steinhaus 2.8 e obtemos

$$
\sup _{t \in K}\|T(t)\|=M<\infty
$$

Seja $C \subset X$ compacto e $\epsilon>0$. Como $C$ é compacto, então existe uma quantidade finita de pontos $x_{1}, x_{2}, \ldots, x_{m} \in X$ tal que $X \subset \cup_{j=1}^{m} B\left(x_{i}, \epsilon / M\right)$. Utilizando o fato que $T_{x}$ é uniformemente contínua para todo $x \in X$, temos que existe $\delta>0$ tal que se $|t-s|<\delta$, então $\left\|T(t) x_{j}-T(s) x_{j}\right\| \leq \epsilon$, para todo $t, s \in K$ e $j \in\{1,2, \ldots, m\}$. Portanto, para todo $(t, x),(s, y) \in K \times C$ com $|t-s|<\delta$ e $\|x-y\|<\epsilon / M$, escolhemos $j$ tal que $\left\|x-x_{j}\right\| \leq \epsilon / M$, e temos

$$
\begin{aligned}
\|T(t) x-T(s) y\| & =\left\|T(t) x-T(t) x_{j}+T(t) x_{j}-T(s) x_{j}+T(s) x_{j}-T(s) x+T(s) x-T(s) y\right\| \\
& \leq\left\|T(t)\left(x-x_{j}\right)\right\|+\left\|(T(t)-T(s)) x_{j}\right\|+\left\|T(s)\left(x_{j}-x\right)\right\|+\|T(s)(x-y)\| \\
& \leq 4 \epsilon,
\end{aligned}
$$

obtendo o desejado.

\section{Lema 3.13.}

Seja $T$ um $C_{0}$-semigrupo e $t_{0}>0$. Se $T\left(t_{0}\right)$ é compacto, então $T(t)$ é compacto, para todo $t \geq t_{0}$, e a função $\left[t_{0}, \infty\right) \ni t \mapsto T(t)$ é contínua na topologia da norma.

\section{Demonstração.}

Pela definição $C_{0}$-semigrupo e pela propriedade de ideal dos operadores compactos 2.27, $T\left(t_{0}+h\right)=T\left(t_{0}\right) T(h)$ é compacto, para todo $h \geq 0$.

Seja $B$ a bola unitária em $X$. Pela hipótese, $T\left(t_{0}\right)$ é compacto, logo o conjunto $C=\overline{T\left(t_{0}\right) B}$ é compacto. Utilizando o Lema 3.12, quando $s \rightarrow t$, temos

$$
T(t) x-T(s) x=T\left(t-t_{0}\right) T\left(t_{0}\right) x-T\left(s-t_{0}\right) T\left(t_{0}\right) x \rightarrow 0,
$$


uniformemente em $U$, para todo $t \geq t_{0}$.

\section{Proposição 3.14 .}

Seja $T$ um $C_{0}$-semigrupo com gerador $A$. Se $T(t)$ é compacto para todo $t>0$, então $(0, \infty) \ni t \mapsto T(t) \in \mathcal{L}(X)$ é contínuo e A tem resolvente compacto.

\section{Demonstração.}

A função $(0, \infty) \ni t \mapsto T(t) \in \mathcal{L}(X)$ é contínua como consequência do Lema 3.13.

Pela Proposição 3.11, tomando $\lambda>\omega_{0}$ temos $\lambda \in \rho(A)$. Note que $S(t)=e^{-\lambda t} T(t)$ é um $C_{0}$-semigrupo com gerador $(A-\lambda)$ e como $T(t)$ é compacto para todo $t>0$ então $S(t)$ é compacto para todo $t>0$. Pelo Lema 3.9, $\int_{0}^{t} S(s) x d s=R(\lambda, A) x-R(\lambda, A) S(t) x$. Logo, basta mostrar que $x \mapsto \int_{0}^{t} S(s) x d s$ é compacta. Note que $(0, t] \ni s \mapsto S(s) \in \mathcal{L}(X)$ é limitada e contínua, logo é integrável. De fato, $\int_{0}^{t} S(s) d s$ é o limite na norma do operador de somas de Riemann compactas, $\operatorname{logo} \int_{0}^{t} S(s) d s$ é compacto.

\subsection{Semigrupos autoadjuntos}

Esta seção mostra as propriedades dos semigrupos gerados por operadores autoadjuntos agindo num espaço de Hilbert $H$.

\section{Teorema 3.15 .}

Seja $A$ um operador em $H$ simétrico, m-dissipativo e com resolvente compacto. Então $A$ é gerador de um $C_{0}$-semigrupo $T$ e, para todo $t \in(0, \infty), T(t)$ é um operador compacto, autoadjunto e contrativo. Além do mais, se A for injetor, então existe $\epsilon>0$ tal que $\|T(t)\| \leq e^{-\epsilon t}$, para todo $t \in \mathbb{R}_{+}$.

\section{Demonstração.}

Pelo Corolário 2.51, a menos de uma equivalência unitária, podemos assumir $H=\ell^{2}$ e $A=M_{-\lambda}$, onde $\lambda=\left(\lambda_{n}\right)_{n \in \mathbb{N}}$ é uma sequência em $(0, \infty)$ e $\lambda_{n} \rightarrow \infty$. Seja $T(t) \in \mathcal{L}\left(\ell^{2}\right)$ definido por

$$
T(t) x=\left(e^{-\lambda_{n} t} x_{n}\right)_{n \in \mathbb{N}}
$$

Uma checagem direta da definição mostra que $T$ é um $C_{0}$-semigrupo e que $A=M_{-\lambda}$ é seu gerador. Pela Proposição 2.48 segue que $T(t)$ é compacto, autoadjunto e

$$
\|T(t)\| \leq 1
$$


Se $A$ é injetor, então existe $0<\epsilon<\lambda_{n}$, para todo $n \in \mathbb{N}$, e, portanto, $\|T(t)\| \leq e^{-\epsilon t}$, para todo $t \in(0, \infty)$.

A seguir, temos a versão do mesmo teorema para operadores sem resolvente compacto.

\section{Teorema 3.16.}

Seja A um operador em $H$ autoadjunto e dissipativo. Então A é gerador de um $C_{0}$-semigrupo $T$ e, para todo $t \in \mathbb{R}_{+}, T(t)$ é um operador autoadjunto e contrativo.

Demonstração.

Pelo Teorema 2.52, a menos de uma equivalência unitária, podemos assumir $H=$ $L^{2}(Y)$ e $A=M_{-a}$, onde $Y$ é um espaço de medida finito e $a: Y \rightarrow \mathbb{R}_{+}$é uma função mensurável. Seja $T(t) \in \mathcal{L}\left(L^{2}(Y)\right)$ definido por $T(t) x=e^{-a t} x$. Uma checagem direta da definição mostra que $T$ é um $C_{0}$-semigrupo e que $A=M_{-a}$ é seu gerador. Pela Proposição 2.46 segue que $T(t)$ é autoadjunto e $\|T(t)\| \leq\left\|e^{-a t}\right\|_{\infty} \leq 1$. 


\section{Transformada de Fourier}

Neste capítulo, apresentaremos os fatos básicos sobre a transformada de Fourier. Na primeira seção, estudamos a transformada de Fourier no espaço das funções integráveis e mostramos suas principais propriedades. A segunda seção introduz o espaço de Schwartz, o que permite estender a transformada de Fourier para um operador unitário em $L^{2}\left(\mathbb{R}^{n}\right)$. Por fim, na terceira seção, analisamos a transformada de Fourier no espaço das distribuições temperadas. O ferramental desenvolvido permite a dedução do núcleo do calor, que por sua vez terá grande importância na demonstração da Lei de Weyl.

Os principais textos consultados para a elaboração deste capítulo foram [1], [53] e [20].

\subsection{Propriedades básicas}

Neste capítulo utilizaremos a notação multi-índice, que tem a seguinte definição:

Definição 4.1. (Notação multi-índice)

Seja $\mathbb{N}_{0}$ o conjunto dos números naturais incluindo o número zero e $n \in \mathbb{N}$. Chamamos $\alpha$ de um multi-índice de dimensão $n$ se $\alpha=\left(\alpha_{1}, \alpha_{2}, \ldots, \alpha_{n}\right) \in \mathbb{N}_{0}^{n}$. Sejam $\alpha=$ $\left(\alpha_{1}, \alpha_{2}, \ldots, \alpha_{n}\right), \beta=\left(\beta_{1}, \beta_{2}, \ldots, \beta_{n}\right) \in \mathbb{N}_{0}^{n}$ e $x=\left(x_{1}, x_{2}, \ldots, x_{n}\right) \in \mathbb{R}^{n}$. São feitas as seguintes definições:

(a) $\alpha \leq \beta \Longleftrightarrow \alpha_{j} \leq \beta_{j}, \forall j \in 1, \ldots, n$;

(b) $\alpha+\beta=\left(\alpha_{1}+\beta_{1}, \alpha_{2}+\beta_{2}, \ldots, \alpha_{n}+\beta_{n}\right)$;

(c) se $\beta \leq \alpha \Longrightarrow \alpha-\beta=\left(\alpha_{1}-\beta_{1}, \alpha_{2}-\beta_{2}, \ldots, \alpha_{n}-\beta_{n}\right)$;

(d) $|\alpha|=\alpha_{1}+\alpha_{2}+\ldots+\alpha_{n}$;

(e) $\alpha !=\alpha_{1} ! \alpha_{2} ! \ldots \alpha_{n} !$

(f) $x^{\alpha}=x_{1}^{\alpha_{1}} x_{2}^{\alpha_{2}} \ldots x_{n}^{\alpha_{n}}$

(g) $\partial^{\alpha}=\partial_{1}^{\alpha_{1}} \partial_{2}^{\alpha_{2}} \ldots \partial_{n}^{\alpha_{n}}$, onde $\partial_{j}^{\alpha_{j}}=\partial^{\alpha_{j}} / \partial x_{j}^{\partial_{j}}$;

(h) se $\beta \leq \alpha \Longrightarrow\left(\begin{array}{l}\alpha \\ \beta\end{array}\right)=\left(\begin{array}{c}\alpha_{1} \\ \beta_{1}\end{array}\right)\left(\begin{array}{c}\alpha_{2} \\ \beta_{2}\end{array}\right) \cdots\left(\begin{array}{c}\alpha_{n} \\ \beta_{n}\end{array}\right)=\frac{\alpha !}{\beta !(\alpha-\beta) !}$.

Começamos com a definição de convolução que, veremos mais adiante, se relaciona de maneira interessante com a transformada de Fourier. 
Definição 4.2. (Convolução)

Sejam $f, g: \mathbb{R}^{n} \rightarrow \mathbb{C}$ funções mensuráveis. A convolução de $f$ e $g$ é a função

$$
(f * g)(x):=\int_{\mathbb{R}^{n}} f(x-y) g(y) d y
$$

definida para todo $x \in \mathbb{R}^{n}$ para o qual a integral existe.

A convolução está bem definida para quase-todo ponto quando $f \in L^{1}$ e $g \in L^{p}$. Isto faz parte da Desigualdade de Young enunciada abaixo.

Proposição 4.3. (Desigualdade de Young)

Se $f \in L^{1}\left(\mathbb{R}^{n}\right)$ e $g \in L^{p}\left(\mathbb{R}^{n}\right)$ comp $\in[1, \infty]$, então $f * g \in L^{p}\left(\mathbb{R}^{n}\right)$ e $\|f * g\|_{p} \leq\|f\|_{1}\|g\|_{p}$.

Inicialmente definimos a transformada de Fourier para funções integráveis. Posteriormente ampliaremos este domínio.

Definição 4.4. (Transformada de Fourier em $L^{1}\left(\mathbb{R}^{n}\right)$ )

Seja $f \in L^{1}\left(\mathbb{R}^{n}\right)$. A transformada de Fourier de $f$, denotada por $\mathcal{F}[f]$ ou $\hat{f}$, é a função $\mathcal{F}[f]: \mathbb{R}^{n} \rightarrow \mathbb{C}$ definida por:

$$
\mathcal{F}[f](\xi)=\hat{f}(\xi):=\int_{\mathbb{R}^{n}} e^{-2 \pi i x \cdot \xi} f(x) d x
$$

Como $\left|e^{-2 \pi i x \cdot \xi} f(x)\right|=|f(x)|$, para todo $\xi \in \mathbb{R}^{n}$, então $x \mapsto e^{-2 \pi i x \cdot \xi} f(x) \in L^{1}\left(\mathbb{R}^{n}\right)$ e a transformada de Fourier de $f$ está bem definida. Além do mais, segue da linearidade da integral que $\mathcal{F}$ é linear. A seguir seguem algumas propriedades importantes.

Proposição 4.5. (Translação, modulação, dilatação)

Seja $f \in L^{1}\left(\mathbb{R}^{n}\right), x, y \in \mathbb{R}^{n}$ e $a>0$, então:

(a) $\mathcal{F}[f(x+y)](\xi)=e^{2 \pi i y \cdot \xi} \hat{f}(\xi)$.

(b) $\mathcal{F}\left[e^{2 \pi i x \cdot y} f(x)\right](\xi)=\hat{f}(\xi-y)$.

(c) $\mathcal{F}[f(a x)](\xi)=a^{-n} \hat{f}(\xi / a)$.

Demonstração.

Está claro que $x \mapsto f(x+y), x \mapsto e^{2 \pi i x \cdot y} f(x), x \mapsto f(a x)$ são integráveis. 
(a) Fazendo a mudança de variável $x \rightarrow x-y$, temos

$$
\begin{aligned}
\mathcal{F}[f(x+y)](\xi) & =\int_{\mathbb{R}^{n}} e^{-2 \pi i x \cdot \xi} f(x+y) d x \\
& =\int_{\mathbb{R}^{n}} e^{-2 \pi i(x-y) \cdot \xi} f(x) d x \\
& =e^{2 \pi i y \cdot \xi} \int_{\mathbb{R}^{n}} e^{-2 \pi i x \cdot \xi} f(x) d x \\
& =e^{2 \pi i y \cdot \xi} \hat{f}(\xi) .
\end{aligned}
$$

(b)

$$
\begin{aligned}
\mathcal{F}\left[e^{-2 \pi i y \cdot x} f(x)\right](\xi) & =\int_{\mathbb{R}^{n}} e^{2 \pi i x \cdot y} e^{-2 \pi i x \cdot \xi} f(x) d x \\
& =\int_{\mathbb{R}^{n}} e^{-2 \pi i x \cdot(\xi-y)} f(x) d x \\
& =\hat{f}(\xi-y) .
\end{aligned}
$$

(c) Fazendo mudança de variável $x \rightarrow x / a$, temos

$$
\begin{aligned}
\mathcal{F}[f(a x)](\xi) & =\int_{\mathbb{R}^{n}} e^{-2 \pi i x \cdot \xi} f(a x) d x \\
& =\int_{\mathbb{R}^{n}} e^{-2 \pi i(x / a) \cdot \xi} f(x) a^{-n} d x \\
& =a^{-n} \hat{f}(\xi / a),
\end{aligned}
$$

como desejado.

A transformada de Fourier transforma uma convolução em uma multiplicação de funções.

Proposição 4.6. (Transformada de Fourier e convolução)

Sejam $f, g \in L^{1}\left(\mathbb{R}^{n}\right)$, então $\mathcal{F}(f * g)=\hat{f} \hat{g}$.

Demonstração.

Pela Desigualdade de Young 4.3, $f * g \in L^{1}\left(\mathbb{R}^{n}\right)$. Aplicando o Teorema de Fubini 
temos

$$
\begin{aligned}
\mathcal{F}(f * g)(\xi) & =\int_{\mathbb{R}^{n}} \int_{\mathbb{R}^{n}} f(x-y) g(y) e^{-2 \pi i x \cdot \xi} d y d x \\
& =\int_{\mathbb{R}^{n}} \int_{\mathbb{R}^{n}} f(x-y) e^{-2 \pi i(x-y) \cdot \xi} g(y) e^{-2 \pi i y \cdot \xi} d x d y \\
& =\hat{f}(\xi) \int_{\mathbb{R}^{n}} g(y) e^{-2 \pi i y \cdot \xi} d y \\
& =\hat{f}(\xi) \hat{g}(\xi)
\end{aligned}
$$

como desejado.

A transformada de Fourier de uma função integrável é contínua.

Proposição 4.7. (Continuidade)

A transformada de Fourier $\mathcal{F}: L^{1}\left(\mathbb{R}^{n}\right) \rightarrow L^{\infty}\left(\mathbb{R}^{n}\right)$ é contínua e sua imagem consiste de funções contínuas.

Demonstração.

Temos

$$
\begin{aligned}
\|\hat{f}\|_{L^{\infty}\left(\mathbb{R}^{n}\right)} & =\sup _{\xi \in \mathbb{R}^{n}}\left|\int_{\mathbb{R}^{n}} e^{-2 \pi i x \cdot \xi} f(x) d x\right| \\
& \leq \sup _{\xi \in \mathbb{R}^{n}} \int_{\mathbb{R}^{n}}\left|e^{-2 \pi i x \cdot \xi}\right||f(x)| d x \\
& =\|f\|_{L^{1}\left(\mathbb{R}^{n}\right)} .
\end{aligned}
$$

Logo $\mathcal{F}$ é contínua e $\hat{f} \in L^{\infty}\left(\mathbb{R}^{n}\right)$. Para ver a continuidade de $\hat{f}$, note que:

$$
\begin{aligned}
|\hat{f}(\xi+\eta)-\hat{f}(\xi)| & =\left|\int_{\mathbb{R}^{n}}\left(e^{-2 \pi i x \cdot(\xi+\eta)}-e^{-2 \pi i x \cdot \xi}\right) f(x) d x\right| \\
& =\left|\int_{\mathbb{R}^{n}}\left(e^{-2 \pi i x \cdot \eta}-1\right) e^{-2 \pi i x \cdot \xi} f(x) d x\right| \\
& \leq \int_{\mathbb{R}^{n}}\left|e^{-2 \pi i x \cdot \eta}-1\right||f(x)| d x
\end{aligned}
$$

O integrando $\left|e^{-2 \pi i x \cdot \eta}-1\right||f(x)|$ é limitado por $2|f(x)|$ e tende a zero quando $\eta \rightarrow 0$. Logo o resultado é consequência do Teorema da Convergência Dominada.

O próximo resultado mostra que a diferenciabilidade de $f$ implica no decaimento polinomial de $\hat{f}$ e vice-versa. Para demonstrá-lo, utilizamos o seguinte lema. 


\section{Lema 4.8.}

Seja I um intervalo aberto em $\mathbb{R}$ e $f: \mathbb{R}^{n} \times I \rightarrow \mathbb{C}$ tal que $f(x, \cdot)$ é diferenciável para todo $x \in \mathbb{R}^{n}, f(\cdot, t)$ é integrável em $\mathbb{R}^{n}$ para todo $t \in I$ e existe $g \in L^{1}\left(\mathbb{R}^{n}\right)$ tal que $\left|\partial_{t} f(x, t)\right| \leq g(x)$ para quase todo $x \in \mathbb{R}^{n}$. Então $F(t):=\int_{\mathbb{R}^{n}} f(x, t) d x$ é diferenciável e $F^{\prime}(t)=\int_{\mathbb{R}^{n}} \partial_{t} f(x, t) d x$.

Proposição 4.9. (Derivada da transformada de Fourier)

(a) Se $x^{\alpha} f \in L^{1}\left(\mathbb{R}^{n}\right)$ para todo $|\alpha| \leq k$, então $\hat{f} \in C^{k}\left(\mathbb{R}^{n}\right)$ e

$$
\left(\partial^{\alpha} \hat{f}\right)(\xi)=\mathcal{F}\left[(-2 \pi i x)^{\alpha} f(x)\right](\xi) .
$$

(b) Se $f \in C^{k}\left(\mathbb{R}^{n}\right)$ e $\partial^{\alpha} f \in L^{1}\left(\mathbb{R}^{n}\right)$ para todo $|\alpha| \leq k$, então

$$
\mathcal{F}\left[\partial^{\alpha} f\right](\xi)=(2 \pi i \xi)^{\alpha} \hat{f}(\xi)
$$

Demonstração.

(a) Primeiro assuma $|\alpha|=1$. Neste caso:

$$
\left(\partial^{\alpha} \hat{f}\right)(\xi)=\left(\partial_{\xi_{j}} \hat{f}\right)(\xi)=\partial_{\xi_{j}} \int_{\mathbb{R}^{n}} e^{-2 \pi i x \xi} f(x) d x
$$

Obtém-se o resultado utilizando o Lema 4.8, já que $\left|\partial_{\xi_{j}} e^{-2 \pi i x \xi} f(x)\right| \leq\left|2 \pi x_{j}\right||f(x)| \in$ $L^{1}\left(\mathbb{R}^{n}\right)$. O caso geral segue por indução em $|\alpha|$.

(b) Primeiro assuma $n=|\alpha|=1$. Se $f \in C^{1}(\mathbb{R}), f$ e $f^{\prime} \in L^{1}(\mathbb{R})$, então

$$
\lim _{x \rightarrow \infty} f(x)=f(0)+\int_{0}^{\infty} f^{\prime}(t) d t \in \mathbb{R}
$$

Logo $\lim _{x \rightarrow \infty} f(x)$ existe e, como $f$ é integrável, então $\lim _{x \rightarrow \infty} f(x)=0$. Podemos então utilizar a integração por partes e temos

$$
\begin{aligned}
\int_{-\infty}^{\infty} e^{-2 \pi i x \xi} f^{\prime}(x) d x & =\left.e^{-2 \pi i x \xi} f(x)\right|_{-\infty} ^{\infty}-\int_{-\infty}^{\infty}(-2 \pi i \xi) e^{-2 \pi i x \xi} f(x) d x \\
& =2 \pi i \xi \int_{-\infty}^{\infty} e^{-2 \pi i x \xi} f(x) d x
\end{aligned}
$$


No caso em que $n>1$ e $|\alpha|=1$, o mesmo argumento é utilizado, desta vez integrando por partes na j-ésima variável. O caso geral segue por indução em $|\alpha|$.

\subsection{Espaço de Schwartz}

O espaço de Schwartz é o espaço ideal para o estudo da transformada de Fourier, já que ela é um isomorfismo nesse espaço.

Definição 4.10. (Espaço de Schwartz)

O espaço de Schwartz, também chamado de espaço das funções suaves com decrescimento rápido, é definido por

$$
\mathcal{S}\left(\mathbb{R}^{n}\right):=\left\{\varphi \in C^{\infty}\left(\mathbb{R}^{n}\right): \forall \alpha, \beta \in \mathbb{N}_{0}^{n},\|\varphi\|_{\alpha, \beta}<\infty\right\}
$$

onde

$$
\|\varphi\|_{\alpha, \beta}:=\sup _{x \in \mathbb{R}^{n}}\left|x^{\alpha} D^{\beta} \varphi(x)\right|
$$

define uma família enumerável de semi-normas que torna $\mathcal{S}\left(\mathbb{R}^{n}\right)$ um espaço de Fréchet. Em outras palavras, uma função infinitamente diferenciável é de Schwartz se ela e todas as suas derivadas decrescem para zero no infinito mais rápido que o crescimento de qualquer polinômio. Uma sequência de funções $\left(\varphi_{j}\right)_{j \in \mathbb{N}}$ em $\mathcal{S}\left(\mathbb{R}^{n}\right)$ converge para $0 \in \mathcal{S}\left(\mathbb{R}^{n}\right)$ se, para todo $\alpha, \beta \in \mathbb{N}_{0}^{n}$, temos

$$
\lim _{j \rightarrow \infty}\left\|\varphi_{j}\right\|_{\alpha, \beta}=0
$$

Primeiro mostramos que a transformada de Fourier é contínua no espaço de Schwartz.

Proposição 4.11. (Transformada de Fourier em $\mathcal{S}\left(\mathbb{R}^{n}\right)$ )

Se $\varphi \in \mathcal{S}\left(\mathbb{R}^{n}\right)$, então $\hat{\varphi} \in \mathcal{S}\left(\mathbb{R}^{n}\right)$ e $\mathcal{F}: \mathcal{S}\left(\mathbb{R}^{n}\right) \rightarrow \mathcal{S}\left(\mathbb{R}^{n}\right)$ é contínua.

Demonstração.

Seja $\varphi \in \mathcal{S}\left(\mathbb{R}^{n}\right)$ e $\alpha, \beta \in \mathbb{N}_{0}^{n}$. Pela Proposição 4.9 , temos

$$
\left|\xi^{\alpha}\left(\partial^{\beta} \hat{\varphi}\right)(\xi)\right|=\left|\xi^{\alpha} \mathcal{F}\left[(-2 \pi i x)^{\beta} \varphi(x)\right](\xi)\right|=\left|\mathcal{F}\left[\partial^{\alpha}\left((-2 \pi i x)^{\beta} \varphi(x)\right)\right](\xi)\right|
$$

Como $g(x)=\partial^{\alpha}\left((-2 \pi i x)^{\beta} \varphi(x)\right) \in \mathcal{S}\left(\mathbb{R}^{n}\right) \subset L^{1}\left(\mathbb{R}^{n}\right)$, então

$$
\sup _{\xi \in \mathbb{R}^{n}}\left|\xi^{\alpha}\left(\partial^{\beta} \hat{\varphi}\right)(\xi)\right|=\sup _{\xi \in \mathbb{R}^{n}}|\hat{g}(\xi)| \leq\|g\|_{1}<\infty
$$


Logo $\hat{\varphi} \in \mathcal{S}\left(\mathbb{R}^{n}\right)$. Para provar a continuidade de $\mathcal{F}$, considere $\left(\varphi_{j}\right)_{j \in \mathbb{N}}$ uma sequência em $\mathcal{S}\left(\mathbb{R}^{n}\right)$, tal que $\varphi_{j} \rightarrow \varphi \in \mathcal{S}\left(\mathbb{R}^{n}\right)$, $\log$ $\varphi_{j} \rightarrow \varphi$ em $L^{1}\left(\mathbb{R}^{n}\right)$. Pela Proposição 4.7, $\hat{\varphi}_{j}(x) \rightarrow \hat{\varphi}(x)$, para todo $x \in \mathbb{R}^{n}$. O resultado segue do Teorema do Gráfico Fechado 2.9 .

Os próximos dois resultados serão utilizados para obter a transformada de Fourier inversa.

\section{Proposição 4.12 .}

Seja $a>0$ e $f(x)=e^{-\pi a|x|^{2}}$, então $\hat{f}(\xi)=a^{-n / 2} e^{-\pi|\xi|^{2} / a}$.

\section{Demonstração.}

Basta considerar o caso unidimensional já que, pelo Teorema de Fubini, $\hat{f}(\xi)=$ $\prod_{j=1}^{n} \int_{\mathbb{R}^{n}} e^{-2 \pi i \xi_{j} x_{j}-\pi a x_{j}^{2}} d x_{j}$. Utilizando a Proposição 4.9, temos

$$
\hat{f}^{\prime}(\xi)=\mathcal{F}\left[-2 \pi i x e^{-\pi a x^{2}}\right](\xi)=i a^{-1} \mathcal{F}\left[f^{\prime}\right](\xi)=-2 \pi a^{-1} \xi \hat{f}(\xi) .
$$

Além do mais, $\hat{f}(0)=\int_{\mathbb{R}} e^{-\pi a x^{2}} d x=a^{-1 / 2}$. Logo $\hat{f}$ fica unicamente determinada por $\hat{f}(\xi)=a^{-1 / 2} e^{-\pi \xi^{2} / a}$.

\section{Lema 4.13.}

Se $f, g \in L^{1}\left(\mathbb{R}^{n}\right)$, então

$$
\int_{\mathbb{R}^{n}} \hat{f}(x) g(x) d x=\int_{\mathbb{R}^{n}} f(x) \hat{g}(x) d x .
$$

Demonstração. A integral está bem definida, já que pela Proposição 4.7, $\hat{f} \in L^{\infty}\left(\mathbb{R}^{n}\right)$. Aplicando o Teorema de Fubini, temos

$$
\int_{\mathbb{R}^{n}} \hat{f}(x) g(x) d x=\int_{\mathbb{R}^{n}} \int_{\mathbb{R}^{n}} e^{-2 \pi i y \cdot x} f(y) g(x) d y d x=\int_{\mathbb{R}^{n}} f(x) \hat{g}(x) d x
$$

como esperado.

No espaço de Schwartz, podemos recuperar a função $f$ de sua transformada de Fourier $\hat{f}$.

Proposição 4.14. (Transformada de Fourier inversa) 
A transformação de Fourier $\mathcal{F}: \mathcal{S}\left(\mathbb{R}^{n}\right) \rightarrow \mathcal{S}\left(\mathbb{R}^{n}\right)$ possui inversa contínua definida por:

$$
\mathcal{F}^{-1}[f](x)=\check{f}(x):=\int_{\mathbb{R}^{n}} e^{2 \pi i x \cdot \xi} f(\xi) d \xi
$$

A função $\mathcal{F}^{-1}[f]: \mathbb{R}^{n} \rightarrow \mathbb{C}$ é chamada de transformada de Fourier inversa de $f$. Note que $\mathcal{F}^{-1}[f](x)=\mathcal{F}[f](-x)$.

Demonstração. Seja $\epsilon>0, \phi(\xi)=e^{-\pi|\xi|^{2}}$ e $g(\xi)=e^{2 \pi i x \cdot \xi-\pi \epsilon^{2}|\xi|^{2}}$. Pelas Proposições 4.5 e 4.12, a transformada de Fourier de $g$ é dada por:

$$
\hat{g}(\eta)=\epsilon^{-n} e^{-\pi|x-\eta|^{2} / \epsilon^{2}}=\epsilon^{-n} \phi((x-\eta) / \epsilon):=\phi_{\epsilon}(x-\eta) .
$$

Pelo Lema 4.13, temos

$$
\begin{aligned}
\int_{\mathbb{R}^{n}} e^{2 \pi i x \cdot \xi-\pi \epsilon^{2}|\xi|^{2}} \hat{f}(\xi) d \xi & =\int_{\mathbb{R}^{n}} g(\xi) \hat{f}(\xi) d \xi \\
& =\int_{\mathbb{R}^{n}} f(\eta) \hat{g}(\eta) d \eta \\
& =\int_{\mathbb{R}^{n}} f(\eta) \phi_{\epsilon}(x-\eta) d \eta \\
& =\left(f * \phi_{\epsilon}\right)(x) .
\end{aligned}
$$

Por um lado, utilizando o Teorema da Convergência Dominada e o fato de que $\hat{f} \in$ $L^{1}\left(\mathbb{R}^{n}\right)$, temos

$$
\lim _{\epsilon \rightarrow 0} \int_{\mathbb{R}^{n}} e^{2 \pi i x \cdot \xi-\pi \epsilon^{2}|\xi|^{2}} \hat{f}(\xi) d \xi=\int_{\mathbb{R}^{n}} e^{2 \pi i x \cdot \xi} \hat{f}(\xi) d \xi=\check{\hat{f}}(x)
$$

Por outro lado $f * \phi_{\epsilon} \rightarrow\left(\int_{\mathbf{R}^{n}} e^{-\pi|\xi|^{2}} d \xi\right) f=f$ converge uniformemente também pelo Teorema da Convergência Dominada, quando $\epsilon \rightarrow 0$. Logo $\tilde{\hat{f}}=f$ e analogamente $\hat{\tilde{f}}=$ $f$.

\section{Corolário 4.15.}

A transformada de Fourier $\mathcal{F}$ é um isomorfismo em $\mathcal{S}\left(\mathbb{R}^{n}\right)$.

\section{Proposição 4.16.}

Se $f, g \in \mathcal{S}\left(\mathbb{R}^{n}\right)$ e $\alpha \in \mathbb{N}_{0}^{n}$, então $f * g=g * f \in \mathcal{S}\left(\mathbb{R}^{n}\right)$ e $\partial^{\alpha}(f * g)=\left(\partial^{\alpha} f\right) * g=f *\left(\partial^{\alpha} g\right)$.

Demonstração. Pelas Proposições 4.6 e 4.11 temos $\mathcal{F}[f * g]=\hat{f} \hat{g} \in \mathcal{S}\left(\mathbb{R}^{n}\right)$. Logo, pelo 
Corolário 4.15, $f * g=\mathcal{F}^{-1}[\hat{f} \hat{g}]=g * f \in \mathcal{S}\left(\mathbb{R}^{n}\right)$. Utilizando 4.9, temos

$$
\begin{aligned}
\partial^{\alpha}(f * g)(x) & =\partial^{\alpha} \mathcal{F}^{-1}[\hat{f} \hat{g}](x) \\
& =\mathcal{F}^{-1}\left[(2 \pi i \xi)^{\alpha} \hat{f} \hat{g}\right](x) \\
& =\mathcal{F}^{-1}\left[\widehat{\partial^{\alpha} f} \hat{g}\right](x) \\
& =\left(\left(\partial^{\alpha} f\right) * g\right)(x) .
\end{aligned}
$$

Analogamente obtemos $\partial^{\alpha}(f * g)=f *\left(\partial^{\alpha} g\right)$.

O próximo resultado nos revela que podemos estender a transformada de Fourier para o espaço $L^{2}\left(\mathbb{R}^{n}\right)$.

\section{Proposição 4.17. (Teorema de Plancherel)}

A transformada de Fourier $\mathcal{F}: \mathcal{S}\left(\mathbb{R}^{n}\right) \rightarrow \mathcal{S}\left(\mathbb{R}^{n}\right)$ se estende de forma única a um operador unitário em $L^{2}\left(\mathbb{R}^{n}\right)$.

Demonstração. Primeiro vamos mostrar que $\|\varphi\|_{2}=\|\hat{\varphi}\|_{2}$, para todo $\varphi \in \mathcal{S}\left(\mathbb{R}^{n}\right)$. Utilizando o Teorema de Fubini e o Teorema 4.14, temos

$$
\begin{aligned}
\|\hat{\varphi}\|_{2}^{2} & =\int_{\mathbb{R}^{n}} \hat{\varphi}(\xi) \overline{\hat{\varphi}}(\xi) d \xi \\
& =\int_{\mathbb{R}^{n}} \int_{\mathbb{R}^{n}} e^{-2 \pi i \xi \cdot x} \varphi(x) d x \overline{\hat{\varphi}}(\xi) d \xi \\
& =\int_{\mathbb{R}^{n}} \varphi(x) \int_{\mathbb{R}^{n}} e^{-2 \pi i \xi \cdot x} \overline{\hat{\varphi}}(\xi) d \xi d x \\
& =\int_{\mathbb{R}^{n}} \varphi(x) \int_{\mathbb{R}^{n}} e^{2 \pi i \xi \cdot x} \hat{\varphi}(\xi) d \xi d x \\
& =\int_{\mathbb{R}^{n}} \varphi(x) \bar{\varphi}(x) d x . \\
& =\|\varphi\|_{2}^{2} .
\end{aligned}
$$

Como $\mathcal{S}\left(\mathbb{R}^{n}\right)$ é denso em $L^{2}\left(\mathbb{R}^{n}\right)$, então $\mathcal{F}$ se estende de forma única para um operador contínuo $\mathcal{F}: L^{2}\left(\mathbb{R}^{n}\right) \rightarrow L^{2}\left(\mathbb{R}^{n}\right)$. O mesmo vale para $\mathcal{F}^{-1}$. Para toda $f \in L^{2}\left(\mathbb{R}^{n}\right)$, $\mathcal{F}^{-1}(\hat{f})=f$, já que isso vale para toda $f \in \mathcal{S}\left(\mathbb{R}^{n}\right)$.

\subsection{Distribuições temperadas}

Distribuições generalizam a noção de função com a vantagem de serem infinitamente diferenciáveis. A ideia de função que assume valores em pontos é reinterpretada como um 
funcional linear que age sobre funções testes. Quando o espaço de Schwartz é utilizado como espaço de funções testes, obtemos as distribuições temperadas. O benefício desse uso é que a transformada de Fourier é um isomorfismo no espaço das distribuições temperadas. Além disso, funções de Schwartz e funções $L^{p}$ estão contidas (naturalmente) no espaço das distribuições temperadas.

Definição 4.18. (Distribuições temperadas)

O espaço das distribuições temperadas, denotado por $\mathcal{S}^{\prime}\left(\mathbb{R}^{n}\right)$, é o espaço dual contínuo do espaço de Schwartz, eu seja, é o espaço dos funcionais lineares $T: \mathcal{S}\left(\mathbb{R}^{n}\right) \rightarrow \mathbb{C}$ tais que, para toda sequência de funções $\left(\varphi_{j}\right)_{j \in \mathbb{N}}$ em $\mathcal{S}\left(\mathbb{R}^{n}\right)$ convergente para $0 \in \mathcal{S}\left(\mathbb{R}^{n}\right)$, temos $\lim _{j \rightarrow \infty} T\left(\varphi_{j}\right)=0$. Dizemos que uma sequência $\left(T_{j}\right)_{j \in \mathbb{N}}$ em $\mathcal{S}^{\prime}\left(\mathbb{R}^{n}\right)$ converge para $T \in \mathcal{S}^{\prime}\left(\mathbb{R}^{n}\right)$ se, e somente se, para toda função $\varphi \in \mathcal{S}\left(\mathbb{R}^{n}\right)$, temos

$$
\lim _{j \rightarrow \infty} T_{j}(\varphi)=T(\varphi)
$$

Funções temperadas são associadas de maneira natural a distribuições temperadas. $\mathrm{O}$ contrário em geral não é verdadeiro.

Definição 4.19. (Função temperada)

Uma função mensurável $f: \mathbb{R}^{n} \rightarrow \mathbb{C}$ é uma função temperada se existe $N \in \mathbb{N}$ tal que:

$$
\int_{\mathbb{R}^{n}} \frac{f(x)}{(1+|x|)^{N}} d x<\infty
$$

\section{Exemplo 4.20.}

Seja $p \in[1, \infty]$ e $f \in L^{p}\left(\mathbb{R}^{n}\right)$, então $f$ é uma função temperada. A prova segue da Desigualdade de Holder.

\section{Proposição 4.21.}

Seja $f$ uma função temperada, então o funcional linear definido por

$$
T_{f}(\varphi):=\int_{\mathbb{R}^{n}} f(x) \varphi(x) d x
$$

para todo $\varphi \in \mathcal{S}\left(\mathbb{R}^{n}\right)$, é uma distribuição temperada.

Demonstração. 
Primeiro mostramos que o funcional linear está bem definido. De fato,

$$
\begin{aligned}
\left|T_{f}(\varphi)\right| & \leq \int_{\mathbb{R}^{n}}|f(x)||\varphi(x)| d x \\
& =\int_{\mathbb{R}^{n}} \frac{|f(x)|}{(1+|x|)^{N}}(1+|x|)^{N}|\varphi(x)| d x \\
& \leq \int_{\mathbb{R}^{n}} \frac{|f(x)|}{(1+|x|)^{N}} d x \sup _{x \in \mathbb{R}^{n}}\left\{(1+|x|)^{N}|\varphi(x)|\right\}<\infty .
\end{aligned}
$$

Para mostrar que $T_{f}$ é contínuo, seja $\left(\varphi_{j}\right)_{j \in \mathbb{N}}$ uma sequência de funções em $\mathcal{S}\left(\mathbb{R}^{n}\right)$ convergente para $0 \in \mathcal{S}\left(\mathbb{R}^{n}\right)$. Temos

$$
\left|T_{f}\left(\varphi_{j}\right)\right| \leq \int_{\mathbb{R}^{n}} \frac{|f(x)|}{(1+|x|)^{N}} d x \sup _{x \in \mathbb{R}^{n}}\left\{(1+|x|)^{N}\left|\varphi_{j}(x)\right|\right\} \rightarrow 0 \text { quando } j \rightarrow \infty
$$

Logo $T_{f}$ é contínuo.

Definição 4.22. (Distribuição temperada regular)

Uma distribuição temperada $T$ é regular, se existe uma função temperada $f$ tal que $T(\varphi)=T_{f}(\varphi)$, para todo $\varphi \in \mathcal{S}\left(\mathbb{R}^{n}\right)$.

Exemplo 4.23. (Delta de Dirac)

Nem toda distribuição temperada é regular. Por exemplo, a distribuição delta de Dirac definida por $\delta(\varphi):=\varphi(0)$, para todo $\varphi \in \mathcal{S}\left(\mathbb{R}^{n}\right)$. De fato, suponha $\delta=T_{f}$. Se considerarmos apenas as funções teste que valem zero na origem, então

$$
\delta(\varphi)=\varphi(0)=0=\int_{\mathbb{R}^{n}} f(x) \varphi(x) d x=\int_{\mathbb{R}^{n} \backslash\{0\}} f(x) \varphi(x) d x .
$$

Logo, $\left.f\right|_{\mathbb{R}^{n} \backslash\{0\}}=0$ e $f$ é a função identicamente nula. Obtemos assim um absurdo.

Um recurso comum em certas demonstrações é o de aproximar uma distribuição por meio de uma sequência de funções suaves através da convolução, tal como feito na demonstração da Proposição 4.14. Nesse sentido é útil a seguinte definição.

Definição 4.24. (Molificador)

Um molificador é uma função $\varphi \in C_{c}^{\infty}\left(\mathbb{R}^{n}\right)$ tal que $\int_{\mathbb{R}^{n}} \varphi(x) d x=1$ e $\lim _{\epsilon \rightarrow 0} \varphi_{\epsilon}(x):=$ $\lim _{\epsilon \rightarrow 0} \epsilon^{-n} \varphi(x / \epsilon)=\delta(x)$, onde o limite deve ser entendido no espaço das distribuições temperadas. 


\section{Exemplo 4.25.}

Por exemplo, a função $\varphi \in C_{c}^{\infty}\left(\mathbb{R}^{n}\right)$ definida por

$$
\varphi(x)= \begin{cases}\frac{e^{-1 /\left(1-|x|^{2}\right)}}{\int_{-1}^{1} \varphi(x) d x} & \text { se }|x|<1 \\ 0 & \text { se }|x| \geq 1\end{cases}
$$

é um molificador.

Uma das principais características das distribuições temperadas é que elas são infinitamente diferenciáveis (em um sentido generalizado).

Definição 4.26. (Derivada distribucional)

Seja $T \in \mathcal{S}^{\prime}\left(\mathbb{R}^{n}\right)$ e $\alpha \in \mathbb{N}_{0}^{n}$. A derivada distribucional $\partial^{\alpha} T \in \mathcal{S}^{\prime}\left(\mathbb{R}^{n}\right)$ de $T$ é definida, para todo $\varphi \in \mathcal{S}\left(\mathbb{R}^{n}\right)$, por:

$$
\partial^{\alpha} T(\varphi):=(-1)^{|\alpha|} T\left(\partial^{\alpha} \varphi\right)
$$

\section{Observação 4.27.}

Note que se $f \in C^{k}\left(\mathbb{R}^{n}\right)$ e para todo $|\alpha| \leq k$ a função $\partial^{\alpha} f$ é temperada, então, utilizando a integração por partes, verificamos que a derivada distribucional coincide com a derivada usual. De fato,

$$
\begin{aligned}
\partial^{\alpha} T_{f}(\varphi) & =(-1)^{|\alpha|} \int_{\mathbb{R}^{n}} f(x) \partial^{\alpha} \varphi(x) d x \\
& =\int_{\mathbb{R}^{n}} \partial^{\alpha} f(x) \varphi(x) d x \\
& =T_{\partial^{\alpha} f}(\varphi) .
\end{aligned}
$$

Exemplo 4.28. (Função de Heaviside)

Considere a função de Heaviside $H: \mathbb{R} \rightarrow \mathbb{R}$ definida por $H(x)=1$ se $x \geq 0$ e $H(x)=0$ se $x<0$. A função de Heaviside é temperada e a derivada da distribuição temperada regular $T_{H}$ é a distribuição delta de Dirac. De fato

$$
\begin{aligned}
T_{H}^{\prime}(\varphi) & =-\int_{\mathbb{R}} H(x) \varphi^{\prime}(x) d x \\
& =-\int_{0}^{\infty} \varphi^{\prime}(x) d x \\
& =\varphi(0) \\
& =\delta(\varphi) .
\end{aligned}
$$


Definição 4.29. (Transformada de Fourier em $\mathcal{S}^{\prime}\left(\mathbb{R}^{n}\right)$ )

Seja $T \in \mathcal{S}^{\prime}\left(\mathbb{R}^{n}\right)$. Para todo $\varphi \in \mathcal{S}\left(\mathbb{R}^{n}\right)$, a transformada de Fourier $\hat{T}$ e a transformada de Fourier inversa $\check{T}$ de $T$ são definidas por:

$$
\begin{aligned}
& \hat{T}(\varphi):=T(\hat{\varphi}), \\
& \check{T}(\varphi):=T(\check{\varphi}) .
\end{aligned}
$$

\section{Observação 4.30.}

Note que esta definição generaliza a definição da transformada de Fourier em $\mathcal{S}\left(\mathbb{R}^{n}\right)$ e $L^{p}\left(\mathbb{R}^{n}\right)$, isto é, se $f \in L^{p}\left(\mathbb{R}^{n}\right)$, então $f$ é uma função temperada e a transformada de Fourier da distribuição associada $T_{f}$ é dada por:

$$
\begin{aligned}
\hat{T}_{f}(\varphi) & =\int_{\mathbb{R}^{n}} f(\xi) \hat{\varphi}(\xi) d \xi \\
& =\int_{\mathbb{R}^{n}} \int_{\mathbb{R}^{n}} e^{-2 \pi i \xi \cdot x} f(\xi) \varphi(x) d x d \xi \\
& =\int_{\mathbb{R}^{n}} \hat{f}(x) \varphi(x) d x \\
& =T_{\hat{f}}(\varphi) .
\end{aligned}
$$

O mesmo vale para a transformada de Fourier inversa.

\section{Proposição 4.31 .}

A transformada de Fourier $\mathcal{F}$ é um isomorfismo em $\mathcal{S}^{\prime}\left(\mathbb{R}^{n}\right)$.

Demonstração. Seja $T \in \mathcal{S}^{\prime}\left(\mathbb{R}^{n}\right)$. Para mostrar que $\hat{T} \in \mathcal{S}^{\prime}\left(\mathbb{R}^{n}\right)$ note que $\hat{T}(\varphi)=T(\hat{\varphi})=$ $(T \circ \mathcal{F})(\varphi)$, para todo $\varphi \in \mathcal{S}\left(\mathbb{R}^{n}\right)$. Como $\mathcal{F}: \mathcal{S}\left(\mathbb{R}^{n}\right) \rightarrow \mathcal{S}\left(\mathbb{R}^{n}\right)$ é um operador contínuo, $\log 0 \hat{T}=T \circ \mathcal{F} \in \mathcal{S}^{\prime}\left(\mathbb{R}^{n}\right)$.

Seja $\left(T_{j}\right)_{j \in \mathbb{N}}$ uma sequência em $\mathcal{S}^{\prime}\left(\mathbb{R}^{n}\right)$ tal que $T_{j} \rightarrow T \in \mathcal{S}^{\prime}\left(\mathbb{R}^{n}\right)$. Temos $\hat{T}_{j}(\varphi)=$ $T_{j}(\hat{\varphi}) \rightarrow T(\hat{\varphi})=\hat{T}(\varphi)$, quando $j \rightarrow \infty$. Logo $\mathcal{F}: \mathcal{S}^{\prime}\left(\mathbb{R}^{n}\right) \rightarrow \mathcal{S}^{\prime}\left(\mathbb{R}^{n}\right)$ é contínua. Analogamente $\mathcal{F}^{-1}: \mathcal{S}^{\prime}\left(\mathbb{R}^{n}\right) \rightarrow \mathcal{S}^{\prime}\left(\mathbb{R}^{n}\right)$ é contínua e $\check{\hat{T}}(\varphi)=\hat{\tilde{T}}(\varphi)=T(\check{\hat{\varphi}})=T(\varphi)$.

Exemplo 4.32. (Transformada de Fourier do delta de Dirac)

A transformada de Fourier da distribuição delta de Dirac é a distribuição associada a função temperada $f(x)=1$, de fato:

$$
\hat{\delta}(\varphi)=\delta(\hat{\varphi})=\hat{\varphi}(0)=\int_{\mathbb{R}^{n}} \varphi(x) d x=T_{1}(\varphi)
$$


Também podemos definir a convolução de uma distribuição temperada com uma função do espaço de Schwartz.

Definição 4.33. (Convolução em $\mathcal{S}^{\prime}\left(\mathbb{R}^{n}\right)$ )

Seja $T \in \mathcal{S}^{\prime}\left(\mathbb{R}^{n}\right)$ e $f \in \mathcal{S}\left(\mathbb{R}^{n}\right)$. A convolução $T * f \in \mathcal{S}^{\prime}\left(\mathbb{R}^{n}\right)$ é definida por:

$$
(T * f)(\varphi):=T(\tilde{f} * \varphi)
$$

para todo $\varphi \in \mathcal{S}\left(\mathbb{R}^{n}\right)$, onde $\tilde{f}(x):=f(-x)$.

\section{Observação 4.34 .}

Note que se $f, g \in \mathcal{S}\left(\mathbb{R}^{n}\right)$ e $T_{f}$ é a distribuição temperada regular associada a função temperada $f$, então $T_{f} * g=T_{f * g}$. De fato, para toda $\varphi \in \mathcal{S}\left(\mathbb{R}^{n}\right)$, temos

$$
\begin{aligned}
\left(T_{f} * g\right)(\varphi) & =T_{f}(\tilde{g} * \varphi) \\
& =\int_{\mathbb{R}^{n}} \int_{\mathbb{R}^{n}} f(x) g(y-x) \varphi(y) d y d x \\
& =T_{f * g}(\varphi) .
\end{aligned}
$$

\section{Exemplo 4.35.}

Seja $\delta$ a distribuição delta de Dirac e $f \in \mathcal{S}\left(\mathbb{R}^{n}\right)$, então $\delta * f=T_{f} \in \mathcal{S}^{\prime}\left(\mathbb{R}^{n}\right)$. Isto é, a distribuição delta de Dirac é o elemento neutro da convolução em $\mathcal{S}^{\prime}\left(\mathbb{R}^{n}\right)$. De fato, para toda $\varphi \in \mathcal{S}\left(\mathbb{R}^{n}\right)$, temos

$$
\begin{aligned}
(\delta * f)(\varphi) & =\delta(\tilde{f} * \varphi) \\
& =\int_{\mathbb{R}^{n}} f(y-0) \varphi(y) d y \\
& =T_{f}(\varphi)
\end{aligned}
$$

\section{Proposição 4.36.}

Seja $T \in \mathcal{S}^{\prime}\left(\mathbb{R}^{n}\right)$ e $f \in \mathcal{S}\left(\mathbb{R}^{n}\right)$, então $\partial^{\alpha}(T * f)=\left(\partial^{\alpha} T\right) * f=T *\left(\partial^{\alpha} f\right)$.

Demonstração. De fato, pela definição de convolução em $\mathcal{S}^{\prime}\left(\mathbb{R}^{n}\right)$ e pela Proposição 4.16 
temos

$$
\begin{aligned}
\partial^{\alpha}(T * f)(\varphi) & =(-1)^{|\alpha|}(T * f)\left(\partial^{\alpha} \varphi\right) \\
& =(-1)^{|\alpha|} T\left(\tilde{f} * \partial^{\alpha} \varphi\right) \\
& =(-1)^{|\alpha|} T\left(\partial^{\alpha}(\tilde{f} * \varphi)\right) \\
& =\left(\partial^{\alpha} T\right)(\tilde{f} * \varphi) \\
& =\left(\left(\partial^{\alpha} T\right) * f\right)(\varphi)
\end{aligned}
$$

e

$$
\begin{aligned}
\partial^{\alpha}(T * f)(\varphi) & =\left(\partial^{\alpha} T\right)(\tilde{f} * \varphi) \\
& =(-1)^{|\alpha|} T\left(\partial^{\alpha}(\tilde{f} * \varphi)\right) \\
& =(-1)^{|\alpha|} T\left(\left(\partial^{\alpha} \tilde{f}\right) * \varphi\right) \\
& =T\left(\widetilde{\left(\partial^{\alpha} f\right)} * \varphi\right) \\
& =\left(T *\left(\partial^{\alpha} f\right)\right)(\varphi),
\end{aligned}
$$

como desejado.

A transformada de Fourier no espaço das distribuições temperadas se mostra muito útil na resolução de equações diferenciais parciais, como nos exemplos abaixo.

Definição 4.37. (Solução fundamental)

Seja $L$ um operador linear diferencial com coeficientes constantes. Dizemos que $F \in$ $\mathcal{S}^{\prime}\left(\mathbb{R}^{n}\right)$ é a solução fundamental de $L$ se

$$
L F=\delta
$$

Se $u=F * f$ para alguma $f \in \mathcal{S}\left(\mathbb{R}^{n}\right)$, então $L u=f$. De fato

$$
L u=L(F * f)=L F * f=\delta * f=f .
$$

Note que $F$ não é única. Abaixo apresentaremos e deduziremos as soluções fundamentais mais comuns. 
Exemplo 4.38. (Equação de Laplace)

A solução fundamental $N$ para o Laplaciano é definida por:

$$
N(x)=\left\{\begin{array}{l}
\frac{\log |x|}{2 \pi}, \text { se } n=2, \\
\frac{|x|^{2-n}}{(2-n) \omega_{n}}, \text { se } n>2,
\end{array}\right.
$$

onde $\omega_{n}$ é a área da esfera unitária em $\mathbb{R}^{n}$. A função $N$ é temperada, logo define uma distribuição temperada regular $T_{N}$. Além do mais, podemos mostrar que

$$
\Delta T_{N}=\sum_{j=1}^{n} \partial_{j}^{2} T_{N}=\delta
$$

Seja $f \in \mathcal{S}\left(\mathbb{R}^{n}\right)$ e defina $u:=T_{N} * f \in \mathcal{S}^{\prime}\left(\mathbb{R}^{n}\right)$, então temos

$$
\Delta u=\Delta\left(T_{N} * f\right)=\left(\Delta T_{N}\right) * f=\delta * f=T_{f}
$$

Logo a equação de Laplace $\Delta u=f$ pode ser resolvida em $\mathcal{S}^{\prime}\left(\mathbb{R}^{n}\right)$ para toda função $f \in \mathcal{S}\left(\mathbb{R}^{n}\right)$.

Por fim, o exemplo abaixo é o de maior importância no estudo da Lei de Weyl.

Exemplo 4.39. (Equação do calor)

Seja $u:(0, \infty) \times \mathbb{R}^{n} \ni(t, x) \mapsto u(t, x) \in \mathbb{C}$ e considere o problema de valor inicial:

$$
\left\{\begin{array}{l}
u_{t}(t, x)-\Delta u(t, x)=0 \\
u(0, x)=f(x)
\end{array}\right.
$$

Este problema tem a seguinte interpretação física. Queremos estudar o fluxo de calor ao longo do tempo no espaço $\mathbb{R}^{n}$, sabendo que a temperatura inicial é dada pela função $f$. A função $u$ representa a temperatura no instante $t$ e no ponto $x$.

Assumindo que $f \in \mathcal{S}\left(\mathbb{R}^{n}\right)$ e interpretando o problema no contexto das distribuições, podemos resolvê-lo aplicando a transformada de Fourier com relação a variável $x$, obtendo a seguinte equação diferencial ordinária em $t$ :

$$
\left\{\begin{array}{l}
\hat{u}_{t}(t, \xi)+(2 \pi|\xi|)^{2} \hat{u}(t, \xi)=0 \\
\hat{u}(0, \xi)=\hat{f}(\xi)
\end{array}\right.
$$


cuja solução é

$$
\hat{u}(t, \xi)=\hat{f}(\xi) e^{-(2 \pi|\xi|)^{2} t} .
$$

Logo, utilizando a Proposição 4.6 e o Exemplo 4.12, temos

$$
\begin{aligned}
u(x, t) & =\left(f * \mathcal{F}^{-1}\left[e^{-(2 \pi|\xi|)^{2} t}\right]\right)(x) \\
& =\left(f *(4 \pi t)^{-n / 2} e^{-|x|^{2} / 4 t}\right)(x) \\
& =(4 \pi t)^{-n / 2} \int_{\mathbb{R}^{n}} f(y) e^{-|x-y|^{2} / 4 t} d y \\
& =\int_{\mathbb{R}^{n}} f(y) k_{t}(x, y) d y
\end{aligned}
$$

onde a função $k:(0, \infty) \times \mathbb{R}^{n} \times \mathbb{R}^{n} \rightarrow \mathbb{R}$ definida por

$$
k(t, x, y)=k_{t}(x, y)=(4 \pi t)^{-n / 2} e^{-|x-y|^{2} / 4 t}
$$

é chamada de núcleo do calor ou núcleo Gaussiano. 


\section{A Lei de Weyl}

De maneira informal, tendo como referência a questão levantada por Kac apresentada na introdução, a Lei de Weyl afirma que podemos escutar a área de um tambor.

$\mathrm{Na}$ época em que este problema foi proposto, o grande matemático David Hilbert declarou que acreditava que este teorema não seria demonstrado durante seu período de vida. No entanto, dois anos após essa afirmação, em 1911, seu aluno Herman Weyl solucionou a questão utilizando a recente teoria das equações integrais desenvolvida por seu professor.

\subsection{O Laplaciano em $\mathbb{R}^{n}$ e em $\Omega$, com condições de contorno de Dirichlet}

Nesta seção definimos o Laplaciano com condições de contorno de Dirichlet em espaços de Sobolev e provamos que, para domínios limitados, o operador Laplaciano é simétrico e m-dissipativo. Acrescentando o fato de que seu resolvente é compacto, poderemos aplicar o Teorema Espectral para decompor o operador Lapaciano em seus autovalores e autovetores.

Ao longo desta seção $\Omega$ denota um conjunto aberto de $\mathbb{R}^{n}$.

Definição 5.1. (Suporte)

O suporte de $f: \Omega \rightarrow \mathbb{C}$ é o conjunto $\operatorname{supp}(f):=\overline{\{x \in \Omega: f(x) \neq 0\}}$. A função $f: \Omega \rightarrow \mathbb{C}$ tem suporte compacto se $\operatorname{supp}(f)$ é um conjunto compacto contido em $\Omega$. Denotamos por $C_{c}(\Omega)=\{f \in C(\Omega): \operatorname{supp}(f)$ é compacto $\}$ o espaço das funções contínuas com suporte compacto e $C_{c}^{k}(\Omega)=C^{k}(\Omega) \cap C_{c}(\Omega)$.

No capítulo anterior estudamos as distribuições temperadas, que são os funcionais lineares contínuos que agem no espaço de Schwartz. Se nós substituirmos o espaço de Schwartz pelo espaço das funções infinitamente diferenciáveis e com suporte compacto $\mathcal{D}(\Omega):=C^{\infty}(\Omega) \cap C_{c}(\Omega)$, também chamado de espaço das funções teste, obtemos o espaço das distribuições $\mathcal{D}^{\prime}(\Omega)$.

Definição 5.2. (Convergência em $\mathcal{D}(\Omega)$ )

Dizemos que a sequência de funções $\left(f_{j}\right)_{j \in \mathbb{N}}$ em $\mathcal{D}(\Omega)$ converge para $f \in \mathcal{D}(\Omega)$, se existe um conjunto compacto $K \subset \Omega$ tal que, para todo $j \in \mathbb{N}$, $\operatorname{supp}\left(f_{j}\right) \subset K$ e, para 
todo $\alpha \in \mathbb{N}_{0}^{n}$, quando $j \rightarrow \infty$, temos a convergência uniforme

$$
\left\|\partial^{\alpha} f_{j}-\partial^{\alpha} f\right\|_{L^{\infty}(\Omega)} \rightarrow 0
$$

\section{Definição 5.3. (Distribuição)}

Uma distribuição $T \in \mathcal{D}^{\prime}(\Omega)$ é um funcional linear contínuo $T: \mathcal{D}(\Omega) \rightarrow \mathbb{C}$ no seguinte sentido: para toda sequência convergente $f_{j} \rightarrow f$ em $\mathcal{D}(\Omega)$, temos $T\left(f_{j}\right) \rightarrow T(f)$, quando $j \rightarrow \infty$.

Entre as distribuições, podemos destacar o importante espaço de Sobolev, definido abaixo.

Definição 5.4. (Espaço de Sobolev)

O espaço de Sobolev $W^{k, p}(\Omega)$ é definido por

$$
W^{k, p}(\Omega):=\left\{f \in L^{p}(\Omega): \partial^{\alpha} f \in L^{p}(\Omega), \forall|\alpha| \leq k\right\}
$$

Nesta definição, a frase $\partial^{\alpha} f \in L^{p}$ deve ser entendida no contexto das distribuições, isto é, dado $|\alpha| \leq k$, existe $g_{\alpha} \in L^{p}(\Omega)$ tal que para toda $\varphi \in C_{c}^{\infty}(\Omega)$ temos

$$
\int_{\Omega} g_{\alpha}(x) \varphi(x) d x=(-1)^{|\alpha|} \int_{\Omega} f(x) \partial^{\alpha} \varphi(x) d x .
$$

Equipado com a norma

$$
\|f\|_{W^{k, p}(\Omega)}=\sum_{|\alpha| \leq k}\left\|\partial^{\alpha} f\right\|_{L^{p}}
$$

o espaço $W^{k, p}(\Omega)$ é um espaço de Banach separável, se $p \in[1, \infty)$. O espaço $W^{k, 2}(\Omega)$ é denotado por $H^{k}(\Omega)$, pois é um espaço de Hilbert com o produto interno

$$
\langle f, g\rangle_{H^{k}(\Omega)}=\sum_{|\alpha| \leq k}\left\langle\partial^{\alpha} f, \partial^{\alpha} g\right\rangle_{L^{2}(\Omega)}
$$

\section{Definição 5.5.}

Dizemos que uma função de $H^{1}(\Omega)$ satisfaz as condições de contorno de Dirichlet no sentido fraco, se ela pertence ao espaço $H_{0}^{1}(\Omega)=\overline{\mathcal{D}(\Omega)}{ }^{H^{1}(\Omega)}$, isto é, se ela pode ser 
aproximada por funções teste. Seja $\Omega \subset \mathbb{R}^{n}$. O Laplaciano de Dirichlet é o operador

$$
\Delta_{\Omega}^{D}: D\left(\Delta_{\Omega}^{D}\right) \subset L^{2}(\Omega) \rightarrow L^{2}(\Omega)
$$

com domínio

$$
D\left(\Delta_{\Omega}^{D}\right)=\left\{f \in H_{0}^{1}(\Omega): \Delta f \in L^{2}(\Omega)\right\}
$$

onde $\Delta f$ deve ser interpretado no sentido de distribuição, isto é, existe $g \in L^{2}(\Omega)$ tal que para toda $\varphi \in \mathcal{D}(\Omega)$ temos

$$
\int_{\Omega} g(x) \varphi(x) d x=\int_{\Omega} f(x) \Delta \varphi(x) d x
$$

Assim, o Laplaciano é definido por

$$
\Delta_{\Omega}^{D}(f)=g
$$

Observe que tomando $\nabla f$ no sentido de distribuições, concluímos que $f \in D\left(\Delta_{\Omega}^{D}\right)$ se, e somente se, existe $g \in L^{2}(\Omega)$ tal que, para toda $\varphi \in \mathcal{D}(\Omega)$, temos

$$
\int_{\Omega} g(x) \varphi(x) d x=\int_{\Omega} \nabla f(x) \nabla \varphi(x) d x
$$

Enunciamos o Teorema de Representação de Riesz, que será utilizado na demonstração do próximo resultado.

Teorema 5.6. (Teorema de representação de Riesz)

Se $H$ é um espaço de Hilbert e $f \in H^{*}$, então existe um único y $\in H$ tal que $f(x)=\langle x, y\rangle$, para todo $x \in H$.

\section{Teorema 5.7.}

O operador $\Delta_{\Omega}^{D}$ é simétrico e m-dissipativo.

Demonstração. 
Seja $f \in D\left(\Delta_{\Omega}^{D}\right), \varphi \in \mathcal{D}(\Omega)$. Temos

$$
\begin{aligned}
\left\langle\Delta_{\Omega}^{D} f, \varphi\right\rangle_{L^{2}(\Omega)} & =\int_{\Omega} \Delta f \bar{\varphi} \\
& =-\int_{\Omega} \nabla f \overline{\nabla \varphi} \\
& =\int_{\Omega} f \overline{\Delta \varphi} \\
& =\left\langle f, \Delta_{\Omega}^{D} \varphi\right\rangle_{L^{2}(\Omega)} .
\end{aligned}
$$

Como $\mathcal{D}(\Omega)$ é denso em $H_{0}^{1}(\Omega)$, então podemos considerar $\varphi \in H_{0}^{1}(\Omega)$. Logo $\Delta_{\Omega}^{D}$ é simétrico e dissipativo, pois $\left\langle\Delta_{\Omega}^{D} f, \varphi\right\rangle_{L^{2}(\Omega)}=\left\langle f, \Delta_{\Omega}^{D} \varphi\right\rangle_{L^{2}(\Omega)}$ e

$$
\left\langle\Delta_{\Omega}^{D} \varphi, \varphi\right\rangle_{L^{2}(\Omega)}=-\int_{\Omega} \nabla \varphi \overline{\nabla \varphi} \leq 0
$$

Falta mostrar que $\left(I-\Delta_{\Omega}^{D}\right)$ é sobrejetora. Seja $f \in L^{2}$, então $\phi(v)=\int_{\Omega} f v$ define um funcional linear contínuo em $H_{0}^{1}$. Pelo Teorema de representação de Riesz 5.6, existe um único $u \in H_{0}^{1}$ tal que

$$
\int_{\Omega} f v=\langle v, u\rangle_{H^{1}(\Omega)}=\int_{\Omega} v \bar{u}+\int_{\Omega} \nabla v \nabla \bar{u}
$$

para todo $v \in H_{0}^{1}(\Omega)$. Em particular, para todo $v \in \mathcal{D}(\Omega)$ temos

$$
\int_{\Omega}(\bar{u}-f) v=-\int_{\Omega} \nabla \bar{u} \nabla v
$$

$\operatorname{Logo} u \in D\left(\Delta_{\Omega}^{D}\right)$ e $\Delta_{\Omega}^{D} u=u-f$.

\section{Corolário 5.8.}

Pelo Teorema 3.15, o operador $\Delta_{\Omega}^{D}$ gera um $C_{0}$-semigrupo $T$ de operadores operadores em $L^{2}(\Omega)$ autoadjuntos e contrativos, denotado por $T(t)=e^{t \Delta_{\Omega}^{D}}$. Além do mais, pelo Teorema Espectral e pela Proposição 3.6, dado $f \in D\left(\Delta_{\Omega}^{D}\right), u(t)=e^{t \Delta_{\Omega}^{D}} f$ é a única 
solução da equação do calor com condições de contorno de Dirichlet, dada por:

$$
\left\{\begin{array}{l}
u \in C^{1}\left([0, \infty) ; L^{2}(\Omega)\right) \cap C\left([0, \infty) ; D\left(\Delta_{\Omega}^{D}\right)\right. \\
u(t) \in H_{0}^{1}(\Omega), t>0 \\
u^{\prime}(t)=\Delta u(t), t>0 \\
u(0)=f
\end{array}\right.
$$

Vamos agora estudar o Laplaciano em $\mathbb{R}^{n}$. Neste caso não há fronteira e o domínio do Laplaciano é definido como $D(\Delta)=H^{2}\left(\mathbb{R}^{n}\right)$. Verificaremos, a seguir, que o Laplaciano em $\mathbb{R}^{n}$ é gerador do semigrupo Gaussiano.

\section{Proposição 5.9.}

Seja $k_{t}$ o núcleo do calor deduzido no Exemplo 4.39. O Laplaciano em $L^{2}\left(\mathbb{R}^{n}\right)$ é gerador do $C_{0}$-semigrupo Gaussiano $G:(0, \infty) \rightarrow \mathcal{L}\left(L^{2}\left(\mathbb{R}^{n}\right)\right)$ definido por

$$
(G(t) f)(x):=\left(k_{t} * f\right)(x)=(4 \pi t)^{-n / 2} \int_{\mathbb{R}^{n}} f(x-y) e^{-|y|^{2} / 4 t} d y
$$

Demonstração.

Seja $f \in L^{2}(\Omega)$, então

$$
\|G(t) f\|_{L^{2}\left(\mathbb{R}^{n}\right)}=\left\|k_{t} * f\right\| \leq\left\|k_{t}\right\|_{L^{1}\left(\mathbb{R}^{n}\right)}\|f\|_{L^{2}\left(\mathbb{R}^{n}\right)}=\|f\|_{L^{2}\left(\mathbb{R}^{n}\right)}
$$

logo $G(t)$ é limitado para todo $t>0$. Temos também $G(t+s)=G(t) G(s)$. De fato

$$
\begin{aligned}
\mathcal{F}(G(t) G(s) f) & =\mathcal{F}\left(k_{t} * k_{s} * f\right) \\
& =\hat{k}_{t} \hat{k}_{s} \hat{f} \\
& =\hat{k}_{t+s} \hat{f} .
\end{aligned}
$$

Logo

$$
\begin{aligned}
G(t) G(s) f & =\mathcal{F}^{-1}\left(\hat{k}_{t+s} \hat{f}\right) \\
& =k_{t+s} * f \\
& =G(t+s) f .
\end{aligned}
$$

Por último, quando $t \rightarrow 0$, utilizamos o Teorema da Convergência Dominada para concluir 
que $G(t)$ é fortemente contínuo, como vemos abaixo.

$$
\|G(t) f-f\|_{L^{2}\left(\mathbb{R}^{n}\right)}=\left\|\hat{k}_{t} \hat{f}-\hat{f}\right\|_{L^{2}\left(\mathbb{R}^{n}\right)}=\left\|\left(\hat{k}_{t}-1\right) \hat{f}\right\|_{L^{2}\left(\mathbb{R}^{n}\right)} \rightarrow 0 .
$$

Logo $G$ é um $C_{0}$-semigrupo. Para mostrar que seu gerador $A$ é o Laplaciano, observe que, para $f \in H^{2}\left(\mathbb{R}^{n}\right)$, temos

$$
\begin{aligned}
\frac{\left(\hat{k}_{t}(\xi)-1\right) \hat{f}(\xi)}{t} & =\frac{e^{-t|\xi|^{2}}-1}{t} \hat{f}(\xi) \\
& =\frac{1}{t} \int_{0}^{1} \frac{d}{d \theta}\left(e^{-t \theta|\xi|^{2}}\right) d \theta \hat{f}(\xi) \\
& =\int_{0}^{1} e^{-t \theta|\xi|^{2}} d \theta\left(-|\xi|^{2} \hat{f}(\xi)\right) \\
& =\int_{0}^{1} e^{-t \theta|\xi|^{2}} d \theta(\widehat{\Delta f}(\xi)) \\
& \rightarrow(\widehat{\Delta f}(\xi)),
\end{aligned}
$$

em $L^{2}\left(\mathbb{R}^{n}\right)$, quando $t \rightarrow 0$, novamente pelo Teorema da Convergência Dominada. Observe também que se $f \in L^{2}\left(\mathbb{R}^{n}\right)$ e existe o limite em $L^{2}\left(\mathbb{R}^{n}\right)$ acima, então $\Delta f \in L^{2}\left(\mathbb{R}^{n}\right)$ e, portanto, $f \in H^{2}\left(\mathbb{R}^{n}\right)$. Como $\mathcal{F}: L^{2}\left(\mathbb{R}^{n}\right) \rightarrow L^{2}\left(\mathbb{R}^{n}\right)$ é um isomorfismo, temos

$$
A f=\lim _{t \rightarrow 0} \frac{G(t) f-f}{t}=g \Longleftrightarrow \lim _{t \rightarrow 0} \frac{\left(\hat{k}_{t}-1\right) \hat{f}}{t}=\hat{g} .
$$

Logo $f \in D(A) \Longleftrightarrow f \in D(\Delta)$ e $A f=\Delta f$.

\subsection{Operadores de Hilbert-Schmidt}

Para nosso estudo, será interessante mostrar que o semigrupo gerado pelo Laplaciano de Dirichlet em domínios limitados tem propriedades mais fortes do que a compacidade. Vamos mostrar que o Laplaciano de Dirichlet em domínios limitados gera um semigrupo de operadores de Hilbert-Schmidt. Nesta seção, definimos os operadores de Hilbert-Schmidt e provamos o critério que será utilizado para mostrar que um operador pertence a esta classe. Os operadores de Hilbert-Schmidt considerados estão fazem parte de uma classe de operadores mais gerais, chamados operadores integrais.

Definição 5.10. (Operador integral)

Seja $\Omega \subset \mathbb{R}^{n}$ um aberto. Um operador $T$ em $L^{2}(\Omega)$ é um operador integral se existe 
uma função mensurável $k: \Omega \times \Omega \rightarrow \mathbb{C}$ tal que, para todo $f \in L^{2}(\Omega)$ e para quase todo $x \in \Omega$, temos

$$
(T f)(x)=\int_{\Omega} k(x, y) f(y) d y
$$

A função $k$ é chamada o núcleo de $T$.

Proposição 5.11. (Operador de Hilbert-Schmidt)

Seja $T$ um operador em $L^{2}(\Omega)$ e $\left\{e_{j} \in L^{2}(\Omega): j \in \mathbb{N}\right\}$ uma base ortonormal de $L^{2}(\Omega)$. Então as seguintes afirmações são equivalentes:

(a) $\|T\|_{H S}^{2}:=\sum_{j=1}^{\infty}\left\|T e_{j}\right\|^{2}<\infty$.

(b) T é um operador integral com núcleo $k \in L^{2}(\Omega \times \Omega)$.

O operador T é chamado de Hilbert-Schmidt se satisfaz essas afirmações. Neste caso $\|T\|_{H S}=\|k\|_{L^{2}(\Omega \times \Omega)}$.

Demonstração.

(b) $\Longrightarrow\left(\right.$ a) Se $\left\{e_{n}: n \in \mathbb{N}\right\}$ é uma base ortonormal de $L^{2}(\Omega)$, então $\left\{\overline{e_{n}} \otimes e_{j}: n, j \in \mathbb{N}\right\}$ é uma base ortonormal de $L^{2}(\Omega \times \Omega)$, logo, utilizando a identidade de Parseval, temos

$$
\begin{aligned}
\sum_{n=1}^{\infty}\left\|T e_{n}\right\|_{L^{2}(\Omega)}^{2} & =\sum_{n=1}^{\infty} \sum_{j=1}^{\infty}\left|\left\langle T e_{n}, e_{j}\right\rangle_{L^{2}(\Omega)}\right|^{2} \\
& =\sum_{n=1}^{\infty} \sum_{j=1}^{\infty}\left|\left\langle k, e_{j} \otimes \overline{e_{n}}\right\rangle_{L^{2}(\Omega \times \Omega)}\right|^{2} \\
& =\|k\|_{L^{2}(\Omega \times \Omega)}^{2}<\infty .
\end{aligned}
$$

$(\mathrm{a}) \Longrightarrow(\mathrm{b})$ Seja $k(x, y):=\sum_{n=1}^{\infty} \sum_{j=1}^{\infty}\left\langle T e_{n}, e_{j}\right\rangle_{L^{2}(\Omega)} \overline{e_{n}(x)} e_{j}(y)$ e $T_{k}$ o operador integral com núcleo $k$. Note que $\|k\|_{L^{2}(\Omega \times \Omega)}=\|T\|_{H S}$. Seja $f, g \in L^{2}(\Omega)$. Usando que $T$ é limitado 
e a continuidade do produto interno, temos:

$$
\begin{aligned}
\left\langle T_{k} f, g\right\rangle_{L^{2}(\Omega)} & =\int_{\Omega} \int_{\Omega} k(x, y) f(y) \overline{g(x)} d x d y \\
& =\langle k, g \otimes \bar{f}\rangle_{L^{2}(\Omega \times \Omega)} \\
& =\sum_{n=1}^{\infty} \sum_{j=1}^{\infty}\left\langle T e_{n}, e_{j}\right\rangle_{L^{2}(\Omega)}\left\langle\overline{e_{n}} \otimes e_{j}, \bar{f} \otimes g\right\rangle_{L^{2}(\Omega \times \Omega)} \\
& =\sum_{n=1}^{\infty} \sum_{j=1}^{\infty}\left\langle T e_{n}, e_{j}\right\rangle_{L^{2}(\Omega)}\left\langle f, e_{n}\right\rangle_{L^{2}(\Omega)}\left\langle e_{j}, g\right\rangle_{L^{2}(\Omega)} \\
& =\left\langle\sum_{n=1}^{\infty}\left\langle f, e_{n}\right\rangle_{L^{2}(\Omega)} T e_{n}, \sum_{j=1}^{\infty}\left\langle g, e_{j}\right\rangle_{L^{2}(\Omega)} e_{j}\right\rangle_{L^{2}(\Omega)} \\
& =\left\langle\sum_{n=1}^{\infty}\left\langle f, e_{n}\right\rangle_{L^{2}(\Omega)} T e_{n}, g\right\rangle_{L^{2}(\Omega)} \\
& =\left\langle T \sum_{n=1}^{\infty}\left\langle f, e_{n}\right\rangle_{L^{2}(\Omega)} e_{n}, g\right\rangle_{L^{2}(\Omega)} \\
& =\langle T f, g\rangle_{L^{2}(\Omega)} .
\end{aligned}
$$

Logo $T$ é um operador integral com núcleo $k$.

É um fato conhecido que todo operador de Hilbert-Schmidt é compacto. Abaixo segue um critério importante para que um operador seja de Hilbert-Schmidt.

Proposição 5.12. (Condição para um operador ser de Hilbert-Schmidt)

Seja $\Omega \subset \mathbb{R}^{n}$ um conjunto de medida finita. Se $T \in \mathcal{L}\left(L^{2}(\Omega)\right)$ é um operador tal que $T\left(L^{2}(\Omega)\right) \subset L^{\infty}(\Omega)$, então $T$ é um operador de Hilbert-Schmidt.

Demonstração.

Observe que se $T \in \mathcal{L}\left(L^{2}(\Omega)\right)$ e $T\left(L^{2}(\Omega)\right) \subset L^{\infty}(\Omega)$, então $T: L^{2}(\Omega) \rightarrow L^{\infty}(\Omega)$ é contínuo pelo Teorema do Gráfico Fechado. Seja $d \in \mathbb{N}, B$ a bola unitária fechada em $\mathbb{R}^{d}$ e $\left\{e_{j} \in L^{2}(\Omega): j \in \mathbb{N}\right\}$ uma base ortonormal de $L^{2}(\Omega)$. Note que para $y=\left(y_{1}, y_{2}, \ldots, y_{d}\right) \in$ 
$B$, temos

$$
\begin{aligned}
\left\|\sum_{j=1}^{d} y_{j} e_{j}\right\|_{L^{2}(\Omega)}^{2} & =\int_{\Omega}\left|\sum_{j=1}^{d} y_{j} e_{j}(x)\right|^{2} d x \\
& \leq \sum_{j=1}^{d} y_{j}^{2} \int_{\Omega}\left|e_{j}(x)\right|^{2} d x \\
& =\sum_{j=1}^{d} y_{j}^{2} \leq 1 .
\end{aligned}
$$

Logo, para todo $y \in B$ e para quase todo $x \in \Omega$, temos

$$
\left|\sum_{j=1}^{d} y_{j}\left(T e_{j}\right)(x)\right|=\left|\left(T\left(\sum_{j=1}^{d} y_{j} e_{j}\right)\right)(x)\right| \leq\|T\|_{\mathcal{L}\left(L^{2}(\Omega), L^{\infty}(\Omega)\right)} .
$$

Note que para $\alpha, \beta \in \mathbb{R}^{d}$,

$$
\sup _{\|\alpha\| \leq 1}\left|\langle\alpha, \beta\rangle_{\mathbb{R}^{d}}\right|=\|\beta\|_{\mathbb{R}^{d}}
$$

Logo, para quase todo $x \in \Omega$, temos

$$
\left|\sup _{y \in B} \sum_{j=1}^{d} y_{j}\left(T e_{j}\right)(x)\right|=\sqrt{\sum_{j=1}^{d}\left|\left(T e_{j}\right)(x)\right|^{2}} .
$$

Substituindo (13) em (12) temos

$$
\sum_{j=1}^{d}\left|\left(T e_{j}\right)(x)\right|^{2} \leq\|T\|^{2}
$$

Por fim, integrando sobre $\Omega$, temos

$$
\sum_{j=1}^{d}\left\|T e_{j}\right\|_{L^{2}(\Omega)}^{2} \leq|\Omega|\|T\|^{2}<\infty
$$

A equação acima vale para todo $d \in \mathbb{N}$, logo o operador $T$ é de Hilbert-Schmidt pela Proposição 5.11. 


\subsection{Monotonicidade}

Utilizaremos o semigrupo gerado pelo Laplaciano de Dirichlet em $\mathbb{R}^{n}$ como um limitante do semigrupo gerado pelo Laplaciano de Dirichlet em um domínio limitado $\Omega$ de $\mathbb{R}^{n}$. Isso permite mostrar que, para todo $t>0$, o operador $e^{t \Delta_{\Omega}^{D}}$ é de Hilbert-Schmidt com núcleo $k$ limitado pelo núcleo do calor.

Nesse sentido, primeiro definimos a noção de positividade que estabelece uma ordem e permite comparar funções, operadores e semigrupos.

Definição 5.13. (Positividade)

Seja $p \in[1, \infty]$. Dizemos que uma função $f \in L^{p}(\Omega)$ é positiva e denotamos $f \geq 0$ se $f(x) \geq 0$, para quase todo $x \in \Omega$. Definimos

$$
L^{p}(\Omega)_{+}=\left\{f \in L^{p}(\Omega): f \geq 0\right\}
$$

Dizemos que o operador contínuo $T: L^{p}(\Omega) \rightarrow L^{q}(\Omega)$ é positivo e denotamos $T \geq 0$, se $T(f) \geq 0$, para toda $f \geq 0$. Dados dois operadores contínuos positivos $T$ e $S$, dizemos que $T \geq S$ se, e somente se, $T-S \geq 0$. Analogamente, $T \geq S$ em $\mathcal{D}^{\prime}(\Omega)$ se, e somente se, $T(\varphi) \geq S(\varphi)$, para toda $\varphi \in \mathcal{D}(\Omega)_{+}:=\left\{\varphi \in C_{c}^{\infty}(\Omega): \varphi \geq 0\right\}$.

Compararemos funções em domínios diferentes. Por isso, para evitar confusão, a seguinte observação é importante.

\section{Observação 5.14 .}

Seja $\Omega_{1} \subset \Omega_{2} \subset \mathbb{R}^{n}, f \in L^{p}\left(\Omega_{1}\right)$ e $g \in L^{p}\left(\Omega_{2}\right)$. Neste caso podemos considerar $g$ como um membro de $L^{p}\left(\Omega_{1}\right)$ tomando sua restrição $\left.g\right|_{\Omega_{1}}$. O mesmo raciocínio é feito para os operadores $A \in \mathcal{L}\left(L^{p}\left(\Omega_{1}\right)\right)$ e $B \in \mathcal{L}\left(L^{p}\left(\Omega_{2}\right)\right)$. Podemos enxergar o $B$ como um membro de $\mathcal{L}\left(L^{p}\left(\Omega_{1}\right)\right)$ fazendo $\left.B\right|_{\Omega_{1}}(f):=\left.(B(\tilde{f}))\right|_{\Omega_{1}}$, onde $\tilde{f}$ é a extensão de $f$ em $\Omega_{2}$ com valor zero em $\Omega_{2} \backslash \Omega_{1}$. Para os semigrupos $T$ em $L^{p}\left(\Omega_{1}\right)$ e $S$ em $L^{p}\left(\Omega_{2}\right), S$ é visto como um semigrupo em $L^{p}\left(\Omega_{1}\right)$ fazendo $S(t)=\left.(S(t))\right|_{\Omega_{1}}$. Assim, expressões da forma $f \leq g, A \leq B$ e $T \leq S$ fazem sentido e serão utilizadas com este significado subentendido.

Em [2], o teorema abaixo é demonstrado como um corolário do Critério de DunfordPettis para núcleos, um resultado mais geral que mostra uma condição suficiente para um operador ser integral com núcleo $k$. Para domínios de medida finita, o mesmo resultado pode ser obtido utilizando a Proposição 5.12. É o que fazemos a seguir. 


\section{Teorema 5.15.}

Sejam $\Omega_{1}, \Omega_{2} \subset \mathbb{R}^{n}$ abertos, $\Omega_{1} \subset \Omega_{2},\left|\Omega_{1}\right|<\infty, T_{1} \in \mathcal{L}\left(L^{2}\left(\Omega_{1}\right)\right)$ e $T_{2} \in \mathcal{L}\left(L^{2}\left(\Omega_{2}\right)\right)$ tal que $0 \leq T_{1} \leq T_{2}$. Se $T_{2}$ é um operador integral com núcleo limitado $k_{2}$, então $T_{1}$ é um operador de Hilbert-Schmidt com núcleo limitado $k_{1}$ e $0 \leq k_{1} \leq k_{2}$.

Demonstração.

Vamos mostrar que $T_{1}$ satisfaz a condição da Proposição 5.12: $T_{1}\left(L^{2}(\Omega)\right) \subset L^{\infty}(\Omega)$. Seja $f \in L^{2}(\Omega)$, logo

$$
f=(\operatorname{Re} f)_{+}-(\operatorname{Re} f)_{-}+i(\operatorname{im} f)_{+}-i(\operatorname{Im} f)_{-} .
$$

Assim, basta considerar $f \in L^{2}(\Omega)_{+}$. Para quase todo $x \in \Omega$, temos

$$
\begin{aligned}
\left|\left(T_{1} f\right)(x)\right| & \leq\left|\left(T_{2} f\right)(x)\right| \\
& =\left|\int_{\Omega} k_{2}(x, y) f(y) d y\right| \\
& \leq\left(\int_{\Omega}\left|k_{2}(x, y)\right|^{2} d y\right)^{1 / 2}\left(\int_{\Omega}|f(y)|^{2} d y\right)^{1 / 2} \\
& \leq\left|k_{2}\right|_{L^{\infty}(\Omega \times \Omega)}|\Omega|^{1 / 2}\|f\|_{L^{2}(\Omega)} \\
& <\infty .
\end{aligned}
$$

Logo $T_{1}$ é um operador de Hilbert-Schmidt com núcleo $k_{1} \in L^{2}(\Omega \times \Omega)$. Suponha, por absurdo, que $k_{1}<0$ ou $k_{1}>k_{2}$. Então existem conjuntos $E, F \subset \Omega$ de medida não nula tal que para todo $(x, y) \in E \times F, k_{1}(x, y)>k_{2}(x, y)$ ou $k_{1}(x, y)<0$. Neste caso seja $f=1_{F}$. Então para todo $x \in E$ temos

$$
\begin{aligned}
T_{1}(f)(x) & =\int_{\Omega} k_{1}(x, y) f(y) d y \\
& =\int_{F} k_{1}(x, y) d y \\
& >\int_{F} k_{2}(x, y) d y \\
& =\int_{\Omega} k_{2}(x, y) f(y) d y \\
& =T_{2}(f)(x)
\end{aligned}
$$


ou

$$
\begin{aligned}
T_{1}(f)(x) & =\int_{\Omega} k_{1}(x, y) f(y) d y \\
& =\int_{F} k_{1}(x, y) d y \\
& <0 .
\end{aligned}
$$

Os dois casos contrariam a hipótese $0 \leq T_{1} \leq T_{2}$. Logo $0 \leq k_{1} \leq k_{2}$ e $k_{1} \in L^{\infty}(\Omega \times \Omega)$.

Agora passamos à tarefa de mostrar que o semigrupo $e^{t \Delta_{\Omega}^{D}}$ gerado pelo Laplaciano de Dirichlet $\Delta_{\Omega}^{D}$ possui um núcleo que é monotônico com relação a $\Omega$.

\section{Lema 5.16.}

$\operatorname{Sejam} \mathcal{D}(\Omega)_{+}:=\{\varphi \in \mathcal{D}(\Omega): \varphi \geq 0\}$ e $H_{0}^{1}(\Omega)_{+}:=L^{2}(\Omega)_{+} \cap H_{0}^{1}(\Omega)$. Então $\mathcal{D}(\Omega)_{+}$é denso em $H_{0}^{1}(\Omega)_{+}$.

\section{Demonstração.}

Seja $f \in H_{0}^{1}(\Omega)_{+}$. Então existe uma sequência de funções com valores reais $\left(\varphi_{k}\right)_{k \in \mathbb{N}}$ em $\mathcal{D}(\Omega)$ tal que $\varphi_{k} \rightarrow f \in H^{1}(\Omega)$. Seja $f^{+}(x):=\max (f(x), 0)$. A função $f \mapsto f^{+}$é contínua de $H^{1}(\Omega)$ em $H^{1}(\Omega)$ (Ver exercício 17 do capítulo 5 de [17]). $\operatorname{Logo} \varphi_{k}^{+} \rightarrow f^{+}=f$ em $H^{1}(\Omega)$. Para todo $k \in \mathbb{N}, \varphi_{k}^{+}$pode ser aproximada por funções teste positivas, já que $\varphi_{k}^{+}$tem suporte compacto. De fato, isto pode ser feito pela convolução com um molificador.

\section{Lema 5.17.}

Sejam $\lambda>0, u \in H_{0}^{1}(\Omega)$ e $v \in H^{1}(\Omega)$ com valores reais, $v \geq 0$. Se $\lambda u-\Delta u \leq \lambda v-\Delta v$ em $\mathcal{D}^{\prime}(\Omega)$, então $u \leq v$.

\section{Demonstração.}

Para toda $\varphi \in \mathcal{D}(\Omega)_{+}$, temos

$$
\int_{\Omega} \lambda u \varphi d x+\int_{\Omega} \nabla u \nabla \varphi d x \leq \int_{\Omega} \lambda v \varphi d x+\int_{\Omega} \nabla v \nabla \varphi d x
$$

Pelo Lema 5.16, podemos considerar $\varphi \in H_{0}^{1}(\Omega)_{+}$. Temos $(u-v)^{+} \in H_{0}^{1}(\Omega)_{+}$. De fato, existe uma sequência $\left(u_{k}\right)_{k \in \mathbb{N}}$ em $\mathcal{D}(\Omega)$ tal que $u_{k} \rightarrow u \in H^{1}(\Omega)$. Como $\left(u_{k}-v\right)^{+}$tem 
suporte compacto pois $v \geq 0$, então $\left(u_{k}-v\right)^{+} \in H_{0}^{1}(\Omega)$. Fazendo $\varphi=(u-v)^{+}$, temos

$$
\int_{\Omega} \lambda u(u-v)^{+} d x+\int_{\Omega} \nabla u \nabla(u-v)^{+} d x \leq \int_{\Omega} \lambda v(u-v)^{+} d x+\int_{\Omega} \nabla v \nabla(u-v)^{+} d x
$$

ou seja,

$$
\int_{\Omega} \lambda(u-v)(u-v)^{+} d x \leq \int_{\Omega} \nabla(v-u) \nabla(u-v)^{+} d x .
$$

Logo

$$
\begin{aligned}
\int_{\Omega} \lambda(u-v)^{+}(u-v)^{+} d x & =\int_{\Omega} \lambda(u-v)(u-v)^{+} d x \\
& \leq \int_{\Omega} \nabla(v-u) \nabla(u-v)^{+} d x \\
& =-\int_{\Omega}\left|\nabla(v-u)^{+}\right|^{2} d x \\
& \leq 0
\end{aligned}
$$

Portanto, $(u-v)^{+} \leq 0$. Então $(u-v)^{+}=0$ e $u \leq v$.

\section{Teorema 5.18.}

Sejam $\Omega_{1}, \Omega_{2} \subset \mathbb{R}^{n}$ abertos. Se $\Omega_{1} \subset \Omega_{2}$, então $0 \leq e^{t \Delta_{\Omega_{1}}^{D}} \leq e^{t \Delta_{\Omega_{2}}^{D}}$.

\section{Demonstração.}

Sejam $\lambda>0, f \in L^{2}\left(\Omega_{1}\right), f \leq 0$. Como

$$
e^{t \Delta_{\Omega}^{D}}=\lim _{n \rightarrow \infty}\left(I-\frac{t}{n} \Delta_{\Omega}^{D}\right)^{-n}
$$

na (ver Corolário 2.5 de [16]), é suficiente mostrar que

$$
u=R\left(\lambda, \Delta_{\Omega_{1}}^{D}\right) f \leq R\left(\lambda, \Delta_{\Omega_{2}}^{D}\right) f=v
$$

Como $u \in H_{0}^{1}\left(\Omega_{1}\right)_{+},\left.v\right|_{\Omega_{1}} \in H^{1}\left(\Omega_{1}\right)$ e $\lambda u-\Delta u=f=\lambda v-\Delta v$, segue do Lema 5.17 que $u \leq v$.

Como consequência temos que, para todo aberto $\Omega \subset \mathbb{R}^{n}$ e $t>0$, o operador $e^{t \Delta_{\Omega}^{D}}$ possui um núcleo $k_{t}$ que é limitado pelo núcleo do calor. 


\section{Teorema 5.19.}

Para todo aberto $\Omega \subset \mathbb{R}^{n}$ e $t>0$, $e^{t \Delta_{\Omega}^{D}}$ possui um núcleo $k_{t}$ que satisfaz

$$
0 \leq k_{t}(x, y) \leq(4 \pi t)^{-n / 2} e^{-|x-y|^{2} / 4 t}
$$

Demonstração.

Segue do Teorema 5.18 que $0 \leq e^{t \Delta_{\Omega}^{D}} \leq G(t)$. Como $G(t)$ tem núcleo limitado $(4 \pi t)^{-n / 2} e^{-|x-y|^{2} / 4 t}$, o resultado segue do Teorema 5.15.

Por fim, mostramos que $\Delta_{\Omega}^{D}$ tem resolvente compacto.

\section{Corolário 5.20.}

Se $\Omega \subset \mathbb{R}^{n}$ é um aberto de medida finita, então $e^{t \Delta_{\Omega}^{D}}$ é compacto para todo $t>0$. Consequentemente, $\Delta_{\Omega}^{D}$ tem resolvente compacto.

Demonstração.

Pelo Teorema 5.19, o núcleo $k_{t}$ de $e^{t \Delta_{\Omega}^{D}}$ é limitado e como $\Omega$ é um aberto de medida

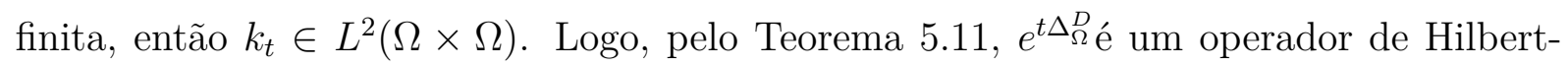
Schmidt. Consequentemente, para todo $t>0, e^{t \Delta_{\Omega}^{D}}$ é compacto. Assim podemos aplicar a Proposição 3.14 e concluir que $\Delta_{\Omega}^{D}$ tem resolvente compacto.

\subsection{Teorema de Karamata}

Veremos que, após uma certa preparação, a Lei de Weyl é consequência direta do Teorema de Karamata, que é demonstrado a seguir. Seguimos a demonstração feita em $[32]$.

\section{Teorema 5.21.}

Seja $\left(\lambda_{j}\right)_{j \in \mathbb{N}}$ uma sequência não-decrescente em $(0, \infty)$ tal que

$$
\sum_{j=1}^{\infty} e^{-\lambda_{j} t}<\infty
$$

para todo $t>0$, e defina $N(\lambda)=\#\left\{\lambda_{j} \leq \lambda\right\}$. Dado $\alpha \geq 0$ e $A \in \mathbb{R}$, as seguintes afirmações são equivalentes:

(a) $\lim _{t \rightarrow 0^{+}} t^{\alpha} \sum_{j=1}^{\infty} e^{-\lambda_{j} t}=A$; 
(b) $\lim _{\lambda \rightarrow \infty} \lambda^{-\alpha} N(\lambda)=\frac{A}{\Gamma(\alpha+1)}$.

\section{Demonstração.}

Para a demonstração do Teorema de Weyl utilizaremos apenas a implicação $(a) \Longrightarrow$ (b), por isso não demonstraremos a implicação $(b) \Longrightarrow(a)$.

$(a) \Longrightarrow(b)$ Seja $f:[0,1] \rightarrow \mathbb{R}$ uma função contínua e defina

$$
w_{f}(t):=\sum_{j=0}^{\infty} f\left(e^{-\lambda_{j} t}\right) e^{-\lambda_{j} t}
$$

e

$$
I_{f}:=\frac{A}{\Gamma(\alpha)} \int_{0}^{\infty} f\left(e^{-s}\right) s^{\alpha-1} e^{-s} d s
$$

Provaremos que

$$
\lim _{t \rightarrow 0^{+}} t^{\alpha} w_{f}(t)=I_{f}
$$

Pelo teorema de Stone-Weierstrass, é suficiente mostrar este resultado quando $f$ é um monômio da forma $f(x)=x^{m}$. Neste caso, pela hipótese, temos

$$
\begin{aligned}
\lim _{t \rightarrow 0^{+}} t^{\alpha} w_{f}(t) & =\lim _{t \rightarrow 0^{+}} t^{\alpha} \sum_{j=0}^{\infty}\left(e^{-\lambda_{j} t}\right)^{m} e^{-\lambda_{j} t} \\
& =\lim _{t \rightarrow 0^{+}} t^{\alpha} \sum_{j=0}^{\infty} e^{-\lambda_{j}(m+1) t} \\
& =\frac{A}{(m+1)^{\alpha}}
\end{aligned}
$$

Por outro lado, fazendo a mudança de variável $(m+1) s=y$, temos

$$
\begin{aligned}
I_{f}(t) & =\frac{A}{\Gamma(\alpha)} \int_{0}^{\infty}\left(e^{-s}\right)^{m} s^{\alpha-1} e^{-s} d s \\
& =\frac{A}{\Gamma(\alpha)} \int_{0}^{\infty} e^{-(m+1) s} s^{\alpha-1} d s \\
& =\frac{A}{\Gamma(\alpha)} \int_{0}^{\infty} e^{-y} y^{\alpha-1}(m+1)^{-\alpha} d y \\
& =\frac{A}{(m+1)^{\alpha}} .
\end{aligned}
$$

Para $0<r<1$, seja $f_{r}[0,1] \rightarrow \mathbb{R}$ a função contínua definida por 


$$
f_{r}(t)= \begin{cases}0, & 0 \leq t \leq r / e \\ \text { linear, } & r / e<t \leq 1 / e \\ 1 / t, & t>1 / e\end{cases}
$$

Note que

$$
\begin{aligned}
\lim _{r \rightarrow 1^{-}} I_{f_{r}} & =\lim _{r \rightarrow 1^{-}} \frac{A}{\Gamma(\alpha)} \int_{0}^{\infty} f_{r}\left(e^{-s}\right) s^{\alpha-1} e^{-s} d s \\
& =\frac{A}{\Gamma(\alpha)} \int_{0}^{1} s^{\alpha-1} d s \\
& =\frac{A}{\alpha \Gamma(\alpha)} \\
& =\frac{A}{\Gamma(\alpha+1)} .
\end{aligned}
$$

Mostrarermos que

$$
w_{f_{r}}((1-\log r) / \lambda) \leq N(\lambda) \leq w_{f_{r}}(1 / \lambda)
$$

De fato, por um lado, como $e^{-\lambda_{j}(1-\log r) / \lambda} \geq r / e$ implica $\lambda_{j} \leq \lambda$, temos

$$
\begin{aligned}
w_{f_{r}}((1-\log r) / \lambda) & =\sum_{j=0}^{\infty} f_{r}\left(e^{-\lambda_{j}(1-\log r) / \lambda}\right) e^{-\lambda_{j}(1-\log r) / \lambda} \\
& =\sum_{\lambda_{j} \leq \lambda} f_{r}\left(e^{-\lambda_{j}(1-\log r) / \lambda}\right) e^{-\lambda_{j}(1-\log r) / \lambda} \\
& \leq \sum_{\lambda_{j} \leq \lambda} 1 \\
& =N(\lambda) .
\end{aligned}
$$

Por outro lado, como $e^{-\lambda_{j} / \lambda} \geq 1 / e \Longleftrightarrow \lambda_{j} \leq \lambda$, temos

$$
\begin{aligned}
w_{f_{r}}(1 / \lambda) & =\sum_{j=0}^{\infty} f_{r}\left(e^{-\lambda_{j} / \lambda}\right) e^{-\lambda_{j} / \lambda} \\
& \geq \sum_{\lambda_{j} \leq \lambda} f_{r}\left(e^{-\lambda_{j} / \lambda}\right) e^{-\lambda_{j} / \lambda} \\
& =\sum_{\lambda_{j} \leq \lambda} 1 \\
& =N(\lambda) .
\end{aligned}
$$


Fazendo $\lambda \rightarrow \infty$ e utilizando (14) verificamos que

$$
\begin{aligned}
\limsup _{\lambda \rightarrow \infty} \lambda^{-\alpha} N(\lambda) & \leq \lim _{\lambda \rightarrow \infty} \lambda^{-\alpha} w_{f_{r}}(1 / \lambda) \\
& =I_{f_{r}}
\end{aligned}
$$

e

$$
\begin{aligned}
\liminf _{\lambda \rightarrow \infty} \lambda^{-\alpha} N(\lambda) & \geq \lim _{\lambda \rightarrow \infty} \lambda^{-\alpha} w_{f_{r}}((1-\log r) / \lambda) \\
& =(1-\log r)^{-\alpha} I_{f_{r}} .
\end{aligned}
$$

Fazendo $r \rightarrow 1^{-}$e usando (15) temos o resultado.

\subsection{Teorema de Weyl}

Agora que desenvolvemos as ferramentas necessárias, estamos em condições de demonstrar o teorema de Weyl. Por um lado, vamos mostrar que podemos escrever o núcleo do calor por meio dos autovalores e autovetores do Laplaciano. Por outro lado, vamos estabelecer uma estimativa para a área do domínio $|\Omega|$ por meio do núcleo do calor. Juntando os dois resultados, obtemos uma estimativa para a área do domínio $|\Omega|$ por meio dos autovalores do Laplaciano. Por fim, obtemos a Lei de Weyl aplicando o Teorema de Karamata.

Seja $\Omega \subset \mathbb{R}^{n}$ um domínio limitado. Pelo Teorema 5.7 e Corolário 5.20, o Laplaciano com condições de Dirichlet $\Delta_{\Omega}^{D}: L^{2}(\Omega) \rightarrow L^{2}(\Omega)$ é simétrico, m-dissipativo e tem resolvente compacto. Aplicando o Teorema Espectral 2.50, denotemos $0<\lambda_{1} \leq \lambda_{2} \leq \ldots$ os autovalores de $-\Delta_{\Omega}^{D}$ associados aos autovetores $e_{1}, e_{2}, \ldots \in L^{2}(\Omega)$.

Primeiro vamos mostrar que o núcleo $k_{t}^{\Omega}$ é uma função contínua e limitada e pode ser expresso por meio dos autovalores e autovetores do Laplaciano com condições de Dirichlet $-\Delta_{\Omega}^{D}$

\section{Proposição 5.22.}

Para todo $t>0$, temos

$$
\sum_{j=1}^{\infty} e^{-\lambda_{j} t}<\infty
$$

Demonstração. 
Seja $t>0$. Pelo Teorema 5.15, $e^{\Delta_{\Omega}^{D} t}$ é um operador de Hilbert-Schmidt. Logo

$$
\sum_{j=1}^{\infty} e^{-2 \lambda_{j} t} \leq \sum_{j=1}^{\infty}\left|e^{-\lambda_{j} t}\right|^{2}<\infty
$$

Substituindo $t$ por $t / 2$ obtemos o resultado.

\section{Lema 5.23.}

Existe $C>0$ tal que, para todo $j \in \mathbb{N}$ e para todo $x \in \Omega$, temos

$$
\left|e_{j}(x)\right| \leq C \lambda_{j}^{n / 4}
$$

Demonstração.

Seja $j \in \mathbb{N}$ e $x \in \Omega$. Pelo Teorema 5.19 e utilizando a desigualdade de Cauchy-Schwarz, para todo $t>0$, temos

$$
\begin{aligned}
\left|e^{-\lambda_{j} t} e_{j}(x)\right| & =\left|\left(e^{\Delta_{\Omega}^{D} t} e_{j}\right)(x)\right| \\
& =\left|\int_{\Omega} k_{t}(x, y) e_{j}(y) d y\right| \\
& \leq\left(\int_{\Omega}\left|(4 \pi t)^{-n / 2} e^{-|x-y|^{2} / 4 t}\right|^{2} d y\right)^{1 / 2}\left\|e_{j}\right\|_{L^{2}(\Omega)} \\
& \leq\left((4 \pi t)^{-n} \int_{\mathbb{R}^{n}} e^{-|x|^{2} / 2 t} d x\right)^{1 / 2} \\
& =2^{-3 n / 4} \pi^{-n / 4} t^{-n / 4} .
\end{aligned}
$$

Logo

$$
\left|e_{j}(x)\right| \leq 2^{-3 n / 4} \pi^{-n / 4} t^{-n / 4} e^{\lambda_{j} t}
$$

Fazendo $t=\lambda_{j}^{-1}$ basta tomar $C=2^{-3 n / 4} \pi^{-n / 4} e$.

Para o próximo resultado vamos utilizar um teorema de regularidade. Para tanto, vamos recordar a definição de conjunto de classe $C^{m}$.

Definição 5.24. (Conjunto de classe $C^{m}$ )

Um conjunto aberto $\Omega \subset \mathbb{R}^{n}$ é de classe $C^{m}$ se para todo $x \in \partial \Omega$ existe uma vizinhança $U$ de $x$ e um $C^{m}$-difeomorfismo $\phi: U \rightarrow B(0,1) \subset \mathbb{R}^{n}$ tal que:

1. $\phi(x)=0$ 
2. $\phi(U \cap \Omega)=B(0,1) \cap\left\{x \in \mathbb{R}^{n}: x_{n}>0\right\}$

3. $\phi(U \cap \partial \Omega)=B(0,1) \cap\left(\mathbb{R}^{n-1} \times\{0\}\right)$

Caso $m=\infty$, então dizemos que $\Omega$ é suave.

\section{Proposição 5.25.}

Se $\Omega$ é suave, então $e_{j} \in C^{\infty}(\bar{\Omega})$, para todo $j \in \mathbb{N}$.

Demonstração.

É consequência direta do Teorema 6 (Diferenciabilidade infinita até a fronteira) de [17, p. 326].

\section{Proposição 5.26.}

Seja $t>0$, então a série

$$
\sum_{j=1}^{\infty} e^{-\lambda_{j} t} e_{j}(x) \overline{e_{j}(y)}
$$

converge absolutamente e uniformemente para todo $(x, y) \in \bar{\Omega} \times \bar{\Omega}$.

\section{Demonstração.}

Utilizando a desigualdade de Cauchy-Schwarz, para todo $(x, y) \in \bar{\Omega} \times \bar{\Omega}$, temos

$$
\sum_{j=1}^{\infty}\left|e^{-\lambda_{j} t} e_{j}(x) \overline{e_{j}(y)}\right| \leq\left(\sum_{j=1}^{\infty} e^{-\lambda_{j} t}\left|e_{j}(x)\right|^{2}\right)^{1 / 2}\left(\sum_{j=1}^{\infty} e^{-\lambda_{j} t}\left|e_{j}(y)\right|^{2}\right)^{1 / 2}
$$

Por sua vez, utilizando o Lema 5.23, existe $C>0$ tal que, para todo $x \in \Omega$ e para todo $j \in \mathbb{N}$, temos

$$
\begin{aligned}
e^{-\lambda_{j} t}\left|e_{j}(x)\right|^{2} & \leq e^{-\lambda_{j} t} C \lambda_{j}^{n / 2} \\
& =C \lambda_{j}^{n / 2} e^{-\lambda_{j} t / 2} e^{-\lambda_{j} t / 2}
\end{aligned}
$$

O resultado segue pelo critério de convergência de Weierstrass, já que $C \lambda_{j}^{n / 2} e^{-\lambda_{j} t / 2}$ é limitado para todo $j \in \mathbb{N}$ e, pela Proposição 5.22, temos

$$
\sum_{j=1}^{\infty} e^{-\lambda_{j} t / 2}<\infty
$$




\section{Corolário 5.27.}

A série

$$
\sum_{j=1}^{\infty} e^{-\lambda_{j} t} e_{j}(x) \overline{e_{j}(y)}
$$

define uma função contínua e limitada em $\bar{\Omega} \times \bar{\Omega}$.

\section{Demonstração.}

Pela Proposição 5.25, para todo $j \in \mathbb{N}, e_{j}$ é uma função contínua em $\bar{\Omega}$. Logo a série é o limite uniforme de uma sequência de funções contínuas em $\bar{\Omega}$.

\section{Proposição 5.28.}

$$
k_{t}(x, y)=\sum_{j=1}^{\infty} e^{-\lambda_{j} t} e_{j}(x) \overline{e_{j}(y)}
$$

Demonstração.

Os autovetores $\left\{e_{j} \in L^{2}(\Omega): j \in \mathbb{N}\right\}$ formam uma base ortonormal de $L^{2}(\Omega)$. Logo $\left\{e_{j} \otimes \overline{e_{k}} \in L^{2}(\Omega \times \Omega): j, k \in \mathbb{N}\right\}$ é uma base ortonormal de $L^{2}(\Omega \times \Omega)$. Vamos mostrar que

$$
\left\langle k_{t}, e_{j} \otimes \overline{e_{k}}\right\rangle_{L^{2}(\Omega \times \Omega)}=\left\langle\sum_{l=1}^{\infty} e^{-\lambda_{l} t} e_{l}(x) \overline{e_{l}(y)}, e_{j} \otimes \overline{e_{k}}\right\rangle_{L^{2}(\Omega \times \Omega)},
$$

para todo $j, k \in \mathbb{N}$. De fato, seja $j, k \in \mathbb{N}$. Por um lado

$$
\begin{aligned}
\left\langle k_{t}, e_{j} \otimes \overline{e_{k}}\right\rangle & =\int_{\Omega} \int_{\Omega} k_{t}(x, y) \overline{e_{j}(x)} e_{k}(y) d y d x \\
& =\int_{\Omega} e^{\Delta_{\Omega}^{D} t} \overline{e_{k}(x)} e_{j}(x) d x \\
& =\int_{\Omega} e^{-\lambda_{k} t} \overline{e_{k}(x)} e_{j}(x) d x \\
& =e^{-\lambda_{k} t} \delta_{j k} .
\end{aligned}
$$

Por outro lado,

$$
\begin{aligned}
\left\langle\sum_{l=1}^{\infty} e^{-\lambda_{l} t} e_{l}(x) \overline{e_{l}(y)}, e_{j} \otimes \overline{e_{k}}\right\rangle & =\int_{\Omega} \int_{\Omega}\left(\sum_{l=1}^{\infty} e^{-\lambda_{l} t} e_{l}(x) \overline{e_{l}(y)} \overline{e_{j}(x)} e_{k}(y) d y d x\right. \\
& =\sum_{l=1}^{\infty} e^{-\lambda_{l} t} \delta_{l j} \delta_{l k} \\
& =e^{-\lambda_{j} t} \delta_{j k} .
\end{aligned}
$$




\section{Corolário 5.29.}

$$
\int_{\Omega} k_{t}(x, x) d x=\sum_{j=1}^{\infty} e^{-\lambda_{j} t}
$$

Demonstração.

$$
\begin{aligned}
\int_{\Omega} k_{t}(x, x) d x & =\int_{\Omega} \sum_{j=1}^{\infty} e^{-\lambda_{j} t}\left|e_{j}(x)\right|^{2} d x \\
& =\sum_{j=1}^{\infty} e^{-\lambda_{j} t} \int_{\Omega}\left|e_{j}(x)\right|^{2} d x \\
& =\sum_{j=1}^{\infty} e^{-\lambda_{j} t},
\end{aligned}
$$

como desejado.

Enunciamos o Princípio do Máximo, que será utilizado no próximo resultado. Fisicamente, este princípio nos diz que, como o calor flui dos pontos mais quentes para os mais frios, então a temperatura máxima não pode ser atingida em um ponto interior, a não ser que $t=0$.

Proposição 5.30. (Princípio do Máximo)

Seja $\Omega \subset \mathbb{R}^{n}$ um domínio limitado e suave e $r>0$. Seja $f$ uma função suave em $(0, r) \times \Omega$, contínua em $[0, r] \times \bar{\Omega}$ e que satisfaz a equação do calor $f_{t}-\Delta f=0$ em $(0, r) \times \Omega$. Então temos

$$
\max _{(t, x) \in[0, r] \times \bar{\Omega}} f(t, x) \leq \max _{(t, x) \in(\{0\} \times \bar{\Omega}) \cup((0, r] \times \partial \Omega)} f(t, x) .
$$

O próximo resultado também é conhecido como o "princípio de não sentir a fronteira". A interpretação física dele é de que, para $t$ pequeno, o fluxo de calor em $\Omega$ é semelhante ao fluxo de calor em $\mathbb{R}^{n}$, pelo menos longe da fronteira $\partial \Omega$.

Lema 5.31. (Princípio de Kac)

Seja $\Omega \subset \mathbb{R}^{n}$ um domínio limitado e suave. Para todo $y \in \Omega$, definimos $t_{0}(y)=$ $\operatorname{dist}(y, \partial \Omega)^{2} / 2 n$. Então, para todo $x, y \in \Omega$, temos

$$
0 \leq k^{\mathbb{R}^{n}}(t, x, y)-k^{\Omega}(t, x, y) \leq\left\{\begin{array}{l}
(4 \pi t)^{-n / 2} e^{-\operatorname{dist}(y, \partial \Omega)^{2} / 4 t} \text { se } t \leq t_{0}(y) \\
\left(4 \pi t_{0}(y)\right)^{-n / 2} e^{-n / 2} \text { se } t>t_{0}(y)
\end{array}\right.
$$


Demonstração. A função $p$ definida por $p(t, x, y)=k^{\mathbb{R}^{n}}(t, x, y)-k^{\Omega}(t, x, y)$ é solução da equação do calor (Teorema 5.3.4 [2]). Assim podemos aplicar o Princípio do Máximo em $p(t, x, y)=k^{\mathbb{R}^{n}}(t, x, y)-k^{\Omega}(t, x, y)$ pensada como uma função de $t$ e $x$, onde os valores de $p$ na fronteira são dados por

$$
\begin{aligned}
& p(0, x, y)=0 \quad, x \in \bar{\Omega} \\
& p(t, x, y)=k^{\mathbb{R}^{n}}(t, x, y) \quad,(t, x) \in(0, \infty) \times \partial \Omega .
\end{aligned}
$$

Logo,

$$
p(t, x, y) \leq \sup _{(s, z) \in(0, t] \times \partial \Omega}(4 \pi s)^{-n / 2} e^{-|z-y|^{2} / 4 s} \leq \sup _{s \in(0, t]}(4 \pi s)^{-n / 2} e^{-\operatorname{dist}(y, \partial \Omega)^{2} / 4 s}
$$

A função $f(s)=(4 \pi s)^{-n / 2} e^{-\operatorname{dist}(y, \partial \Omega)^{2} / 4 s}$ atinge seu máximo em $t_{0}(y)$, é crescente para $s<t_{0}(y)$ e decrescente para $s>t_{0}(y)$.

\section{Proposição 5.32.}

Seja $\Omega \subset \mathbb{R}^{n}$ um domínio limitado e suave, então:

$$
\lim _{t \rightarrow 0^{+}} t^{n / 2} \int_{\Omega} k^{\Omega}(t, x, x) d x=|\Omega|(4 \pi)^{-n / 2}
$$

Demonstração.

O limite superior é consequência do Teorema 5.19 que, em particular, afirma que

$$
k^{\Omega}(t, x, x) \leq(4 \pi t)^{-n / 2}
$$

e portanto

$$
\limsup _{t \rightarrow 0^{+}} t^{n / 2} \int_{\Omega} k^{\Omega}(t, x, x) d x \leq|\Omega|(4 \pi)^{-n / 2} .
$$

Logo, basta mostrar que

$$
\liminf _{t \rightarrow 0^{+}} t^{n / 2} \int_{\Omega} k^{\Omega}(t, x, x) d x \geq|\Omega|(4 \pi)^{-n / 2} .
$$

Definimos, para $t>0$, os conjuntos

$$
\begin{aligned}
& \Omega_{1}(t)=\left\{x \in \Omega: \operatorname{dist}(x, \partial \Omega) \geq t^{1 / 4}\right\} \\
& \Omega_{2}(t)=\Omega \backslash \Omega_{1}(t) .
\end{aligned}
$$


Note que, quando $t \rightarrow 0,\left|\Omega_{2}(t)\right| \rightarrow 0$. Seja $t<(2 n)^{-2}$, então, para todo $x \in \Omega_{1}(t)$, temos

$$
t=t^{1 / 2} t^{1 / 2} \leq t^{1 / 2} /(2 n) \leq \operatorname{dist}(x, \partial \Omega)^{2} /(2 n)=t_{0}(x)
$$

e

$$
-\operatorname{dist}(x, \partial \Omega)^{2} /(4 t) \leq-t^{1 / 2} /(4 t)=-1 /(4 \sqrt{t})
$$

Portanto, pelo Princípio de Kac 5.31 temos

$$
k^{\mathbb{R}^{n}}(t, x, y)-k^{\Omega}(t, x, y) \leq(4 \pi t)^{-n / 2} e^{-1 /(4 \sqrt{t})} .
$$

Primeiro integramos sobre $\Omega_{1}(t)$, e fazendo $t \rightarrow 0^{+}$, temos

$$
t^{n / 2} \int_{\Omega_{1}(t)}\left(k^{\mathbb{R}^{n}}(t, x, x)-k^{\Omega}(t, x, x)\right) d x \leq|\Omega| e^{-1 /(4 \sqrt{t})}(4 \pi)^{-n / 2} \rightarrow 0
$$

Agora, integramos sobre $\Omega_{2}(t)$ e fazemos o limite $t \rightarrow 0^{+}$. Utilizamos a estimativa do núcleo Gaussiano (16) e o fato de que $k^{\Omega}(t, x, x) \geq 0$. Dessa forma temos

$$
\begin{aligned}
t^{n / 2} \int_{\Omega_{2}(t)}\left(k^{\mathbb{R}^{n}}(t, x, x)-k^{\Omega}(t, x, x)\right) d x & \leq t^{n / 2}\left|\Omega_{2}(t)\right|(4 \pi t)^{-n / 2} \\
& =\left|\Omega_{2}(t)\right|(4 \pi)^{-n / 2} \rightarrow 0 .
\end{aligned}
$$

Combinando as duas estimativas, temos

$$
\limsup _{t \rightarrow 0^{+}} t^{n / 2} \int_{\Omega}\left(k^{\mathbb{R}^{n}}(t, x, x)-k^{\Omega}(t, x, x)\right) d x=0 .
$$

Logo, como

$$
\lim _{t \rightarrow 0^{+}} t^{n / 2} \int_{\Omega} k^{\mathbb{R}^{n}}(t, x, x) d x=|\Omega|(4 \pi)^{-n / 2},
$$

concluímos que

$$
\liminf _{t \rightarrow 0^{+}} t^{n / 2} \int_{\Omega} k^{\Omega}(t, x, x) d x \geq|\Omega|(4 \pi)^{-n / 2}
$$

como esperado.

Juntando o Corolário 5.29 e a Proposição 5.32, temos o seguinte resultado. 


\section{Corolário 5.33.}

Seja $\Omega \subset \mathbb{R}^{n}$ um domínio limitado e suave, então:

$$
\lim _{t \rightarrow 0^{+}} t^{n / 2} \sum_{j=1}^{\infty} e^{-\lambda_{j} t}=\frac{|\Omega|}{(4 \pi)^{n / 2}}
$$

Finalmente, aplicando o Teorema de Karamata no resultado anterior, obtemos a Lei de Weyl.

Teorema 5.34. (Lei de Weyl)

Seja $\Omega \subset \mathbb{R}^{n}$ um domínio limitado e suave, $N(\lambda)$ o número de autovalores de Dirichlet para $\Omega$ (contando suas multiplicidades) menores ou iguais à $\lambda$ e $\omega_{n}=\frac{\pi^{n / 2}}{\Gamma(1+n / 2)}$ o volume da bola unitária em $\mathbb{R}^{n}$, então:

$$
\lim _{\lambda \rightarrow \infty} \frac{N(\lambda)}{\lambda^{n / 2}}=\frac{\omega_{n}}{(2 \pi)^{n}}|\Omega|
$$

Demonstração.

Segue do Teorema 5.21 e Corolário 5.33 que

$$
\lim _{\lambda \rightarrow \infty} \frac{N(\lambda)}{\lambda^{n / 2}}=\frac{|\Omega|}{\Gamma\left(\frac{n}{2}+1\right)(4 \pi)^{n / 2}}=\frac{\omega_{n}}{(2 \pi)^{n}}|\Omega|
$$

concluindo a demonstração. 


\section{Considerações finais}

A Lei de Weyl nos diz que podemos escutar a área de um tambor. Dizemos, então, que a área é uma invariante espectral. De fato, conhecendo o espectro do Laplaciano podemos determinar a área de seu domínio. Após o artigo de Kac de 1966 [28], o seguinte debate foi promovido: quais outras características geométricas são invariantes espectrais? Nesta seção, enunciamos alguns resultados posteriores a Lei de Weyl que ampliam o conhecimento em torno desta discussão.

Para facilitar a exposição, seguem duas definições úteis. Dizemos que dois conjuntos abertos e limitados são isoespectrais se os autovalores do operador Laplaciano com condições de contorno de Dirichlet em cada um desses conjuntos são iguais. Dois conjuntos são congruentes se forem invariantes por transformações rígidas, ou seja, $\Omega_{1}$ e $\Omega_{2} \subset \mathbb{R}^{n}$ são congruentes se existe uma matriz ortogonal $B$ e um vetor $b$ em $\mathbb{R}^{n}$ tal que $\Omega_{1}=\left\{B x+b: x \in \Omega_{2}\right\}$. É claro que dois conjuntos congruentes são isoespectrais, já que o Laplaciano é invariante por transformações rígidas.

Antes do artigo de Kac, em 1954, Pleijel [36] mostrou que o perímetro também é uma invariante espectral para domínios planos. Se $A$ é a área de um domínio plano e $P$ o seu perímetro, então

$$
\sum_{m=1}^{\infty} e^{-\lambda_{m} t}=\frac{A}{2 \pi t}-\frac{P}{4 \sqrt{2 \pi t}}, \text { quando } t \rightarrow 0 .
$$

Em seu artigo, Kac [28] reformulou este resultado em termos do traço do núcleo do calor e mostrou que conseguimos escutar se um tambor é circular ou não, como consequência da clássica desigualdade isoperimétrica que afirma que

$$
P^{2} \geq 4 \pi A
$$

com a igualdade ocorrendo apenas para círculos. Kac também conjecturou que, se o domínio tiver $b$ buracos, então a fórmula seria

$$
\sum_{m=1}^{\infty} e^{-\lambda_{m} t}=\frac{A}{2 \pi t}-\frac{P}{4 \sqrt{2 \pi t}}+\frac{1-b}{6}, \text { quando } t \rightarrow 0
$$

Este resultado foi provado no ano seguinte por Mckean e Singer [31]. Na verdade, eles mostraram que a característica de Euler é uma invariante espectral e consequentemente podemos escutar o número de buracos de um tambor. 
Vimos na introdução que em 1992 Carolyn Gordon e David Webb [22] apresentaram duas regiões diferentes porém isoespectrais, respondendo negativamente à questão inicial formulada por Kac, isto é, o espectro do Laplaciano não determina completamente a geometria de seu domínio. Entretanto, a discussão prosseguiu com a busca de novas relações entre o espectro do Laplaciano e as características geométricas do seu domínio.

Durso demonstrou em 1988 [14] que se dois domínios triangulares são isoespectrais, então eles são congruentes. Em 2010, Hezari e Zelditch [27] fizeram progresso mostrando o que parece ser a primeira resposta positiva em dimensões maiores. Eles mostraram que se dois domínios limitados, analíticos e que satisfazem certas condições de simetria são isospectrais, então eles são congruentes. Já em 2015, Lu e Rowlett [30] mostraram o curioso fato de que podemos escutar se um tambor possui cantos ou, em outras palavras, que a falta de suavidade da fronteira do domínio é uma invariante espectral.

Essas são algumas das características geométricas que os matemáticos mostraram serem invariantes espectrais. Muito ainda não se sabe e novas conexões entre o espectro do Laplaciano e a geometria de seu domínio aguardam para ser descobertas. Em particular, os contraexemplos encontrados até agora de domínios não-congruentes e isoespectrais são todos com fronteira não suave. Isso nos leva à pergunta ainda sem resposta: domínios suaves isoespectrais são congruentes? 


\section{Referências Bibliográficas}

[1] Helmut Abels. Pseudodifferential and singular integral operators: an introduction with applications. Walter de Gruyter, 2011.

[2] Wolfgang Arendt. Heat kernels. In Lecture Notes of the TULKA internet seminar, 2005.

[3] Wolfgang Arendt. Vector-valued Laplace Transforms and cauchy problems. Birkhäuser, Basel, 2011.

[4] Wolfgang Arendt and Alexander V Bukhvalov. Integral representations of resolvents and semigroups. In Forum Mathematicum, volume 6, pages 111-136. Walter de Gruyter, Berlin/New York, 1994.

[5] Wolfgang Arendt, Ralph Chill, Christian Seifert, Hendrik Vogt, and Jürgen Voigt. Form methods for evolution equations, and applications. In 18th Interent Seminar on evolution equations, Lecture Notes. http://www. mat. tuhh. de/isem18, 2014.

[6] Wolfgang Arendt, Robin Nittka, Wolfgang Peter, and Frank Steiner. Weyl's law: spectral properties of the laplacian in mathematics and physics. Mathematical analysis of evolution, information, and complexity, pages 1-71, 2009.

[7] Geraldo Botelho, Daniel Pellegrino, and Eduardo Teixeira. Fundamentos de Análise Funcional. SBM, 2015.

[8] Thierry Cazenave and Alain Haraux. An introduction to semilinear evolution equations, volume 13. Oxford University Press on Demand, 1998.

[9] Pei-Kun Chang and Dennis DeTurck. On hearing the shape of a triangle. Proceedings of the American Mathematical Society, 105(4):1033-1038, 1989.

[10] Barry Cipra. You can't hear the shape of a drum. Science, 255(5052):1642-1644, 1992.

[11] Kiril Datchev and Hamid Hezari. Inverse problems in spectral geometry. Inverse problems and applications: Inside Out II, 60:455-486, 2011.

[12] César R De Oliveira. Introdução à análise funcional. Impa, 2001. 
[13] Jozef Dodziuk. Eigenvalues of the laplacian and the heat equation. The American Mathematical Monthly, 88(9):686-695, 1981.

[14] Catherine Durso. On the inverse spectral problem for polygonal domains. $\mathrm{PhD}$ thesis, Massachusetts Institute of Technology, 1988.

[15] Klaus-Jochen Engel and Rainer Nagel. One-parameter semigroups for linear evolution equations. In Semigroup Forum, volume 63, pages 278-280. Springer, 2001.

[16] Klaus-Jochen Engel and Rainer Nagel. A short course on operator semigroups. Springer Science \& Business Media, 2006.

[17] Lawrence Evans. Partial differential equations. American Mathematical Society, Providence, R.I, 2010.

[18] Gerald B Folland. Introduction to partial differential equations. Princeton university press, 1995.

[19] Gerald B. Folland. Fourier Analysis and Its Applications (Pure and Applied Undergraduate Texts). American Mathematical Society, 2009.

[20] Gerald B Folland. Real analysis: modern techniques and their applications. John Wiley \& Sons, 2013.

[21] Carolyn Gordon and David Webb. You can't hear the shape of a drum. American Scientist, 84(1):46-55, 1996.

[22] Carolyn Gordon, David L Webb, and Scott Wolpert. One cannot hear the shape of a drum. Bulletin of the American Mathematical Society, 27(1):134-138, 1992.

[23] HPW Gottlieb. Hearing the shape of an annular drum. The ANZIAM Journal, 24(4):435-438, 1983.

[24] Gerd Grubb. Distributions and operators, volume 252. Springer Science \& Business Media, 2008.

[25] Markus Haase. Lectures on functional calculus. In 21st International Internet Seminar, 2018. 
[26] Richard Haberman. Elementary applied partial differential equations, volume 987. Prentice Hall Englewood Cliffs, NJ, 1983.

[27] Hamid Hezari and Steve Zelditch. Inverse spectral problem for analytic $(\mathbb{Z} / 2 \mathbb{Z})^{n}$ symmetric domains in $\mathbb{R}^{n}$. Geometric and Functional Analysis, 20(1):160-191, 2010.

[28] Mark Kac. Can one hear the shape of a drum? The american mathematical monthly, 73(4P2):1-23, 1966.

[29] S Kesavan. Listening to the shape of a drum. Resonance, 3(10):49-58, 1998.

[30] Zhiqin Lu and Julie M Rowlett. One can hear the corners of a drum. Bulletin of the London Mathematical Society, 48(1):85-93, 2015.

[31] Henry P McKean, Isadore M Singer, et al. Curvature and the eigenvalues of the laplacian. J. Differential Geometry, 1(1):43-69, 1967.

[32] Liviu I Nicolaescu. Topics in topology. spring 2010. pseudo-differential operators and some of their geometric applications1. 2010.

[33] Alberto Parmeggiani. Spectral theory of non-commutative harmonic oscillators: an introduction. Springer, 2010.

[34] Amnon Pazy. Semigroups of linear operators and applications to partial differential equations, volume 44. Springer Science \& Business Media, 2012.

[35] Gert K Pedersen. Analysis now, volume 118. Springer Science \& Business Media, 2012.

[36] Åke Pleijel. A study of certain green's functions with applications in the theory of vibrating membranes. Arkiv för Matematik, 2(6):553-569, 1954.

[37] MH Protter. Can one hear the shape of a drum? revisited. Siam Review, 29(2):185$197,1987$.

[38] Michael Reed. Methods of modern mathematical physics: Functional analysis. Elsevier, 2012.

[39] Michael Reed, Barry Simon, Barry Simon, and Barry Simon. Methods of modern mathematical physics, volume 1. Elsevier, 1972. 
[40] Michael Renardy and Robert C Rogers. An introduction to partial differential equations, volume 13. Springer Science \& Business Media, 2006.

[41] John Roe. Elliptic operators, topology, and asymptotic methods. Chapman and Hall/CRC, 2013.

[42] Walter Rudin. Functional analysis. McGraw-Hill, New York, 1991.

[43] Konrad Schmüdgen. Unbounded self-adjoint operators on Hilbert space, volume 265. Springer Science \& Business Media, 2012.

[44] Laurent Schwartz and Institut de mathématique (Strasbourg). Théorie des distributions, volume 2. Hermann Paris, 1957.

[45] Walter A Strauss. Partial differential equations: An introduction. Wiley, 2007.

[46] Robert S Strichartz. A guide to distribution theory and Fourier transforms. World Scientific Publishing Company, 2003.

[47] Washington University Photographic Services. David webb and carolyn gordon, former faculty at washington university in st. louis, with paper models of a pair of 'sound-alike' drums. [Online; accessed 14-May-2019].

[48] Hermann Weyl. Über die asymptotische verteilung der eigenwerte. Nachrichten von der Gesellschaft der Wissenschaften zu Göttingen, Mathematisch-Physikalische Klasse, 1911:110-117, 1911.

[49] Wikipedia contributors. Bessel functions of the first kind, $j_{0}(x)$ in red, $j_{1}(x)$ in green and $j_{2}(x)$ in blue., 2008. [Online; accessed 14-May-2019].

[50] Wikipedia contributors. Illustration of vibrations of a drum., 2008. [Online; accessed 14-May-2019].

[51] Wikipedia contributors. Illustration of vibrations of a drum., 2008. [Online; accessed 14-May-2019].

[52] Wikipedia contributors. Illustration of vibrations of a drum., 2008. [Online; accessed 14-May-2019]. 
[53] Man Wah Wong. An introduction to pseudo-differential operators, volume 6. World Scientific Publishing Company, 2014. 